ANTI-FOG COATINGS USING

THE SUPER-HYDROPHOBIC

APPROACH

A Thesis

presented to

the Faculty of the Graduate School

University of Missouri-Columbia

In Partial Fulfillment

of the requirements for the Degree

Master of Science

by

RIBERET ALMEIDA

Dr. Shubhra Gangopadhyay, Thesis Supervisor

AUGUST, 2008 
The undersigned, appointed by the Dean of the Graduate School, have examined the thesis entitled

\section{ANTI-FOG COATINGS USING THE SUPER-HYDROPHOBIC APPROACH}

Presented by Riberet Almeida

A candidate for the degree of Master of Science

And hereby certify that in their opinion it is worthy of acceptance.

\begin{tabular}{l}
\hline Dr. Shubhra Gangopadhyay \\
\hline Dr. Maruf Hossain \\
\hline
\end{tabular}

Dr. Luis Polo-Parada 


\section{ACKNOWLEDGEMENTS}

I am greatly indebted to my advisor Dr. Shubhra Gangopadhyay \& also to Dr. Keshab Gangopadhyay for accepting me into their research group and for mentoring me throughout this thesis.

I would also like to thank my thesis committee members Dr. Maruf Hossain and Dr. Luis Polo-Parada, for all their helpful discussions in fabricating this Super-hydrophobic surface.

Among the other people, I would like to thank Dr. Uma Sampathkumaran from Innosense LLC and Dr. Venumadhav Korampally for all their helpful discussions and ideas and Mr. Lou Ross from the EMC facility at UMC for his time in obtaining the SEM images.

I would also like to specially thank my parents and my girlfriend Theresa for all their financial and moral support all through this while.

Financial support was provided by the National Science Foundation STTR for "Low-cost processing of nanoporous, superhydrophilic, multifunctional coatings for glass and plastic surfaces"

-Riberet Almeida 


\section{TABLE OF CONTENTS}

ACKNOWLEDGEMENTS .................................... ii

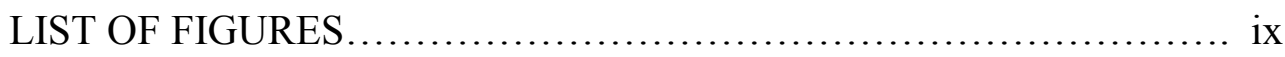

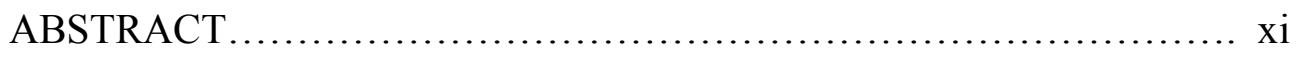

CHAPTER INTRODUCTION....................................... 1

I

1.1 INTRODUCTION............................... 1

1.2 SUPER-HYDROPHOBIC SURFACES THAT EXIST IN

NATURE........................................ 2

1.2.1 Plant Species: the Sacred Lotus Leaf.............. 2

1.2.2 Animal Species: The water strider ................ 3

1.3 THESIS

MOTIVATION.......................................... 3

1.4 THESIS OUTLINE................................. 4

CHAPTER LITERATURE REVIEW .......................... 6

II

2.1 TECHNIQUES USED IN THE DEVELOPMENT OF SUPER-HYDROPHOBIC SURFACES .................. 6

2.1.1 Top-Up and Bottom-Down Approaches............... 6

2.1.2 Templation.................................. 6

2.1.3 Plasma treatment of surfaces..................... 7

2.1.4 Colloidal Assemblies................................ 8

2.1.5 Layer by Layer deposition(LBL) .................. 8

2.1.6 Sol-gel methods.............................. 10 
CHAPTER SUPER-HYDROPHOBIC SURFACES

III

3.1 INTRODUCTION................................... 13

3.1.1 Super-hydrophobic Surfaces....................... 14

3.1.2 Wenzel's Theory................................. 14

3.1.3 Cassie-Baxter Theory ............................ 16

3.2 SURFACE ENERGIES................................. 17

3.3 THE SOL-GEL CHEMISTRY ............................ 19

3.3.1 The Theory ..................................... 19

3.3.2 Templating using Organic Materials................ 20

3.4 SILANE COUPLING AGENTS......................... 21

3.4.1 The Silane Bond to the Inorganic Substrate.......... 21

3.5 SURFACE CHARACTERIZATIONS.................... 23

3.5.1 Contact Angle measurement system............... 23

3.5.2 Scanning Electron Microscope........................ 24

3.5.3 Atomic Force Microscopy......................... 24

CHAPTER SUPER-HYDROPHOBIC SURFACES: FABRICATION 25

IV

4.1 POROUS SILICA SOL-GEL EXPERIMENTAL PROCEDURE.................................... 25

4.1.1 Preparation of Sol-gel Films....................... 26

4.1.2 Low Temperature processing of films.............. 27

4.2 NANO-MATERIALS SYNTHESIZED ................. 28

4.2.1 Preparation of Organo-silicate(PMSSQ) Nano-rods. 28

4.2.2 Preparation of PMSSQ Nano-particles.............. 29

4.3 LOADING SOL-GEL FILMS WITH ORGANO-

SILICATE NANO-RODS ............................ 30

4.3.1 Experimental procedure........................ 30

4.3.2 Experimental procedure for Sol-gel synthesis........ 30

4.4 TEFLON THIN FILMS ................................ 32

4.4.1 PTFE(Poly Tetra Fluoro Ethylene)................. 32

4.4.2 Porous polymer films using micro-emulsions........ 33

4.4.3 The Micro-emulsion Process: Experimental procedure..................................... 33 
4.5 Teflon AF 1600 films embedded with SiC Nano-particles... 33

4.5.1 Experimental procedure............................ 34

4.5.2 Experiment with the Silicon-Oxy-Carbide Intermediate Layer............................... 36

4.5.3 Experiment with the $0.125 \%(\mathrm{wt} \%)$ Teflon Configuration.................................... 36

4.6 Hardness Measurements.................................. 37

CHAPTER RESULTS AND DISCUSSION.......................... 38 $\mathrm{V}$

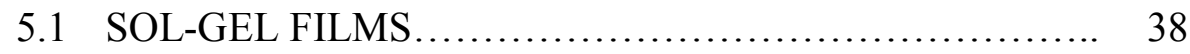

5.1.1 The effect of Oxygen Plasma........................ 38

5.2 CHARACTERISATION OF LOW TEMPERATURE

PROCESSED FILMS..................................... 41

5.2.1 NO Annealing..................................... 42

5.2 .2 12Hr Annealing.................................... 43

$5.2 .324 \mathrm{Hr}$ Annealing............................... 43

5.3 OXYGEN PLASMA TREATED SAMPLES(THE SAME ABOVE SAMPLES) .................................. 44

5.3.1 -NO Annealing, 1min Oxy Plasma treated samples.... 44

5.3.2 -12 Hr Annealing 1min Oxy Plasma treated samples... 45

5.3.3 -24Hr Annealing 1min Oxy Plasma treated s amples.. 46

5.4 FTIR CHARACTERIZATIONS

5.4.1 FTIR plots before Oxygen plasma treatment/water immersion and CTAB removal of low temperature processed films

5.4.2 FTIR plots after Oxygen plasma treatment/water immersion but before CTAB removal............... 48

5.4.3 FTIR plots after CTAB removal WITHOUT Oxygen Plasma(By only exposing it to Ethanol vapours).

5.4.4 FTIR plots after CTAB removal WITHOUT Oxygen Plasma (By exposing it to Ethanol vapors + washing with cold Ethanol).

5.5 DISCUSSION ON THE EFFECT OF MILD OXYGEN PLASMA.............................................. 51

5.6 HYDROPHOBIC APPROACH: NANO-MATERIALS SYNTHESIS. 
5.6.1 Organo-Silicate Nanorods........................ 54

5.6.2 Non-porous Silica sol-gel films: Loading with organo-silicate Nano-rods....................... 56

5.6.3 Discussion on nano-rod loaded sol-gel films.......... 58

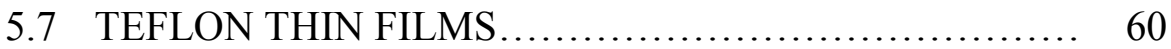

5.7.1 Porous polymer films using micro-emulsions........ 60

5.7.2 Transmission Spectra........................... 63

5.7.3 Porous Teflon using PPG \& PGMEA.............. 65

5.7.4 After removing excess PGMEA from solution and only keeping the milky solution..................... 67

5.7.5 Contact Angles for the above substrates............ 67

5.7.6 CA Images of films using the Teflon:PGMEA:PPG $=3: 1.5: 3$ ratios............................... 68

5.7.7 CA Images of films using the Teflon:PGMEA:PPG $=3: 3: 1.5$ ratios............................... 69

5.7.8 Discussion on porous Teflon films using microemulsions

5.8 RESULTS: TEFLON AF 1600 FILMS EMBEDDED WITH SIC NANO-PARTICLES.

5.8.1 2\% Teflon Films embedded with $0.1 \%(w t \%)$ SiC Nano-particles

5.9 EXPERIMENT WITH DIFFERENT WT\% SIC NANOPARTICLES IN 0.5\%(WT\%) TEFLON SOLUTIONS..... 75 5.9.1 UV-VIS transmission Spectroscopy on above films... 75

5.10 EXPERIMENT WITH $\quad 0.1 \%(\mathrm{WT} \%) \quad$ SIC NANOPARTICLES IN 0.125\%(WT\%) TEFLON SOLUTION ... 77 5.10.1 Loading with $0.1 \%(\mathrm{wt} \%)$ SiC Nano-particles........ 77

$\begin{array}{llccr}5.11 & \text { EXPERIMENT } & \text { WITH } & 0.1 \%(\mathrm{WT} \%) \mathrm{SIC} & \text { NANO- } \\ & \text { PARTICLES } & \text { IN } & 0.125 \%(\mathrm{WT} \%) & \text { TEFLON }\end{array}$ SOLUTION(TRIPLE COAT) ....................... 78 
5.12 EXPERIMENT WITH DIFFERENT WT\% OF SIC NANO-PARTICLES IN 0.125\%(WT\%) TEFLON

SOLUTION ..................................................... 80

5.12.1 Loading with $0.25 \%(\mathrm{wt} \%) \mathrm{SiC}$ - Single layer....... 80

5.12.2 Loading 0.5\%(wt $\%) \mathrm{SiC}$ - Single layer.............. 81

5.12 .3 Loading with $0.5 \%(\mathrm{wt} \%) \mathrm{SiC}$ - Triple layered....... 82

5.12.4 Transmission Spectra of the above[0.5\%(wt\%) SiC loaded in $0.125 \%$ (wt $\%)$ Teflon solution(dual coat)]

Super-hydrophobic films.

5.13 EXPERIMENT WITH 0.0625\%(WT\%) TEFLON

SOLUTION.

84

5.13.1 $0.5 \%(\mathrm{wt} \%) \mathrm{SiC}$ - Single layer.

5.13.2 Transmission Spectra of $[0.5 \%(\mathrm{wt} \%) \mathrm{SiC}$ embedded in $0.0625 \%(w t \%)$ Teflon solution] Superhydrophobic films.

5.14 EXPERIMENT WITH DUAL LAYER WITH DIFFERENT COMPOSITIONS.

$5.14 .1[0.5 \%(\mathrm{wt} \%) \mathrm{SiC}$ in $0.0625 \%$ Teflon $]+[0.1 \%(\mathrm{wt} \%)$

$\mathrm{SiC}$ in $0.0625 \%$ Teflon ] (double layer)...............

$5.14 .2[0.5 \%(\mathrm{wt} \%) \mathrm{SiC}$ in $0.0625 \%$ Teflon] $+[0.1 \%(\mathrm{wt} \%)$ $\mathrm{SiC}$ in $0.0625 \%$ Teflon $]+[0.1 \%(\mathrm{wt} \%) \mathrm{SiC}$ in $0.0625 \%$ Teflon ] (triple layer).

5.14.3 Transmission Spectra of the above Superhydrophobic films

5.14.4 SEM characterization of above dual $([0.5 \%(\mathrm{wt} \%)$ $\mathrm{SiC}$ in $0.0625 \%$ Teflon $]+[0.1 \%(\mathrm{wt} \%) \mathrm{SiC}$ in $0.0625 \%$ Teflon] coated film).

5.15 EXPERIMENT WITH SILICON-OXY CARBIDE INTERMEDIATE LAYER.

5.16 HARDNESS MEASUREMENTS ........................ 91

5.16.1 Discussion on nano-particle loaded Teflon films.

5.17 DISCUSSION ON 2\%(WT\%) TEFLON FILMS LOADED WITH $0.1 \%($ WT $\%)$ SIC. 
5.18 CALCULATION OF SOLID-AIR AREA FRACTIONS,

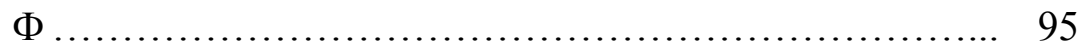

5.18.1 Discussion on calculation of Solid-Air area fraction 95

5.18.2 Apparent Contact Angles...................... 96

5.19 MEASUREMENT OF SURFACE ENERGIES............ 100

5.20 CALCULATION OF WENZEL ROUGHNESS FACTOR 'R' AND RMS ROUGHNESS......................... 101

$5.20 .10 .1 \%(\mathrm{wt} \%) \mathrm{SiC}$ nano-particles embedded in 0.125\%(wt \%) Teflon: Single Layer configuration..................................... 102

$5.20 .20 .1 \%(\mathrm{wt} \%) \mathrm{SiC}$ nano-particles embedded in $0.125 \%(w t \%)$ Teflon: 3 Layer configuration......... 103

$5.20 .30 .5 \%(\mathrm{wt} \%) \mathrm{SiC}$ nano-particles embedded in $0.125 \%(w t \%)$ Teflon: Single Layer configuration... 104 $5.20 .40 .5 \%(\mathrm{wt} \%) \mathrm{SiC}$ nano-particles embedded in $0.125 \%(\mathrm{wt} \%)$ Teflon: 3 Layer configuration......... 105

$5.20 .50 .5 \%(\mathrm{wt} \%) \mathrm{SiC}$ nano-particles embedded in 0.125\%(wt\%) Teflon: 10 Layer configuration....... 106

5.21 DISCUSSION ON RMS ROUGHNESS................ 107

CHAPTER CONCLUSIONS ....................................... 111

VI

6.1 RESULTS SUMMARY .............................. 111

6.2 FUTURE WORK..................................... 112

REFERENCES ................................................... 114 


\section{LIST OF FIGURES}

Figure 5-1 SEM depicting the structural changes that are brought about in lowtemperature processed sol-gel films when exposed to Oxygen Plasma.

Figure 5-2 FTIR plots before Oxygen plasma treatment/water immersion and CTAB

removal of low temperature processed films.

Figure 5-3 FTIR plots after Oxygen plasma treatment/water immersion but before CTAB removal.

Figure 5-4 FTIR plots after CTAB removal WITHOUT Oxygen Plasma(By only exposing it to Ethanol vapors).

Figure 5-5 FTIR plots after CTAB removal WITHOUT Oxygen Plasma (By exposing it to Ethanol vapors + washing with cold Ethanol).

Figure 5-6 SEM depicting the collapse of the upper layers of the low temperature processed films due to Oxygen Plasma. 52

Figure 5-7 PMSSQ(poly(methylsilsesquioxane)) Nano-rods 55

Figure 5-8: Image of Teflon micro-emulsion films using Ethanol as the non-solvent. .... 60

Figure 5-9 UV-VIS transmission spectra of 3\% ethanol based micro-emulsion films ..... 63

Figure 5-10 UV-VIS transmission spectra of 5\% ethanol based micro-emulsion films ... 64

Figure 5-11 UV-VIS transmission spectra of 10\% ethanol based micro-emulsion films. 64

Figure 5-12 SiC particles can be seen embedded in the Teflon films in the images above(1) .72

Figure 5-13 SiC particles can be seen embedded in the Teflon films in the images above(2) 72

Figure 5-14 One section of the substrate where the Teflon layer has been detached from the substrate and been suspended off the substrate.

Figure 5-15 One section of the substrate where the Teflon layer did not adhere well enough to the substrate due to absence of adhesion promoter and the $\mathrm{SiC}$ nano-particles

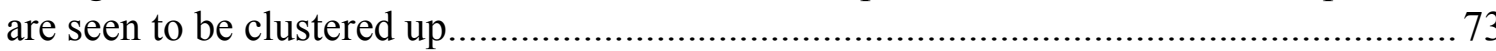

Figure 5-16 UV-VIS spectroscopy of the SiC embedded Teflon films. ........................ 76

Figure 5-17 UV-VIS spectroscopy of dual coated $0.5 \%$ films. 83 
Figure 5-18 UV-VIS spectroscopy of Single Layer $0.5 \% \mathrm{SiC}$ in $0.0625 \%$ Teflon. 85

Figure 5-19 UV-VIS spectroscopy of dual Layer $0.5 \% \mathrm{SiC}+0.1 \% \mathrm{SiC}$ in $0.0625 \%$ Teflon

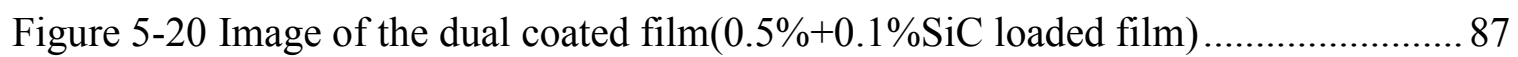

Figure 5-21 SEM images of above dual coated Superhydrophobic films(1)................... 88

Figure 5-22 SEM images of above dual coated Superhydrophobic films(2) .................. 88

Figure 5-23 SEM and corresponding Backscattered images of SiCO/Teflon films .........89

Figure 5-24 Diagram of model presented by Rob J. Klein et al [27] ............................. 98

Figure 5-25 Graph of Surface energies of different surfaces ..................................... 100

Figure 5-26 Graph of RMS Roughness v/s Solid-Air Area fraction ' $\Phi$ ' ....................... 107

Figure 5-27 Graph of the Wenzel and modified Cassie-Baxter theoretical curves with

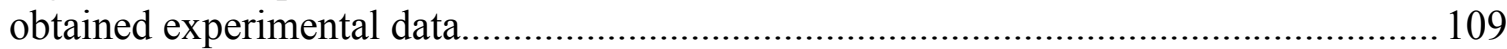




\title{
ANTI-FOG COATINGS USING THE SUPER-HYDROPHOBIC APPROACH
}

\author{
Riberet Almeida \\ Dr. Shubhra Gangopadhyay, Thesis Supervisor \\ ABSTRACT
}

Surfaces with water contact angles in excess of $150^{\circ}$ have been attracting a great deal of attention. The work done here was in the design and development of an anti-fog coating. The hydrophilic as well as the hydrophobic approaches were investigated before developing the super-hydrophobic coatings. Accordingly, various phenomenon such as the adherence of snow or raindrops and friction drag are expected to be inhibited or reduced on such surfaces.

Hydrophobic properties are enhanced by increasing surface roughness. Superhydrophobic surfaces require both appropriate surface roughness and low surface energy materials and numerous methods to attain these requirements have been demonstrated. We have made extensive use of nano-particles to help us in achieving the roughness that was needed to create these super-hydrophobic surfaces. Also low surface energy materials such as PTFE and THV were investigated as potential candidates for the matrix materials in which the particles could be embedded. The films created in our labs were characterized extensively using SEM(Scanning Electron Microscopy), Water Contact angles, Spectroscopic Ellipsometry, AFM(Atomic Force Microscopy) and UV-VIS Spectroscopy. 


\section{CHAPTER I: INTRODUCTION}

\subsection{Introduction}

There has been a great deal of interest in developing super-hydrophobic surfaces in the last few years. The driving force behind the creation of these surfaces is the ability to mimic super-hydrophobic surfaces in nature. Many plant and animal species in nature have to ability to remain clean or simply possess self cleaning properties.

The self-cleaning effect is evident from the lotus leaf. The underlying mechanism of such a surface has been thoroughly studied. When a water droplet moves down a tilted superhydrophobic surface, due to the high contact angle, effective macroscopic slip occurs on scales consistent with the characteristic size of the surface features. This causes a water droplet to behave like an elastic ball rather than a fluid. In case of normal hydrophobic surfaces, the non-slip condition causes any water droplets falling across any dirt particles in its path to displace them towards the side of the droplet and redeposit them behind the droplet. In case of super-hydrophobic or water repellant rough surfaces, the solid-water interface is minimized. Hence water droplets tend to take on more circular shapes and carry away with it any contaminants present in its path. This phenomenon is dubbed as the "lotus effect"

Interestingly the enhancement of the contact angle with roughness was pointed out 60 years earlier before the discovery of the self-cleaning mechanism of the lotus leaf[8]. No one could associate this phenomenon to the self cleaning mechanism of the lotus leaf. The observation of hydrophobicity being associated to the topology of the surface of the lotus leaf was reported by Barthlott and Neinhuis[10]. 
Super-hydrophobic surfaces have a great deal of industrial and practical applications. Some of the current applications are anti-fog coatings which is the focus of this thesis, self cleaning skyscraper windows, prevention of adhesion of snow to antennas, self cleaning traffic indicators, the reduction of frictional drag on ship hulls, stain resistant textiles[2], anti-oxidation[3], minimization of contamination in biological applications, and lowering the flow resistance in micro-fluidic devices[4].

\subsection{Super-hydrophobic surfaces that exist in Nature}

\subsubsection{Plant Species: the Sacred Lotus Leaf}

There are many plant and animal species in nature that exhibit water repellant surfaces. In case of plant surfaces, it has been found out that epicuticular wax crystalloids that cover the cuticular surface in a regular micro-relief of about 1-5um in height[5][6] responsible for water repellency of such surfaces. The sacred lotus plant is one such example which exhibits water contact angles of $161^{\circ}$ with a hysteresis of $2^{\circ}$. On close examination of the surface of a lotus leaf with SEM imaging, it was found that the surface was composed of a hierarchical micro and nano-structure morphology[7], one at $10 \mathrm{um}$ and one at $100 \mathrm{~nm}$. Now in case of the lotus leaf, the epicuticular wax that's secreted by this plant has a water contact angle of $110^{\circ}$. The roughness at these two levels along with the low surface energy wax gives these surfaces their super-hydrophobic characteristics. The "lotus effect" comes from the fact that the water drops rolling off these surfaces carry with them any contaminants that are present on these surfaces.

The Indian Cress is a similar plant which has a surface morphology similar to that of the lotus leaf[1] but has surface roughness in three different length scales of micrometer length scales. The first length scale is few tens of microns by the epidermal cells, the 
second being in the lum scale due to the wax crystals on the top of the cell surface and the third being the distance between the wax crystal bundles $\sim 5 \mathrm{um}$. The other plant is the Lady's Mantle which has a hairy water repellant surface. These plant surfaces were studies in detail by Otten and Herminghaus[1].

\subsubsection{Animal Species: The water strider}

The water strider is one such insect that can glide on water or walk on the surface of moving water. This is possible because of the numerous oriented needle shaped setae on the legs[22][9]. These structures are needle shaped with diameters ranging from $3 \mathrm{um}$ down to a couple of hundred nanometers. These setae are 50um in length and oriented at an inclination angle of 20degrees from the surface of each leg. The force that each leg can sustain is about 15 times the total body weight before piercing the water surface. This phenomenon is ascribed to the hierarchical structure of the legs.

Due to the immense number of application in creating super-hydrophobic coatings on a wide variety of surfaces, a lot of research has been carried out in order to easily manufacture or produce such surfaces.

\subsection{Thesis Motivation}

The main aim of this thesis was focused on the development of super- hydrophobic surfaces that could have use in anti-fog applications. For anti-fog application, the developed coating would need to posses very high transparencies in the visible region and at the same time have good adhesion and durability. For anti-fog application, a study on the behavior of water droplets due to moisture condensation, on such surfaces is very crucial. The water droplet interaction with the surface from a macroscopic view is 
different from the microscopic viewpoint especially when the droplet size is comparable to the surface feature sizes. Developing super-hydrophobic surfaces wherein microscopic water droplets formed due to moisture condensation, could grow and easily roll-off due to the high contact angles was something yet to be explored. Even the lotus leaf acts like a ‘sticky' surface when water is condensed forming microscopic droplets which are trapped between the nano-hairy regions on the leaf surface[21]. This happens because of the hydrophilic nature of the waxy material covering the leaf surface which can be made hydrophobic by surface texturing. It would be very interesting to observe the anti-fogging properties of a surface with surface morphology similar to a lotus leaf and having a very low surface energy.

\subsection{Thesis outline}

In the last three years a lot of new approaches have been investigated in creating such super-hydrophilic surface which have been spoken in great detail in chapter 2. Chapter 2 also contains literature on the development of super-hydrophobic surfaces via the addition of nano-particle fillers like silica nano-particles[11][12] or Silicon Carbide nano-

particles, templating[13][14], micro-machining, controlled crystallization, lithography[17][18], chemical vapor deposition, phase separation, and plasma etching[15][16].

Chapter 3 describes the theory and the fundamentals in the design of superhydrophobic surfaces, as the work described in this thesis, has been on the development of such surfaces. Chapter 3 also talks about the theory behind the wetting of surfaces, the classification and characterization of super-hydrophobic 
surfaces and the different models used to characterize super-hydrophobic surfaces. The Sol-gel theory has also been presented in this chapter.

Chapter 4 contains the experimental section in the fabrication of such super-hydrophobic surfaces. The different approaches that were used in the fabrication of such surfaces have been presented in chapter 4 along with their respective experimental procedures. This chapter also contains the experimental section for the nano-materials that were synthesized and which were used as building blocks in the fabrication of highly water repellant surfaces.

Chapter 5 contains the results of all the fabrication procedures from the experimental section in chapter 4 including the characterization data of such surfaces in the form of contact angle measurements, spectroscopic ellipsometry, optical images, AFM(Atomic force microscopy) images, SEM(Scanning electron microscopy) images, TEM(transmission electron microscopy) images, FTIR(Fourier transform infrared spectroscopy), UV-VIS spectroscopy. It also contains the hardness data of some of the films using AFM nano-indentation. A discussion on the results in presented in this chapter and some of the data on surface area fractions were used with existing superhydrophobic models to get an estimate of the predicted contact angles which were compared with the actual contact angles.

In chapter 6 , general conclusions are drawn and future work suggested. 


\section{CHAPTER II: LITERATURE REVIEW}

\subsection{Techniques used in the development of Super-hydrophobic Surfaces}

\subsubsection{Top-Up and Bottom-Down Approaches}

Surfaces can either be built from the top to bottom or from the bottom to the top and are known as Top-Down and Bottom-Up Approaches. Templating of Surfaces including lithography and the plasma polymerization of surfaces come under the Top-Down approaches while the techniques such as Colloidal assemblies, layer by layer depositions and chemical depositions encompass bottom up approaches. These approaches include self-organization and Self-Assembly.

2.1.2 Templation: is the method in which a surface with the desired feature or surface morphologies is taken and replicated using molding, followed by a lift-off of the replica or the dissolution of the template[22]. Polymeric super-hydrophobic surfaces often use templating in their creation. Naturally occurring materials in nature such as Lotus leaves and other super-hydrophobic inorganic surfaces can be used as the templates for the creation of such artificial replicas. The most commonly used material is PDMS. Sun et al[23] replicated the surface morphology of a lotus leaf using PDMS. This negative template was then used to create a positive replica. This positive replicate matched the lotus leaf morphology to a very high degree. When observed under an SEM, this replica even contained the nano-textures along with the micro-textured that were seen on the lotus leaf. 
Another approach similar to templating is Nano-print lithography. In this technique a hard Master with the desired morphology is pressed onto a thermoplastic polymer material above the glass temperature of the polymer. A negative replica of the Master is obtained after cooling and removing the polymer layer gently.

\subsubsection{Plasma treatment of surfaces}

One of the Top Down approach is the plasma etching of surfaces. The ionized species in the plasma phase are accelerated towards the substrates creating deep grooves and steep walls. Few authors such as Fresnais et al[24] have shown the plasma etching could be used to induce roughness in low density Polyethylene. Plasma treatment with Oxygen and $-\mathrm{CF} 4$ yielded a surface with contact angle of 170degrees with low hysteresis. The RMS roughness induced by the plasma on such a surface was between 200 and $400 \mathrm{~nm}$.

One very interesting work to be looked into was the work done by Minko et al[25] They carried out plasma treatment on PTFE surfaces using Oxygen and obtained contact angles of $160^{\circ}$ with no hysteresis. One good thing that cannot be overlooked is that PTFE is a very low surface energy material and in combination with roughness produces very robust ultra-phobic surfaces.

One must remember that plasma treatment of surfaces for inducing roughness is a dry etching technique and rough surfaces can be easily obtained.

Bottom down approaches include all the techniques which involve the building of larger, more complex objects by the integration of smaller building blocks or components either through self- assembly or self-organization[22]. Some of the Bottom-up approaches 
include Chemical vapor deposition, Layer-by-Layer deposition by electrostatic assembly, colloidal assemblies, sol-gel techniques, and chemical synthesis.

2.1.4 Colloidal Assemblies: Another interesting technique in the development of superhydrophobic surfaces are Colloidal Assemblies. Roughness can be induced by the assembly of particles on underlying substrates. In one of the works, super-hydrophobic coatings were created by spin casting Mono-dispersed polystyrene beads[26] which assembly very closely once spinned. The roughness of these films was adjusted by using Oxygen plasma etching to control the size of these deposited particles. Gold deposition on these particles was carried out followed by octadecanethiol self assembled monolayers to render the entire surface hydrophobic.

Klein et al[27] have created super-hydrophobic surfaces by depositing Silica nanoparticles on alumina substrates. The substrates were annealed to partially sinter the nanoparticles to the substrates. The surfaces were later rendered hydrophobic by silanising them with a fluoroalkylsilane. Analysis of surface area fractions revealed that the contact angle increased with decreasing area fractions up to the point where the pressure of the water droplet causes the droplet to spontaneously wet the substrate.

2.1.5 Layer by Layer deposition(LBL): Another deposition technique in the Bottom up approaches is the Layer by Layer deposition(LBL)[22] which takes advantage of electrostatic charge interactions between different Poly-anion and Poly-cation layers. One advantage of this technique is that it allows precise control of the thickness. Roughness in these layers is induced by the incorporation of nanoparticles. In one of the works, Zhai et al[28] have prepared super-hydrophobic surfaces on silicon using Poly-allylamine 
hydrochloride(PAH)/poly(acrylic acid)(PAA) multi-layers via LBL deposition. The morphology of these surfaces consisted of pores in the order of 10um in a honeycomb like manner using appropriate acid treatments. Hydrophobicity was finally achieved by coating these films with a semi-fluorinated silane. The roughness hierarchy consisting of the micron sized pores due to the acidic treatments and the nano-sized roughness due to the incorporation of nano-particles rendered these surfaces super-hydrophobic.

Han et al[29] have created super-hydrophobic surfaces with PAA coated $\mathrm{ZrO} 2$ particles with PAH(Polyallylamine hydrochloride) consecutive layers on Silicon. The surface showed a water contact angle of 139 degrees and a hysteresis of 40degrees for a PAH exposed outermost layer after 20 depositions. The hysteresis was further decreased by the deposition of silica particle on the substrates and dipping the substrates in a fluorinated silanising agent. Amidation was used to render the films extra stability. The final films showed a water contact angle of 170 degrees and a hysteresis of 2 degrees.

As earlier stated, Layer by Layer deposition using Poly-electrolytes allows precise control of the thickness of the films[22]. A not so good thing about these polyelectrolytes is that poly-electrolytes are hydrophilic and additional steps are required to render them hydrophobic. Also for additional hydrophobicity often additional steps such as acid treatments, incorporation of nano-particles etc. are required[22] which makes the process not all that straight forward. 
2.1.6 Sol-gel methods too are some of the bottom-up approaches that are used for the creation of super-hydrophobic surfaces. The entire theory behind the sol-gel processes has been described in a later part of this thesis.

Shang et al[30] have used a sol-gel process in the development of a super-hydrophobic surface. Careful control over the hydrolysis and the condensation reactions was used to control the morphology and thus the surface roughness. The surface chemistry of these coatings were altered using monolayers that were introduced in the sol during the condensation reactions. Transparencies obtained by these coatings were higher than $90 \%$ with advancing and receding water contact angles of $165^{\circ}$ and $115^{\circ}$.

Hikita et al[31] have created super-hydrophobic surfaces using colloidal Silica particles and fluoroalkylsilanes. The silica nano-particles were used to control the surface roughness and surface energy. For one particular loading of silica nano-particles and for one particular Silica nanoparticle to fluoroalylsilane ratio, the films exhibited repellency to both water and oil. This method can be used for making very large surfaces superhydrophobic.

Y. Xiu et al[34] have created super-hydrophobic coatings using the sol-gel techniques. Their coatings were made porous with the help of a eutectic liquid as the templating agent. Contact angles as high as 161 degree with a hyteresis of 4.1 degrees were exhibited by these porous thin films.

$K$. Chang et al[35] created similar super-hydrophobic coatings using PEG(Polyethylene glycol) as the templating agent in creating porous silica coatings. Their contact angles were greater than 150degrees with transparencies above $90 \%$. 
E. Burkarter et al[32] have created super-hydrophobic surfaces by employing the electrospray technique using a commercial PTFE particle suspension in water. Water contact angles as high as $167^{\circ}$ were obtained on these surfaces. From the SEM images it is seen that such a surface had a very high roughness due to the agglomeration of particles forming a cauliflower like structure. Such a surface was thought to be replicable if the same morphology could be obtained with the aid of nano-particles.

L. Feng et al[33] have created surfaces exhibiting both super-hydrophobic as well as super-oleophilic characteristics using homogenous Teflon solutions. The morphology is seen to consist of ball like structures ranging from 2-5um as well as crater like structures in the range of $70 \mathrm{~nm}$. Hence roughness was seen to occur at two scales - the micro and nano scales. The crater like morphology was created with the help of surfactants while the ball like morphology was created by the addition of non-solvents causing the Teflon to precipitate forming the balls.

$H$ Erbil et al[36] have made polymer films such as polypropylene porous by first dissolving it in a good solvent such as o-xylene or decalin and then adding a non solvent to the solution. When such non-solvents are added, the polymers are seen to precipitate due to the increase in the polymer phase separation between the two phases of nonsolvent plus the good solvent and the polymer plus small amounts of the good solvent. Two macroscopic phases are seen at the completion of the process: one polymer rich and the other polymer poor[2]. It is seen that crystallization starts in the polymer rich phase by the formation of crystal nuclei with further development into spherulites, fibrilates and other crystal shapes. It is seen that the smaller spherulites resulted due to the increase in nucleation rate, due to the presence of non-solvents. Also since in most cases the non- 
solvents are more volatile than the solvents, faster evaporation leads to shorter times for crystal formation. Also at times it is seen that non-solvents containing oxygen increase the wettability of the polymer solution on the substrate due to the presence of $-\mathrm{OH}$ groups whenever glass substrates are used resulting in an initial homogenous layer over which the network grows forming a much more homogenous final coating. 


\section{CHAPTER III: SUPER-HYDROPHOBIC SURFACES}

\subsection{Introduction}

The wetting properties of a solid are determined by contact angle measurements of the liquid on a flat surface. The three parameters[37] that are required while investigating the wetting properties of a solid are i) the static contact ' $\theta$ ' - which is the contact angle of a droplet in equilibrium ii)the advancing contact angle $\theta_{\mathrm{a}}$, which is the increase in the contact angle as water is added to the droplet making sure that the total projected area occupied by the droplet does not change. iii) the receding contact angle $\theta_{\mathrm{r}}$ which is decrease in the contact angle from the equilibrium state by reducing the volume of the droplet making sure that the projected area of the droplet does not shrink as the water is sucked out.

If a surface on which the droplet is placed is tilted, the drop begins to move or slide as soon as the lower contact angle reaches the advancing angle[37] and the upper contact angle reaches the receding angle. The range of angle $\Delta \theta=\theta_{\mathrm{a}}-\theta_{\mathrm{r}}$ is refereed to the contact angle hyteresis. 


\subsubsection{Super-hydrophobic Surfaces}

Surfaces with water contact angles greater than $150^{\circ}$ are classified as super-hydrophobic or ultra-phobic surfaces. The contact angle that's observed is not the real contact angle and as such is termed as the apparent contact angle.

The fundamental principle for the creation of a super-hydrophobic surface[38] is the combination of hierarchical micro /nanostructures and low surface energy materials. The micro / nanostructures increase the surface area dramatically so that air can be trapped in the aperture of porous micro-spheres / nano-fibers used for creating the rough surface.

The contact angle of a drop on a solid is ideally(for a flat homogenous solid) given by the classical Young's equation[39]:

$$
\cos \theta=\frac{\gamma_{S V}-\gamma_{S L}}{\gamma}
$$

Equation 3-i

Where $\gamma_{S V}, \gamma_{S L}$ and $\gamma$ are the different surface tensions (solid/vapor, solid/liquid, and liquid/vapor) involved in the system.

A lot of studies have been done in modeling super-hydrophobic surfaces. Modeling of such surfaces can be used to predict the contact angle of a water droplet on such surfaces in most cases. The most widely used models are the models presented by Wenzel[40] and Cassie[41].

\subsubsection{Wenzel's Theory}

Wenzel proposed the first approach to characterize the influence of the surface roughness on the wettability on the solid.

$$
\cos \theta^{*}=r \cos \theta
$$

Equation 3-ii 
Where $\theta$ is the Young's angle and $r$ is the roughness factor which is given by the ratio of the actual area of solid in contact with the liquid to the projected area.

The Basic assumption in Wenzel's theory is that that liquid follows the roughness of the surface as shown in the diagram below. From the equation, there seems to be a linear relation between the apparent contact angle and the roughness factor. According to Wenzel's model, the roughness enhances both the hydrophobicity as well as the hydrophilicity depending on the nature of the flat surface. It should be noted that the Wenzel's law looses its integrity when the ratio ' $r$ ' becomes too large, as the maximum value of $\operatorname{Cos} \theta^{*}$ cannot exceed 1 .

The Wenzel equation defines the apparent contact angle as the angle that minimizes the surface energy of the drop during hypothetical displacement ' $d x$ ' of the contact line[39]. The surface energy change ' $d E$ ' associated with the contact line displacement ' $d x$ ' can be expressed by the equation

$$
d E=r\left(\gamma_{S L}-\gamma_{S V}\right) d x+\gamma_{L V} d x \operatorname{Cos} \theta_{W} \quad \text { Equation 3-iii }
$$

with respect to the contact line unit length. If $r=1$ (on a flat surface) the Young equation is obtained when in equilibrium and corresponding to a minimum of energy whereas the Wenzel law is obtained for a rough surface $(r>1)$. 


\subsubsection{Cassie-Baxter Theory}

Cassie and Baxter[43] developed the equation (a) to establish a relation between the Contact Angle(CA) of a water droplet on a flat $\operatorname{surface}(\theta)$ and the contact angle on a heterogeneous surface composed of a solid and air.

$$
\cos \theta_{C B}=\Phi_{1} \cos \theta-\Phi_{2} \quad \text { Equation 3-iv }
$$

Here ' $\Phi_{1}$ ' and ' $\Phi_{2}$ ' are the fractions of the solid surface and air in contact with liquid, respectively.

$$
\Phi_{1}+\Phi_{1}=1 \quad \text { Equation 3-v }
$$

In the Cassie-Baxter(CB) model, the apparent contact angle is a sole function of only the solid fraction for a given surface of $\mathrm{CA}$ ' $\theta$ '. Hence in designing a super-hydrophobic surface, the contribution of only a small faction of the solid is required to make this surface super-hydrophobic or a material with a very high contact angle needs to be used. In practice, the $\mathrm{CB}$ model cannot predict accurately the wetting behavior of a predesigned surface. It is used as a comparison with the measured result to show the presence of the droplet in the CB state.

In designing super-hydrophobic surfaces, only the hydrophobic domains of both the models are considered and plotted here. Since $r>1$, the apparent contact angle is $180^{\circ}$ when $\theta_{\mathrm{e}}=-1 / \mathrm{r}$. Hence the apparent contact angle turns out to be $180^{\circ}$ for $\theta_{\mathrm{e}}>\operatorname{Cos}^{-1}(-1 / \mathrm{r})$ according to Wenzel's theory. According to Cassie's theory, the apparent contact angle changes sharply at $\theta_{\mathrm{e}}=90^{\circ}$ and is $180^{\circ}$ only when $\theta_{\mathrm{e}}=180^{\circ}$. 


\subsection{Surface Energies}

The surface free energies and their components between two interacting surfaces[44] are extremely important since not only do they dictate the strength of interaction, but also control processes like the stability of aqueous colloidal suspensions, the dynamics of molecular self-assembly, wetting, spreading, deinking and adhesion. It is necessary to measure the contact angle $(\theta)$, if one wishes to characterize the surface of a solid in terms of its surface free energy components.

Surface energies were carried out with different probe liquids i.e. DI water, Diiodomethane, Formaamide. Four sets of data were recorded for each surface while carrying out the contact angle measurements. The data was plotted with the corresponding arrow bars for the different surfaces which have been displayed in the later chapters.

Surface free energies of the coatings were calculated based on the below equations which were obtained from[50]:-

$0.5 \gamma_{1}(1+\cos \theta)=\sqrt{\left(\gamma_{s}^{L W} \gamma_{1}^{L W}\right)}+\sqrt{\left(\gamma_{s}^{-} \gamma_{1}^{+}\right)}+\sqrt{\gamma_{s}^{+} \gamma_{1}^{-}}$

Equation 3-vi

The probe liquid diiodomethane(denoted as 1) is used to get the Lifshitz-van der Waals component of the surface free energy $\left(\gamma_{s}^{L W}\right)$

$\gamma_{s}^{L W}=0.25 \gamma_{1}^{L W}(1+\operatorname{Cos} \theta)^{2}$ Equation 3-vii

With the known $\gamma_{s}^{L W}$ value, the $\operatorname{acid}\left(\gamma_{s}^{+}\right)$and base $\left(\gamma_{s}^{-}\right)$parameters of the surface energy can be calculated from the contact angles of water(denoted as 2) and formamide(denoted as 3) 
$\sqrt{\gamma_{s}^{+}}=(\mathrm{AF}-\mathrm{BD}) /(\mathrm{CF}-\mathrm{CE})$ and $\sqrt{\gamma_{s}^{-}}=(\mathrm{BC}-\mathrm{AE}) /(\mathrm{CF}-\mathrm{CE})$

Equation 3-viii

Where $B=\gamma_{3}(1+\operatorname{Cos} \theta)-2\left(\gamma_{s}{ }^{L W} \gamma_{3}{ }^{L W}\right)$

$A=\gamma_{2}(1+\operatorname{Cos} \theta)-2\left(\gamma_{s}{ }^{L W} \gamma_{2}{ }^{L W}\right)$

$C=2 \sqrt{\gamma_{2}^{-}} \quad D=2 \sqrt{\gamma_{2}^{+}}$

$E=2 \sqrt{\gamma_{3}^{-}} \quad F=2 \sqrt{\gamma_{3}^{+}}$

The total acid base components of the surface free energy are given by $\gamma_{s}^{A B}=2 \sqrt{\gamma_{S}^{-}} \sqrt{\gamma_{S}^{+}}$

Equation 3-ix

And the total surface free energy of the film coating sample is given by

$$
\gamma_{s}=\gamma_{S}{ }^{L W}+\gamma_{S}{ }^{A B}
$$

Equation 3-x 


\subsection{The Sol-gel Chemistry}

\subsubsection{The Theory}

The sol-gel process uses high purity monomers [45] for low-temperature production of fibers, monoliths, coatings and powders. A number of variables influence the structural evolution of silicate polymers: $\mathrm{pH}$, solvent, Water- to-Silicon ratio(W) and monomer.

The two fundamental chemical reactions of the sol-gel process are (1) hydrolysis

$$
\equiv S i-O R+\mathrm{H}_{2} \mathrm{O} \underset{K_{E}}{\stackrel{K_{H}}{\rightleftharpoons}} \mathrm{Si}(\mathrm{OH})+\mathrm{ROH} \quad \text { Equation 3-xi }
$$

and (2) Condensation

$\equiv S i-O R+H O-S i \equiv \stackrel{K_{A}}{\longleftarrow} \equiv S i-O-S i \equiv+$ ROH(alcohol producing)

Equation 3-xii

$\equiv \mathrm{Si}-\mathrm{OH}+\mathrm{HO}-\mathrm{Si} \equiv \stackrel{K_{W}}{\rightleftharpoons} \equiv \mathrm{Si}-\mathrm{O}-\mathrm{Si} \equiv+\mathrm{H}_{2} \mathrm{O}$ (water producing)

\section{Equation 3-xiii}

Where $\mathrm{K}_{\mathrm{H}}, \mathrm{K}_{\mathrm{E}}, \mathrm{K}_{\mathrm{A}}$, and $\mathrm{K}_{\mathrm{W}}$ are rate constants for hydrolysis, esterification, alcohol producing condensation, and water producing condensation respectively. Usually hydrolysis and condensation reactions are concurrent.

Starting generally with alcoholic solutions of monomeric metal alkoxide precursors[45] $\mathrm{M}(\mathrm{OR})_{\mathrm{n}}$ where $\mathrm{M}$ represents a network forming element such as $\mathrm{Si}, \mathrm{B}$, Ti, etc., and $\mathrm{R}$ is normally an alkyl group. $\left(\mathrm{C}_{\mathrm{x}} \mathrm{H}_{2 \mathrm{x}+1}\right)$, water plus acid or base catalyst are added in sufficient quantities that the $\mathrm{H}_{2} \mathrm{O} / \mathrm{M}$ molar ratio, $\mathrm{r}$, varies from 1 to over 50. The net hydrolysis reaction replaces alkoxide groups with hydroxyl groups and generates the byproduct alcohol. Hydrolysis species undergo one of two condensation reactions to produce Si-O-Si bonds plus alcohol or water as by-products. 
The evolution of the Si-O-Si network can proceed along many different pathways[46] depending on processing parameters such as $\mathrm{pH}$, $\mathrm{r}$, solvent, and nature of the catalyst. Gelation occurs when the network that forms spans the entire solution volume, regardless of the specific structure of the solution species. At the gel point, the sudden increase in solution viscosity in effect freezes in a structure characteristic of the solution conditions.

Prior to gelation[45], the solution species may be deposited on a variety of substrates by dipping, spinning or spraying to form thin films. Structural evolution of films occurs on a much different time scale than in bulk gels, because gelation and drying take place in seconds rather than days or months. In general the structures of films depend on the extent of branching or aggregation of the solution precursors and the relative rates of condensation and evaporation during film formation. Because the films are thin and rigidly adhere to the substrate, their consolidation occurs one-dimensionally.

\subsubsection{Templating using Organic Materials}

The development of mesoporous materials has long been studied since the discovery of M41S family of mesoporous molecular sieves[47]. The mesoporous materials of the classic M14S family are generally obtained using surfactants such as primary amines and quaternary ammonium ions. These products are formed by the electrostatic attraction between the ceramic precursor and the surfactant upon which the surfactant rich gel phase precipitates from the heterogeneous reaction mixture. Templating materials such as micro-emulsions, block co-polymers, latex particles, and bacterial superstructures were employed for pore formation and control. 
Various surfactants such as $\mathrm{CTAB}$ (Cetyltrimethlyammonium bromide), poly(sodium 4styrene sulphonate)/PSS, Pluronics P123, F127 are among the few which have been used by different researchers in the templating of silica based sol-gel films.

Usually after the sol-gel films have been completely cured, a final step of silanising them with a low surface energy silane is followed to render them hydrophobic.

A brief description is given below on the theory behind Silane coupling agents and their mechanism to substrate bonding.

\subsection{Silane Coupling Agents}

Silane coupling agents are silicon-based chemicals that contain two types of reactivity[48] - inorganic and organic - in the same molecule. A typical general structure is $(\mathrm{RO})_{3} \mathrm{SiCH}_{2} \mathrm{CH}_{2} \mathrm{CH}_{2}-\mathrm{X}$, where $\mathrm{RO}$ is a hydrolyzable group, such as methoxy, ethoxy, or acetoxy, and $\mathrm{X}$ is an organo-functional group, such as amino, methacryloxy, epoxy, etc.

A silane coupling agent will act at an interface between an inorganic substrate (such as glass, metal or mineral) and an organic material[48] (such as an organic polymer, coating or adhesive) to bond, or couple, the two dissimilar materials.

\subsubsection{The Silane Bond to the Inorganic Substrate}

Silane coupling agents that contain three inorganic reactive groups on silicon[48] (usually methoxy, ethoxy or acetoxy) will bond well to the metal hydroxyl groups on most inorganic substrates, especially if the substrate contains silicon, aluminum or a heavy metal in its structure. The alkoxy groups on silicon hydrolyze to silanols, either through the addition of water or from residual water on the inorganic surface. Then the silanols 
coordinate with metal hydroxyl groups on the inorganic surface to form an oxane bond and eliminate water.

Silane molecules also react with each other to give a multi-molecular structure[48] of bound silane coupling agents on the surface. More than one layer, or monolayer equivalents, of silane is usually applied to the surface. This results in a tight siloxane network close to the inorganic surface that becomes more diffuse, away from the surface.

HMDS(1,1,1,3,3,3-hexamethyldisilazane) is one of the hydrophobic silanes that was used to silanise the surface of our sol-gel based films so that these films could become hydrophobic. 


\subsection{Surface Characterizations}

The surfaces were characterized using contact angle measurements with water. SEM(Sanning Electron microscopy) and AFM(Atomic Force microscopy were used to observe surface morphology. UV-VIS spectroscopy was used to measure film transparency. Fourier transform infrared spectroscopy(FTIR) was used to analyze the presence of chemical bonds/species within the films.

\subsubsection{Contact Angle measurement system}

For our contact angle measurement system, a high speed Fire-Wire Digital Video camera 'Sony DFW SX-900' was used to capture the drop images on the substrates under test. An X-Y stage was used on which the substrates were mounted. The backside of the stage was illuminated with the help of a lamp. De-ionized water was used in all cases for the water contact angle measurements. The sessile drop method was used to measure the contact angle each time. The water was manually dropped with the help of a pipette. Each time a drop volume of $5 \mathrm{ul}$ was used. Once the images were captured on the computer using the 'National Instruments Acquisition Software', the images were processed for contact angle measurements using the 'ImageJ' software.

The 'ImageJ' software requires the user to demarcate the extremities of droplet on the image window along with some intermediate point along the liquid air droplet boundary. It then joins all the points together and draws the tangent to the surface of the droplet. It then calculates the angle of the tangent which becomes the contact angles of the liquid. 


\subsubsection{Scanning Electron Microscope}

The surface morphology was observed using either a Hitachi S4700 FESEM or a Quanta FEG 600 ESEM. A very thin carbon film of the order of 2-5nm was evaporated on the samples when the Hitachi S4700 FESEM was used. This was done to prevent charge buildup during the scanning. In most cases a $5 \mathrm{kV}$ or a $10 \mathrm{kV}$ beam was used during the scanning.

\subsubsection{Atomic Force Microscopy}

Atomic Force microscopy was performed using the Agilent 5100 AFM system. The sample was imaged in the non-contact mode/“tapping" mode using Silicon tips purchased from NANOSENSORS. Super Sharp Silicon tips, model \# SSS-NCHR having a sprint constant of $42 \mathrm{~N} / \mathrm{m}$ and resonant frequency of $330 \mathrm{Khz}$ were used for the imaging. The obtained SEM images were analysed with Gwyddion which is a modular program for SPM (scanning probe microscopy) data visualization and analysis. Primarily it is intended for analysis of height fields obtained by means of scanning probe microscopy techniques (AFM, MFM, STM, SNOM/NSOM), however it can be generally used for any other height field and image analysis. 


\section{CHAPTER IV: SUPER-HYDROPHOBIC SURFACES- FABRICATION}

The Sol-gel approach was the first approach that was used in the development of superhydrophobic coatings. Roughness in such coatings could be induced either by the addition of nano-particles or simply by making the coatings porous with the help of templating agents. In our approach which was similar to the approaches as followed by $K$. Chang* and Y. Xiul*, templating was chosen to create such porous structures. Organic ${ }^{*}$ surfactants such as $\mathrm{CTAB}($ Cetyltrimethlyammonium bromide) were chosen as the templating agents.

A brief description of the science behind the Sol-gel process has been described in Chapter 3.

\subsection{Porous Silica Sol-gel Experimental Procedure}

The experimental procedure was the same as followed by $Y$. Lu et al[51], the details of which have been described below.

Materials: TEOS(Tetraethyl OrthoSilicate) of purity $>99 \%$, Ethanol of purity $>98 \%$ and $\mathrm{HCl} 36 \%$ were purchased from Sigma-Aldrich. The materials were used as received. No further purification of the materials were carried out on them.

\footnotetext{
* Described in the literature review section in chapter 2
} 


\subsubsection{Preparation of Sol-gel Silica Films}

For the pre-hydrolysis step, TEOS $\left(\mathrm{C}_{8} \mathrm{H}_{20} \mathrm{O}_{4} \mathrm{Si}\right)$, Ethanol $\left(\mathrm{C}_{2} \mathrm{H}_{5} \mathrm{OH}\right), \mathrm{HCL}$ and DI water are taken in the $1: 3: 5 \times 10^{-5}: 1$ molar ratio which translates to $55.82: 46: 1 \times 1^{-3}: 4.5$ milliliters and heated under reflux at $60 \mathrm{C}$ for $1 \mathrm{hr} 15 \mathrm{~min}$. The pre-hydrolyzed solution is then taken and $\mathrm{CTAB}\left(\mathrm{C}_{19} \mathrm{H}_{42} \mathrm{BrN}\right), \mathrm{EtOH}$ and DI water are progressively added to get to the final molar ratio of TEOS : EtOH : HCL : DI water : CTAB to $1 \mathbf{: ~ 1 0 - 4 0 : 0 . 1 5 - 0 . 0 0 4}$ : 5 : 0.8-0.35 which translates to 203-1089ml : 6.15-0.162ml : 36ml : 14-63gm respectively.

The range for the HCL volumes determines how long we have to age the samples. 2D Hexagonal closed pack structures are favored when the CTAB/Si ratio is kept at 0.14 . After the pre-hydrolysis step, the mixture is stirred at $25 \mathrm{C}$ for $15 \mathrm{~min}$. The solution is then aged for $15 \mathrm{~min}$ on a hot bath.

This solution is then used to dip coat either Silicon or Glass substrates using a Dip-Coater at different dip coating speeds. Different dip coating speeds were used to control the thickness of the films. The dip-coated films were placed on a hot plat at $130 \mathrm{C}$ for $2 \mathrm{~min}$ to eliminate most of the solvent present and then placed in an oven at $430 \mathrm{C}$ for $1 \mathrm{hrs}$. The temperature of the oven was raised at a ramp rate of 1 degree $\mathrm{C} / \mathrm{min}$.

After having cooled down the films were characterized using UV-VIS Ellipsometry spectroscopy, FTIR(Fourier transform infrared spectroscopy) and contact angle measurements with water.

Simultaneously a process was being developed for the low temperature processing of these sol-gel films. This was done so that the hydrophilic coatings developed could be applied onto plastic substrates such as Polymethyl methacrylate(PMMA) or Polyethylene 
terephthalate(PET) where by the maximum temperature at which the process could be carried out at was limited to $150 \mathrm{C}$.

\subsubsection{Low Temperature processing of films}

A process for curing our sol-gel based porous silica films at low temperatures was being developed in our lab. For this we had to make sure that our process could effectively get rid of the surfactants viz. $\mathrm{CTAB}$ (Cetyl trimethylammonium bromide) used for the templating, from the films. We also had to make sure that the silica precursor TEOS(tetra-ethyl-ortho-silicate) could cross-link well enough that would result in a hardy film. For effective cross-linking to take place[56], the hydrolyzed species would have to condense resulting in the Si-O-Si Siloxane bonds. The same procedure was followed as in the case of the high temperature processed films except that the processing temperature was limited to $150 \mathrm{C}$ in an oven for either $0 \mathrm{hrs}, 6 \mathrm{hrs}$ and $12 \mathrm{hrs}$ after the evaporation of organic solvents on a hot-plate at $100 \mathrm{C}$ for 5 minutes.

The As-prepared low temperature sol-gel thin films were treated with $\mathrm{O}_{2}$ plasma as before for various exposure times; the conditions of which have been specified below. The plasma treatment was performed in M/s Harrick Plasma (Model PDC-32G). Low RF power of $12 \mathrm{~W}$ was used for $\mathrm{O}_{2}$ plasma treatment with the following parameters: Oxygen flow was maintained at $180 \mathrm{SCCM}$ and a base pressure of $900 \mathrm{mTorr}$ for a specified amount of time. Thin films with different exposure times were evaluated for their hydrophilic behavior by carrying out contact angle measurements with water. The results have been summarized in the next chapter. 


\subsection{Nano-materials synthesized}

One way of inducing roughness in thin films is by the incorporation of nano-materials or nano-fillers in the matrix materials. In our study, only silica sol-gels and polymers such as Teflon and THV(a polymer of tetrafluoroethylene, hexafluoropropylene and vinylidene fluoride) were used as the binders into which these nano-materials were embedded. Various Nano-tubes, nano-rods and nano-particles are some of the different materials that are used as roughness inducing agents.

Some of the nano-materials that were created in our labs which were used as roughness inducing agents for our coatings have been cited below.

\subsubsection{Preparation of Organo-silicate(PMSSQ) Nano-rods}

Materials: PPG(Poly-Propylene-Glycol) with Molecular weight 425, PGMEA(Propylene-Glycol-Methyl-ether-acetate), MTMS - methyl tri-methoxy silane were purchased from Sigma Aldrich. PMSSQ(poly(methylsilsesquioxane)) was purchased from Techneglas. All chemical were used as received without any purification. Experimental Procedure: In this experiment, a 10 day old PPG(PolyPropyleneGlycol) / PMSSQ (poly(methylsilsesquioxane)) gel was used for the synthesis of the nano-rods, the details of the gel synthesis have been described elsewhere[52]. The gel was scooped from a vial(approx 3gm) and washed continuously with ethanol with the help of a filter paper(Fischer brand) and a beaker. The scooped gel was first pulverized manually in a beaker with a spoon to break down the matrix into smaller fragments. Ethanol was then added to the beaker and the solution was stirred manually for few minutes before filtering the solution containing the gel and decanting the excess ethanol. This procedure was 
repeated 4-5 times before collecting the final product from the filter paper. This washing is done to get rid of all the PPG(PolyPropylene Gylcol) in the matrix.

The ethanol-washed product was then taken in a ceramic/porcelain crucible and heated in a furnace at $450 \mathrm{C}$ for $3 \mathrm{hrs}$. The temperature was ramped at a rate of $10 \mathrm{C} / \mathrm{min}$ before allowing it to dwell at $450 \mathrm{C}$ for $3 \mathrm{hrs}$. The as-annealed product was characterized using TEM analysis.

\subsubsection{Preparation of PMSSQ Nano-particles}

Experimental Procedure: For the preparation of PMSSQ nanoparticles, 5gm PMSSQ is taken and added to $5 \mathrm{gm} \mathrm{EtOH}$ in one vial. The solution is then sonicated for about $5 \mathrm{~min}$ till the PMSSQ completely dissolves in EtOH. In another vial, 5gm PPG(Polypropylene glycol) is taken and added $5 \mathrm{gm}$ EtOH. The solution is sonicated for about $2 \mathrm{~min}$. The solutions from both these vials is then taken and added in a third vial. The mixture is sonicated for a minute or two. This solution is then put in a beaker on a hot plate at 72 degrees and evaporated continuously till about $2 \mathrm{gm}$ is evaporated. The solution is then cooled to about room temperature. A small part of this solution(approx 1gm) is taken and added drop-wise to DI water. This solution forms a precipitate on the walls of the vial. The water is drained off and the precipitate $(90 \mathrm{mg})$ from the walls of the vial is collected and re-dissolved in 6gm of EtOH. $1 \mathrm{gm}$ of this Ethanol solution is taken and added to $5 \mathrm{gm}$ of DI water to obtain a clear transparent solution. The particles nucleate and grow over time resulting in the spherical particles that were observed in the range $50-200 \mathrm{~nm}$. 


\subsection{Loading sol-gel films with organo-silicate nano-rods}

\subsubsection{Experimental procedure}

In another experiment, the PMSSQ nano-rods that were obtained by the washing and the high temperature treatment were embedded in sol-gel based films(the procedure of which has been cited below) to see if the desired roughness could be induced because of these nano-rods. The obtained films were transparent but the contact angles obtained with water were no better than the control samples(the sol-gel films without the nano-rods).

As earlier mentioned, the sol-gel solutions were loaded at $0.5 \mathrm{wt} \%, 1 \mathrm{wt} \%, 2.5 \mathrm{wt} \%$, and $5 \mathrm{wt} \%$ and the corresponding films were characterized by contact angle measurements. A different procedure was followed for the sol-gel synthesis[53] which has been described below.

Materials: The same materials were used as described in the earlier section for the solgel synthesis.

\subsubsection{Experimental procedure for Sol-gel synthesis}

The sol-gel precursor preparation and hydrolysis were performed sequentially in a controlled-temperature environment. For the sol-gel process, precursor solutions were prepared under acidic conditions in two steps. The precursor solution was diluted with ethanol to prolong the gelling time so that a thin film of this solution could be spun on a substrate. In step, one a typical starting mixture containing TEOS (tetraethoxysilane, $\left.\mathrm{Si}(\mathrm{OEt})_{4}\right)$, MTMS (methyltrimethoxysilane, $\left.\mathrm{Si}(\mathrm{OMe})_{3} \mathrm{CH}_{3}\right)$, water, $\mathrm{HCl}$, and anhydrous ethanol $(99 \%)$ was heated at $60^{\circ} \mathrm{C}$ for $90 \mathrm{~min}$. The amount of MTMS and TEOS were mixed in a $50 \mathrm{~mol} \%$ basis. A total of $0.25 \mathrm{~mol}$ of water were added per alkoxy group, 
and ethanol and $\mathrm{HCl}$ were added in molar ratios of 3 and $5 \times 10^{-5}$ relative to the silicate precursors thus hydrolyzing the silicates partially, while preventing significant condensation. This mixture was then diluted with ethanol, water and $\mathrm{HCl}$. The final molar ratios were typically: silicate(MTMS and TEOS):ethanol: $\mathrm{H}_{2} \mathrm{O}: \mathrm{HCl}=1: 20: 5: 3$ $\mathbf{x} 10^{-3}$. The prepared precursor solution was allowed to age for about three days for further poly-condensation. Several pre-cleaned glass substrates were drop casted with the different sol-gel organo-silicate mixture in different loading percentages and the films were heated to $60^{\circ} \mathrm{C}$ for 15 minutes for solvent removal and hardening. Contact angles were measured after annealing the samples at 400C.The same films were $\operatorname{HMDS}(1,1,1,3,3,3$-hexamethyldisilazane) treated in a pressurized chamber for 30 minutes at $110 \mathrm{C}$. The contact angles were measured again. 


\subsection{Teflon Thin Films}

4.4.1 PTFE(Poly Tetra Fluoro Ethylene) is a polymer that has a very low surface energy. This makes it hydrophobic giving it a water contact angle of 116-120 degrees. By inducing the roughness at the micro and nano scales, the water contact angle was shown to increase substantially making it super-hydrophobic. Roughness could be induced by the incorporation of micro and nano sized particles in the Teflon matrix(which will be described in detail in the later chapters) or by the creation of porosity in these Teflon films using micro-emulsions. Again since the roughness required, needed to be at two scales, nano-particles were introduced in the Teflon matrix creating the nano roughness.

Different approaches which were tried have been summarized below. Obtaining the right parameters such as the Teflon solution percentage, the amount of loading of particles required and the permissible thickness of the films was a major challenge in the development of these super-hydrophobic films. 


\subsubsection{Porous polymer films using micro-emulsions}

$H$ Erbil et $a l^{*}$ have made polymer films such as polypropylene porous by first dissolving it in a good solvent such as o-xylene or decalin and then adding a non solvent to the solution.

Since alcohols are immiscible in Teflon solvents such as FC-770 which is used to dissolve the amorphous Teflon pellets, they were the first choice of non-solvents in the micro-emulsion process. Water too was chosen to see its effect as a non-solvent in the micro-emulsion process in a Teflon solution.

\subsubsection{The Micro-emulsion Process:}

\section{Experimental procedure}

Using the micro-emulsion process, porosity was created in Teflon films. Different immiscible solvents were initially used. Ethanol was later chosen and different weight percentages were used to obtain the pores. The results have been summarized in the Results section of this thesis. The films were characterized using Optical Microscopy. UV-VIS spectroscopy was also used to measure transmittance characteristics of these films.

\subsection{Teflon AF 1600 films embedded with SiC Nano-particles}

P. Manoudis et al[55] have prepared super-hydrophobic surfaces with the aid of Silica nano-particles. They add 7 and 14nm Silica nano-particles to a PMMA (Poly(methyl methacrylate) solution and use this solution to coat marble specimens whose surfaces

\footnotetext{
* Described in the chapter 2
} 
they were trying to render super-hydrophobic. They achieved water contact angles as high as 157 degrees. The polymers act as binding media[55] between the nano-particles and the substrate.

A similar approach was used to make such coatings using Silicon Carbide nano-particles. The polymer we chose was PTFE or amorphous Teflon 1600 for reasons cited above. The experimental details have been cited below.

Materials: Amorphous Teflon AF 1600(pellets) was purchased from DuPont. Fluorinert FC-75, (also known as FC-770) which is used as the solvent was purchased from 3M. FluoroSyl FSM 660 which is a perfluorooctal mono-functional trimethoxy silane was purchased from Cytonix corporation, Beltsville, MD. Silicon Carbide nano-particles were purchased from 'Nanostructured and Amorphous Materials, Inc'(www.nanoamor.com).

\subsubsection{Experimental procedure}

Amorphous Teflon AF 1600 (pellets) is taken and a $2 \%(\mathrm{wt} \%)$ solution is made using FC75 as the solvent. The solution is processed by sonicating the mixture of the teflon pellets and the solvent under the application of heat at $60 \mathrm{C}$ for $6 \mathrm{hrs}$ till a clear, transparent solution is obtained. Before the spin-coating of this solution on a wafer, the wafer needed to be treated with an adhesion promoter so that the teflon could spread well on the surface and have good adhesion. A 5\%(wt\%) solution of FSM-660 is made with ethanol. This solution is spin coated on the wafer(either a glass or Silicon wafer) after a thorough cleaning of the wafer using a Piranha cleanse. The glass substrates that were used in this experiment were first Piranha cleaned to increase the $-\mathrm{OH}$ content on the glass surface. The wafer is then rinsed in DI water after the Piranha cleanse and then dried in an oven at 
75C.The FSM solution is spun at 2000RPM on the glass substrates. After a 5\% FSM solution is spin coated on the glass substrates, the substrates are heated at $110 \mathrm{C}$ for 10 minutes so that these FSM molecules could bond with the glass surface via $-\mathrm{OH}$ condensation reactions thus forming monolayers of FSM on the glass surfaces.

Before the addition of SiC NP's to the Teflon solution, the SiC NP's are FSM-silanised by adding them to a 5\%(wt\%) FSM-660/ethanol solution and then sonicating them for an hour before heating the dispersion to $110 \mathrm{C}$ till all the solvent evaporates leaving behind the FSM-silanised SiC NP's. FTIR analysis revealed that these NP's have a lot of $-\mathrm{OH}$ states on the surface as the spectrum consisted of a peak around $3500 \mathrm{~cm}^{-1}$ and hence FSM-silanising them proved to be a good idea which helped in obtaining better dispersions. A $0.1 \%(\mathrm{wt} \%)$ of these $130 \mathrm{~nm}$ FSM-silanised $\mathrm{SiC}$ particles are then added to this $2 \%(w t \%)$ Teflon solution and sonicated for 3 hrs till a homogenous dispersion is obtained. This SiC-dispersed Teflon solution is then spin-coated on the FSM coated glass substrates. The substrates are then heated successively on a hotplate at $125 \mathrm{C}$ for 15 minutes, $225 \mathrm{C}$ for 15 minutes followed by annealing at $300 \mathrm{C}$ for $2 \mathrm{hrs}$. The substrates are allowed to cool on the hotplate before any further tests are carried out on them.

In one of the experiments, a $0.5 \%(\mathrm{wt} \%)$ Teflon solution was prepared using the same procedure as mentioned earlier using $\mathrm{FC} \mathrm{-} 75$ or $\mathrm{FC}-770$ as the solvent. These solutions were loaded with different amounts of SiC Nano- particles to see the optimum loading conditions which create surface roughness while maintaining the transmittance. 


\subsubsection{Experiment with the Silicon-Oxy-Carbide Intermediate Layer}

In another experiment, an underlying layer of Silicon-Oxy-Carbide was deposited on a freshly Piranha cleaned glass substrate. Fluorine was incorporated into this layer in a gradient like manner from the bottom to top. The incorporation of fluorine helps in promoting better adhesion of Teflon to the substrate. It plays the same role as FSM by reducing the surface energy and thus helping in better spreading and wettability of the Teflon solution on the substrate. This thickness of this Silicon-Oxy-Carbide layer was about $650-1000 \mathrm{~nm}$. The surface energy of this coating was measured using the procedure described in chapter 3 .

\subsubsection{Experiment with the $0.125 \%(w t \%)$ Teflon configuration}

In another experiment a $0.125 \%(w t \%)$ Teflon Solution was prepared using the same procedure as above. These solutions were loaded with different $\mathrm{SiC}$ nano-particle loadings as before. This approach was carried out to see if the surface roughness could be enhanced with thinner coatings for the same $\mathrm{SiC}$ nanoparticles content. Very interesting results were obtained.

In another part of this experiment, samples were prepared wherein 3 layers were spinned consecutively on the same substrate. This was done to see if the roughness due to the $\mathrm{SiC}$ nanoparticles could be enhanced without causing too much change in the thickness of the film as compared to films produced by just a single coat. The Teflon solution percentage was kept the same. 


\subsection{Hardness Measurements}

Hardness of the films was measured by performing nano-indentation in an Agilent SPM model 5500. A diamond coated tip was used for creating dents on the sample applying a certain force. After the dent was created, the surface was imaged using the same tip in contact mode. The diameter of the dent is measured using the 'picoview' software. From the diameter and the force, hardness of the sample was measured using the equation, $H=P_{\max } / S$

\section{Equation 4-xiv}

Where $\mathrm{H}$ is hardness, $\mathrm{P}_{\max }$ is maximum force and $\mathrm{S}$ is area of the dent.

All the films were characterized using water contact angles, AFM and SEM. The results have been presented and discussed in the 'Results section' in chapter 5 of this thesis. 


\section{CHAPTER V: RESULTS AND DISCUSSION}

\subsection{Porous Silica Sol-gel Films}

The porous silica Sol-gel films were prepared as per the procedure as mentioned in Chapter 4. The high-temperature processed films were characterized using contact angle measurements and Spectroscopic Ellipsometry- the results of which have been summarized in table 5-1.

\subsubsection{The effect of Oxygen Plasma}

Before developing a low temperature process at which the films could be cured, a study on the effect of the Oxygen plasma* on the films was carried out to see if the hydrophilicity of the films could be further enhanced. It was seen that the OxygenPlasma ${ }^{*}$ treatment of high temperature processed films increased the hydrophilicity of the films when carried out for a certain duration of time as compared to films in the absence of such a treatment. This was confirmed by contact angle measurements before and after the application of the Oxygen Plasma*.

\footnotetext{
${ }^{*}$ Conditions of Oxygen Plasma cited in experimental section in chapter 4
} 
High Temp. processed
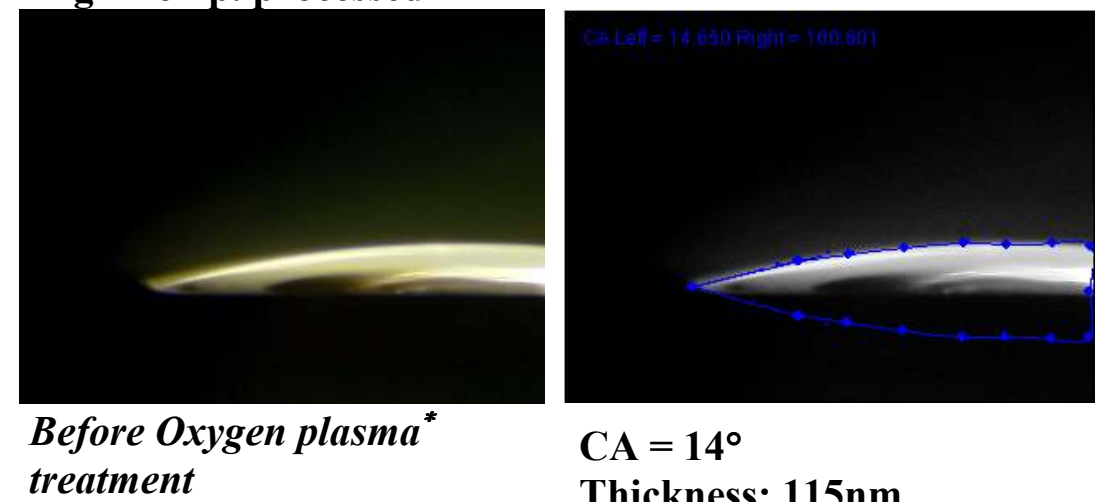

$\mathrm{CA}=14^{\circ}$

Thickness: $115 \mathrm{~nm}$

$n=1.24 @ 630 \mathrm{~nm}$ MSE 3.14

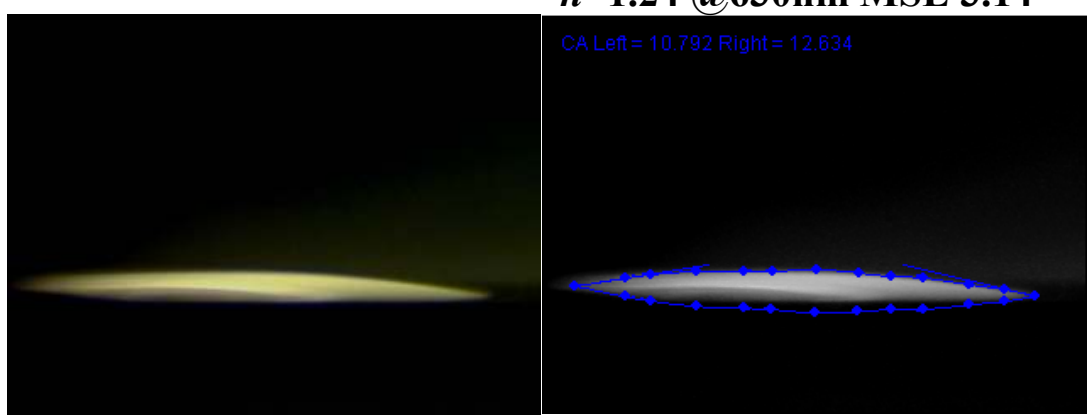

After Oxygen Plasma ${ }^{*} \quad \mathrm{CA}=10^{\circ}$

treatment

Table 5-1 Before and after Oxygen Plasma treatment(1min) and water immersion for 10min

As can be seen, the contact angles with water were seen to decrease from $14^{\circ}$ to $10^{\circ}$ indicating that the surface has become more super-hydrophilic. It is speculated that an oxygen plasma* results in the surface achieving more $-\mathrm{OH}$ groups thus increasing the surface energy of the film surface. It is also speculated that the surface silanol groups get enhanced at the cost of the Siloxane Si-O-Si bonds breaking up. The surface is rendered reactive due to the presence of dangling bonds immediately after the Oxygen plasma* When immersed in water, it is believed that the $-\mathrm{OH}$ groups in the liquid react with these dangling bonds creating the Silanol groups on the surface.

\footnotetext{
${ }^{*}$ Conditions of Oxygen Plasma cited in experimental section in chapter 4
} 
The effect of Oxygen plasma* on the hydrophilicity of the films with different exposure times was studied.

\section{Table depicting the variation in contact angle with Exposure time}

\begin{tabular}{|c|c|}
\hline Exposure Time(seconds) & Contact Angle(degrees) \\
\hline $60 \mathrm{sec}$ & 15.13 \\
\hline $50 \mathrm{sec}$ & 12.8 \\
\hline $40 \mathrm{sec}$ & 13.3 \\
\hline $30 \mathrm{sec}$ & 13.6 \\
\hline $20 \mathrm{sec}$ & 21.4 \\
\hline $10 \mathrm{sec}$ & 16.8 \\
\hline
\end{tabular}

Table 5-2

The above contact angles could further be decreased by silanisation with appropriate hydrophilic silanising agents. Before the silanisation, it was necessary to see if these results could be reproducible via a low temperature route. The next section talks about the characterization of the low temperature processed films. 


\subsection{Characterization of Low temperature processed films}

For the low temperature processing of the films, we had to make sure that our process could effectively get rid of the organic templates such as the surfactants viz. CTAB(Cetyl trimethylammonium bromide) used for the templating, from the films. We also had to make sure that the silica precursor TEOS(tetra-ethyl-ortho-silicate) could cross-link well enough that would result in a hardy film. For effective cross-linking to take place, the hydrolyzed species would have to condense completely resulting in the Si-O-Si Siloxane bonds.

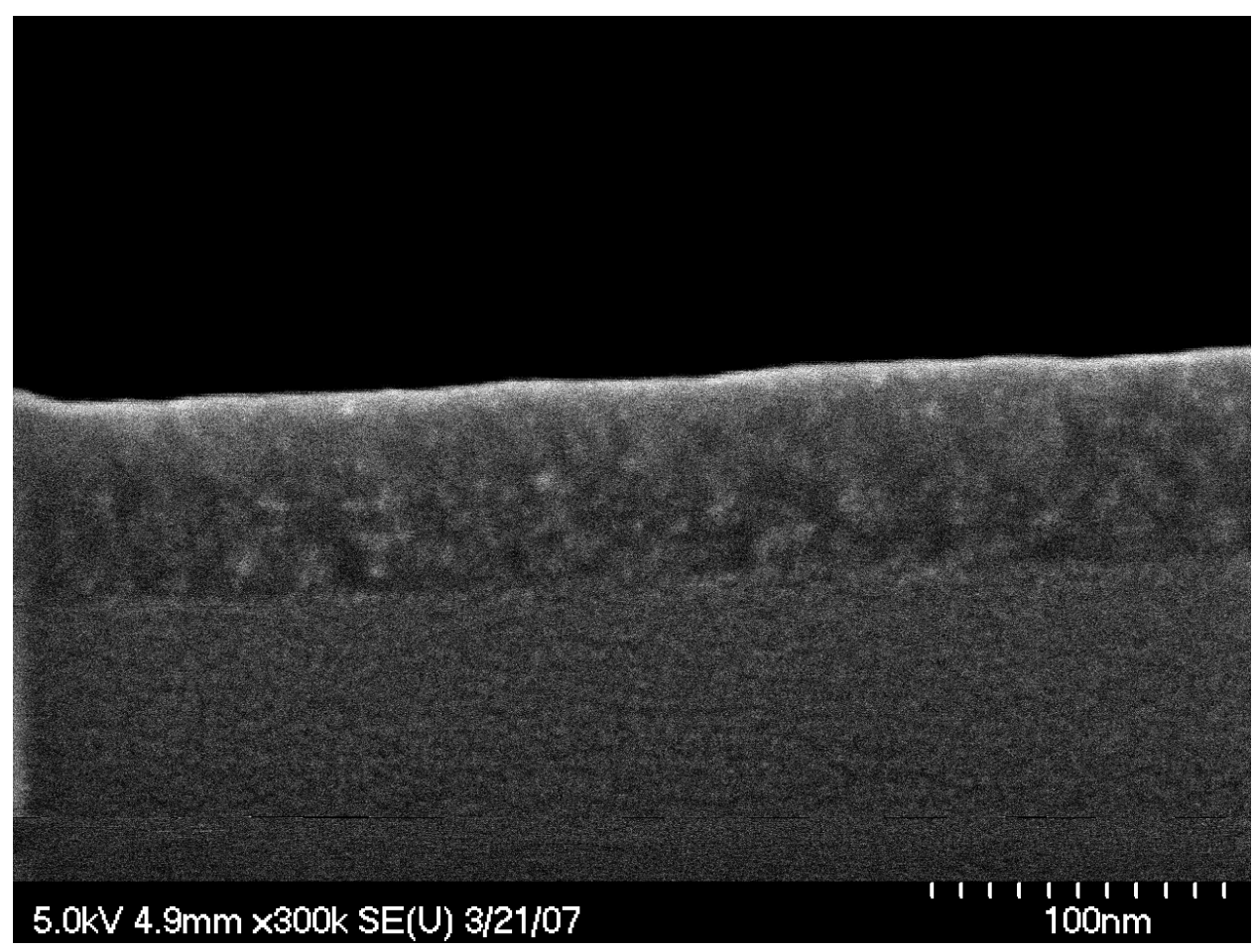

Figure 5-1 SEM depicting the structural changes that are brought about in low-temperature processed sol-gel films when exposed to Oxygen Plasma.

As can be seen in the above image, the first few $50 \sim 70 \mathrm{~nm}$ appear to have a slightly different texture as compared to the rest of the film. This is due to the damage induced as a result of the Oxygen plasma. Since the Si-O-Si network has not yet entirely cross- 
linked, the [57]damage is induced as a result of the Oxygen plasma. From the SEM image, the upper layer appears to be more denser than the bottom layer above the substrate.

The low temperature processed films were baked at $130 \mathrm{C}$ for $0 \mathrm{hr}, 12 \mathrm{hr}$ and $24 \mathrm{hrs}$ respectively. Below are the contact angles of the low temperatures processed films before and after the application of Oxygen plasma without any post processing.

\subsubsection{NO Annealing*(0Hr Annealing)}

\section{Thickness: 160nm MSE: 14.4}

\section{$\mathrm{RI}=1.49 @ 630 \mathrm{~nm}$}
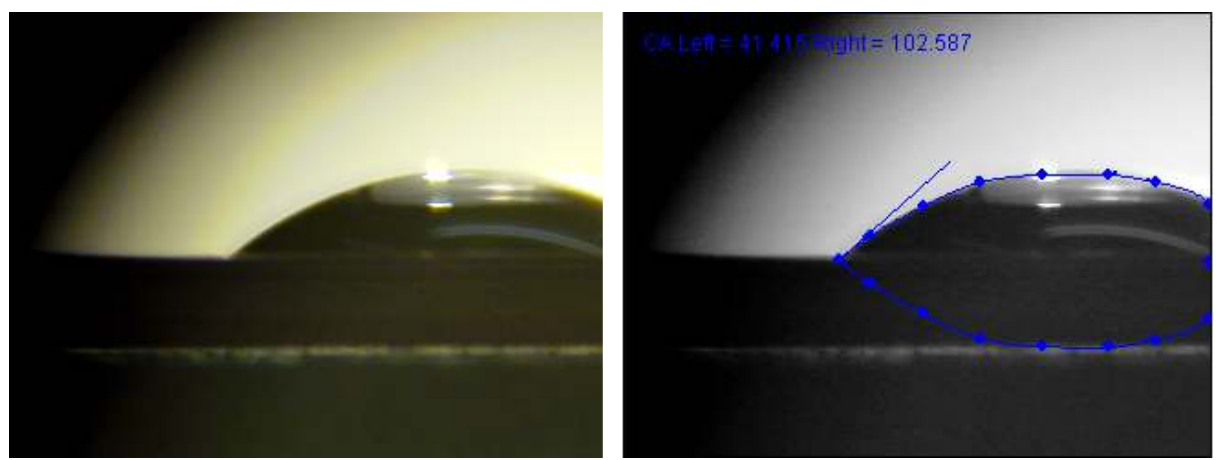

CA $\sim 41$

Table 5-3 NO Annealing

\footnotetext{
- 'NO Annealing' are the as-prepared films immediately after solvent evaporation at $100 \mathrm{C}(5 \mathrm{~min})$
} 


\subsubsection{Hr Annealing}

Thickness: 159nm MSE: 9.4

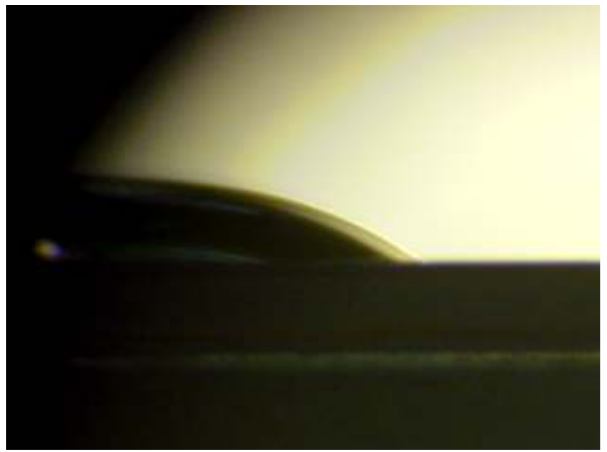

CA $\sim 32$

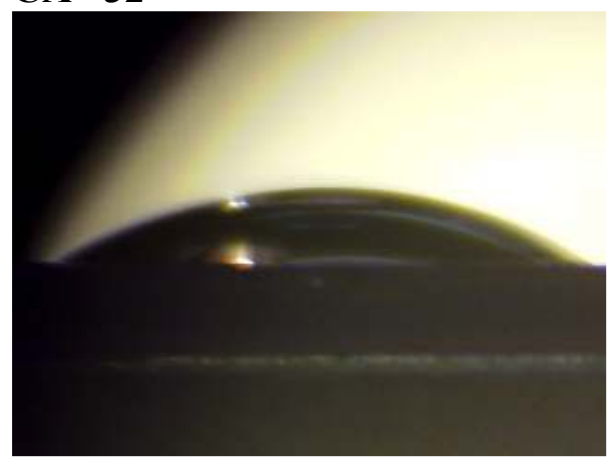

CA $\sim 31$

Table 5-4 12Hr Annealing

\subsubsection{Hr Annealing}

Thickness: 169nm MSE: 4.6

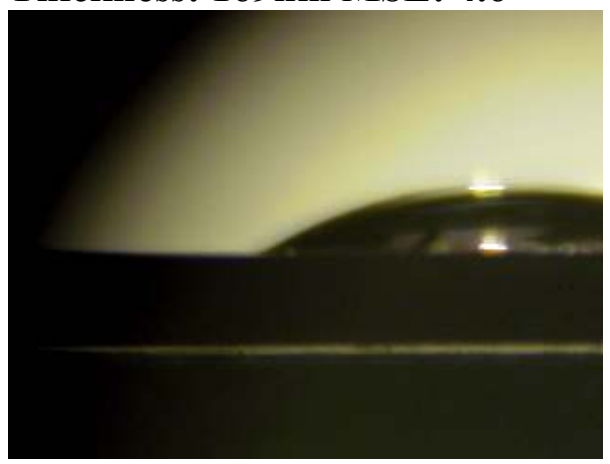

$\mathrm{CA} \sim 27$

Table 5-5 24Hr Annealing
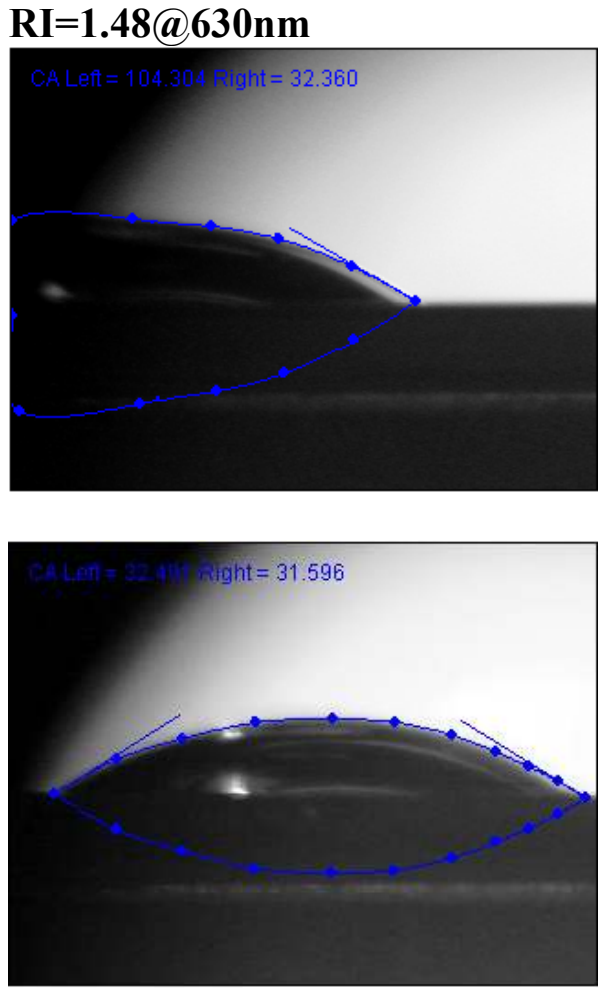

$\mathrm{RI}=1.47 @ 630 \mathrm{~nm}$

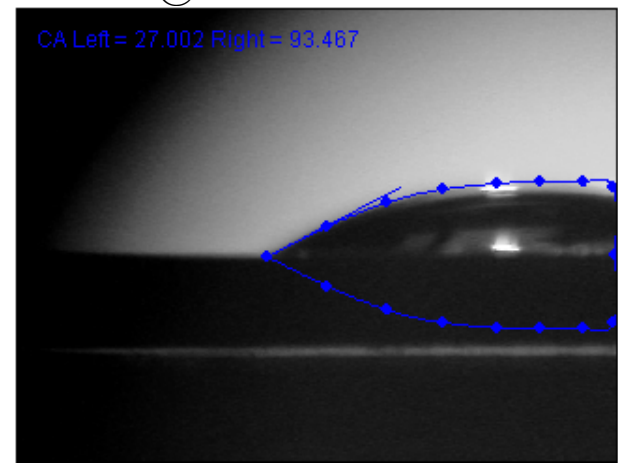




\subsection{Oxygen Plasma Treated Samples(the same above samples)}

The above samples were then Oxygen Plasma* treated for $1 \mathrm{~min}$ and immersed in cold DI water for $1 \mathrm{hr}$.

\subsubsection{NO Annealing*, 1min Oxy Plasma* treated samples}

Thickness: $160 \mathrm{~nm}$ MSE: 14.4

\section{RI=1.49@630nm}
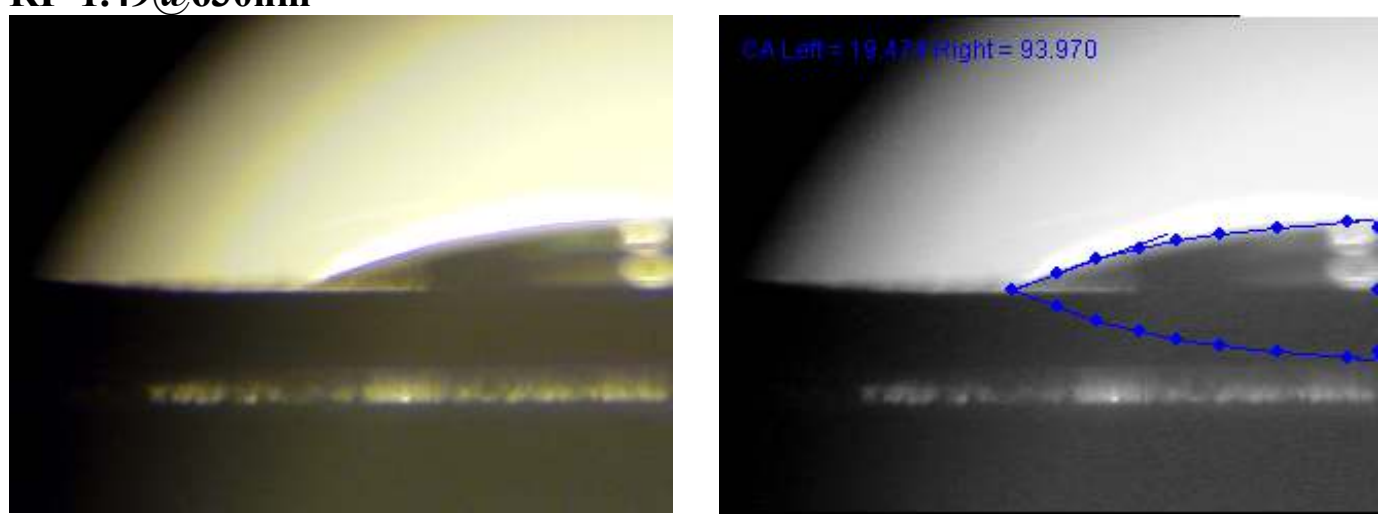

CA $\sim 19$
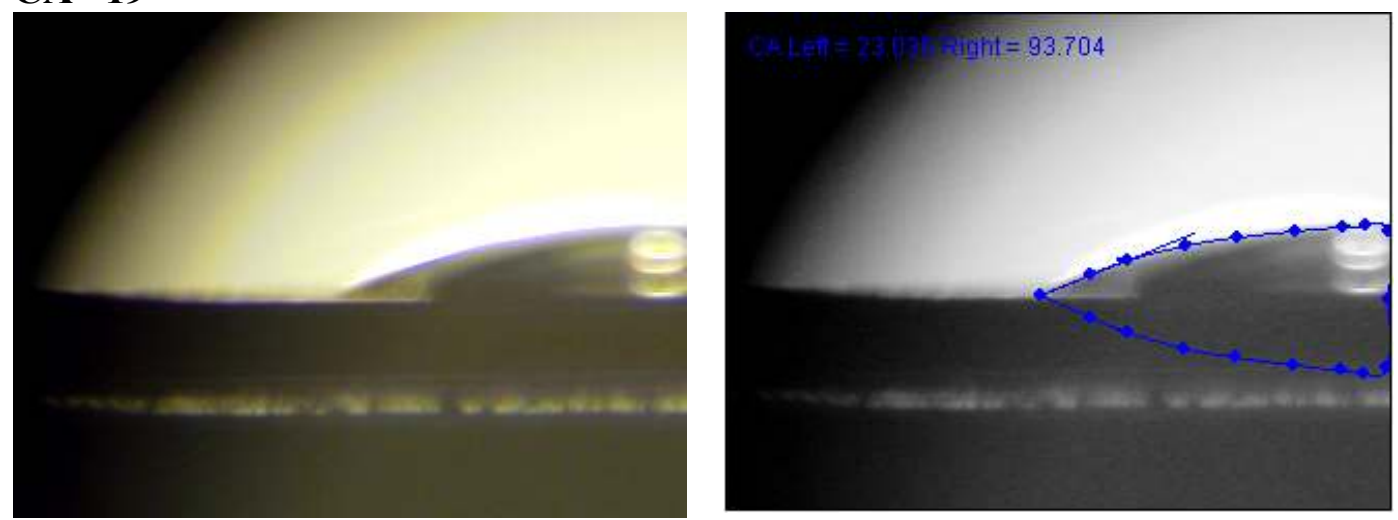

CA $\sim 23$

Table 5-6 NO Annealing, 1 min Oxy Plasma treated samples

- 'NO Annealing' are the as-prepared films immediately after solvent evaporation at $100 \mathrm{C}(5 \mathrm{~min})$

${ }^{*}$ Conditions of Oxygen Plasma cited in experimental section in chapter 4 


\subsubsection{Hr Annealing 1min Oxy Plasma* treated samples}

Thickness: 159nm MSE: 9.4

\section{RI=1.48@630nm}
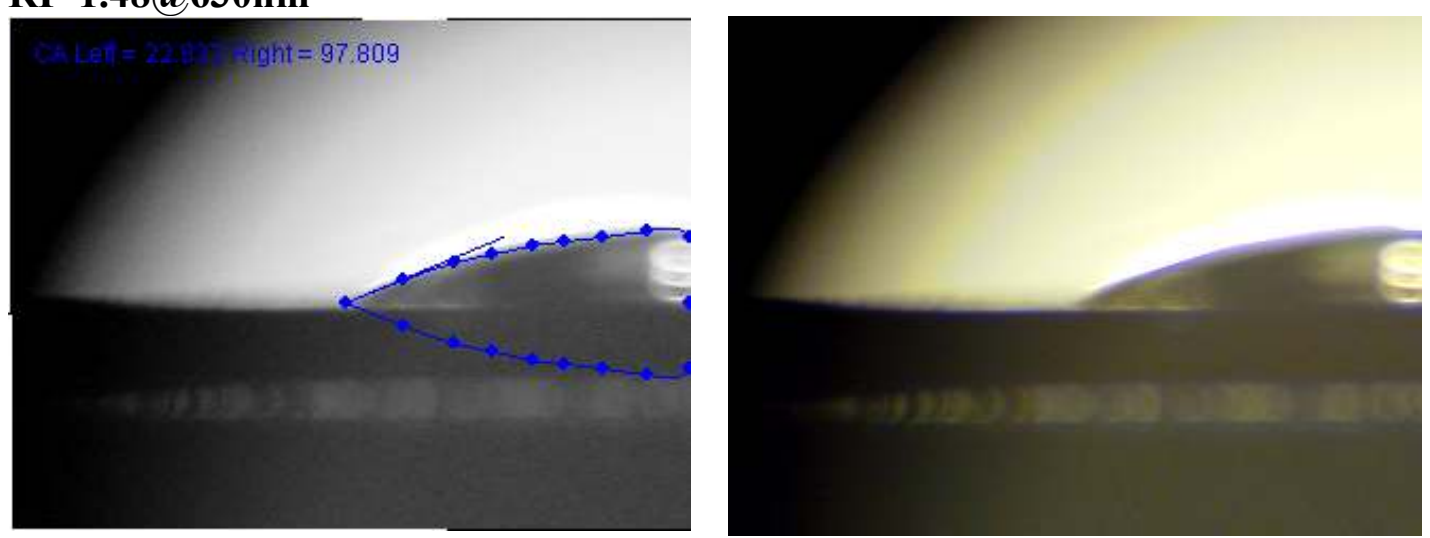

CA $\sim 23$
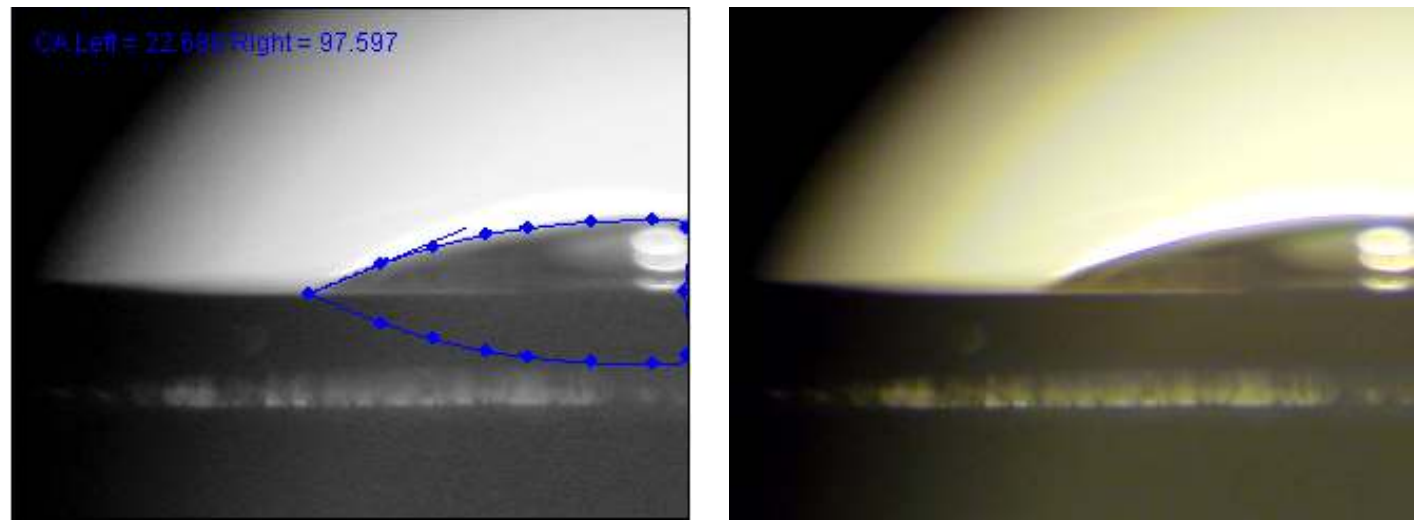

CA $\sim 23$

Table 5-7 12Hr Annealing 1min Oxy Plasma treated samples

\footnotetext{
${ }^{*}$ Conditions of Oxygen Plasma cited in experimental section in chapter 4
} 


\subsubsection{Hr Annealing 1min Oxy Plasma treated samples}

Thickness: 169nm MSE: 4.6

\section{$\mathrm{RI}=1.47 @ 630 \mathrm{~nm}$}
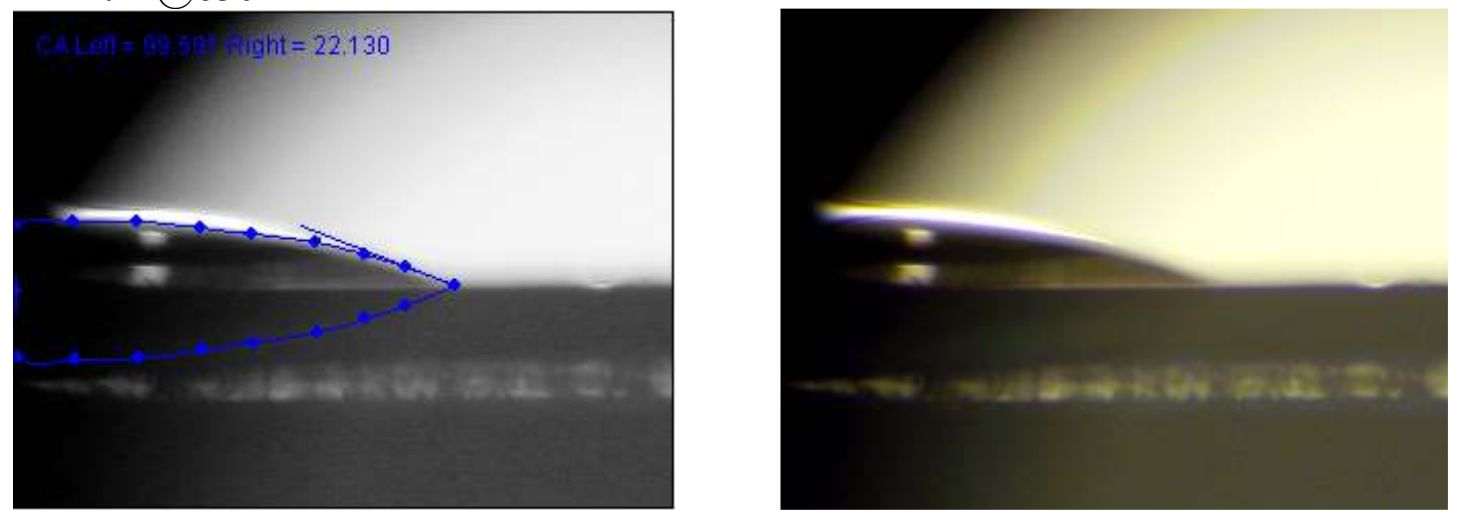

CA $\sim 22$
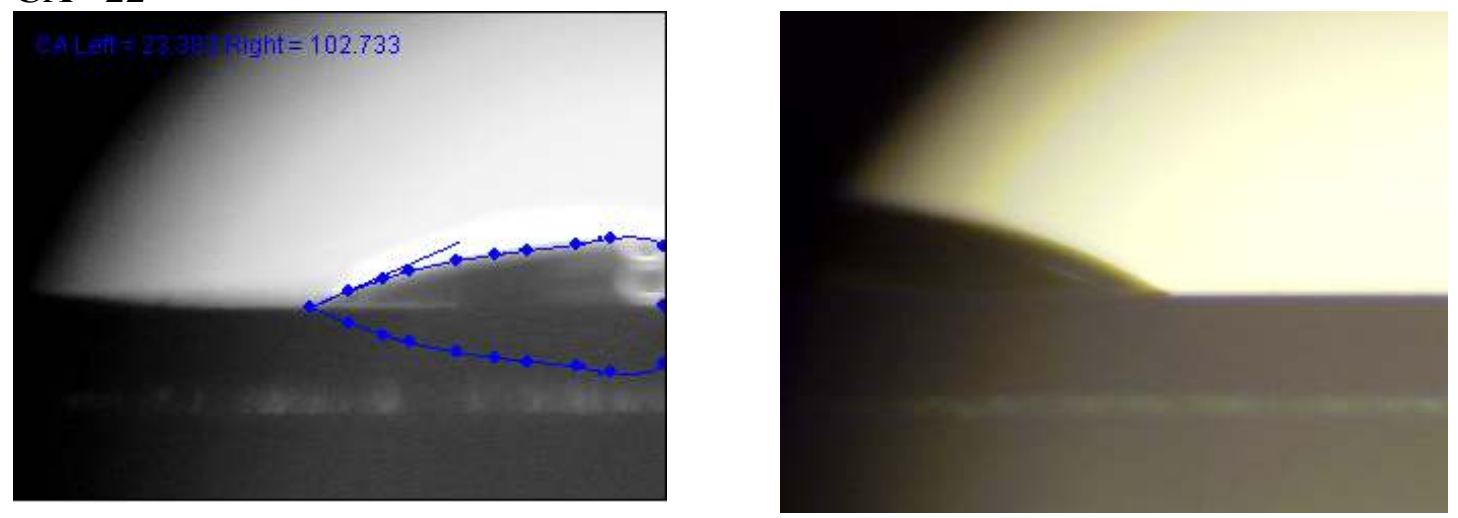

CA $\sim 23$

Table 5-8 24Hr Annealing 1min Oxy Plasma treated samples

As can be seen, the low temperature films that were Oxygen Plasma processed resulted in water contact angles that were comparatively lower than films that were not treated with an Oxygen plasma. An explanation for this has been presented in the discussion in section 5.5. 


\subsection{FTIR Characterizations}

The low temperature films were characterized using FTIR(Fourier transform infrared spectroscopy). The different FTIR plots of the films at different stages of the processing have been summarized below.

5.4.1 FTIR plots before Oxygen plasma treatment/water immersion and CTAB removal of low temperature processed films.

No Annealing(black) ${ }^{\bullet}$

$12 \mathrm{hr}$ Annealing at $150 \mathrm{C}(\mathrm{red})$

24hr Annealing at 150C(green)

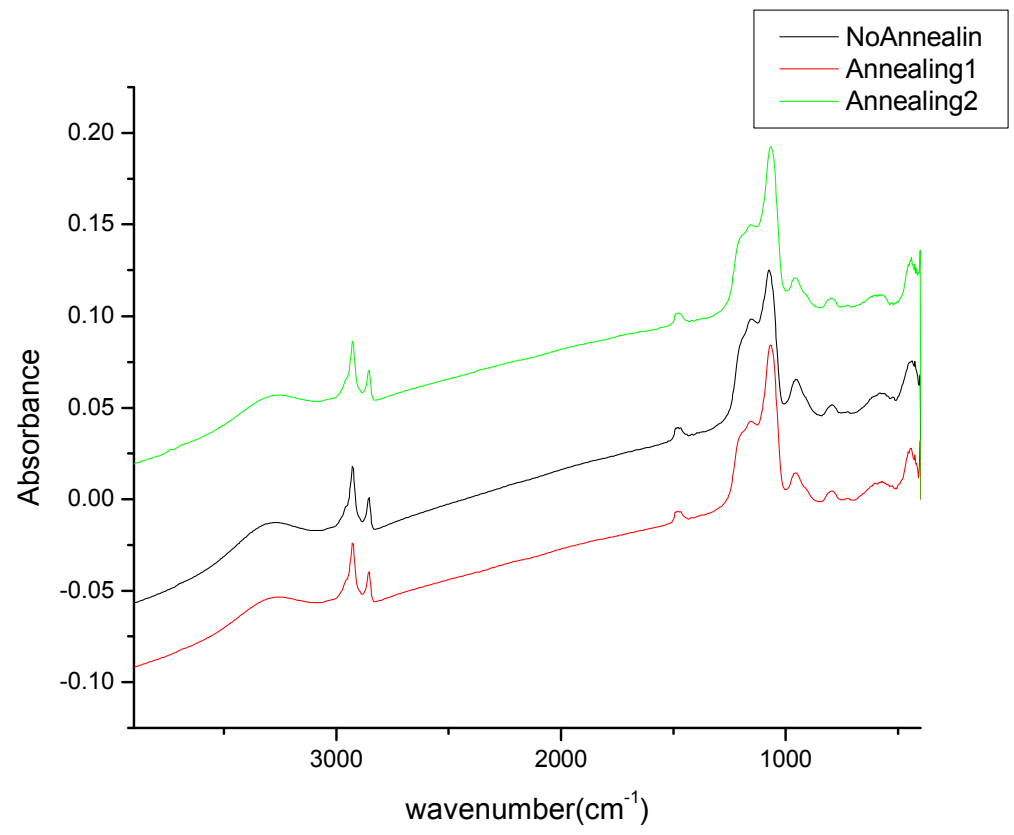

Figure 5-2 FTIR plots before Oxygen plasma treatment/water immersion and CTAB removal of low temperature processed films.

Presence of $\mathrm{CTAB}$ is seen as 2 small peaks which are the $\mathrm{C}-\mathrm{H}$ symmetric and antisymmetric stretching vibrations at 2850 and $2920 \mathrm{~cm}^{-1}$. Since the samples were not annealed at higher temperatures such as $450 \mathrm{C}$ at which all organic molecules disintegrate, $\mathrm{CTAB}$ is still preserved within the Siloxane network. The presence of CTAB is

\footnotetext{
- 'NO Annealing' are the as-prepared films immediately after solvent evaporation at $100 \mathrm{C}(5 \mathrm{~min})$
} 
undesirable. It renders the surface hydrophobic because of the presence of methyl groups which are hydrophobic.

\subsubsection{FTIR plots after Oxygen plasma treatment/water immersion but before CTAB} removal

No Annealing(black)

$12 \mathrm{hr}$ Annealing(red)

24hr Annealing(green)

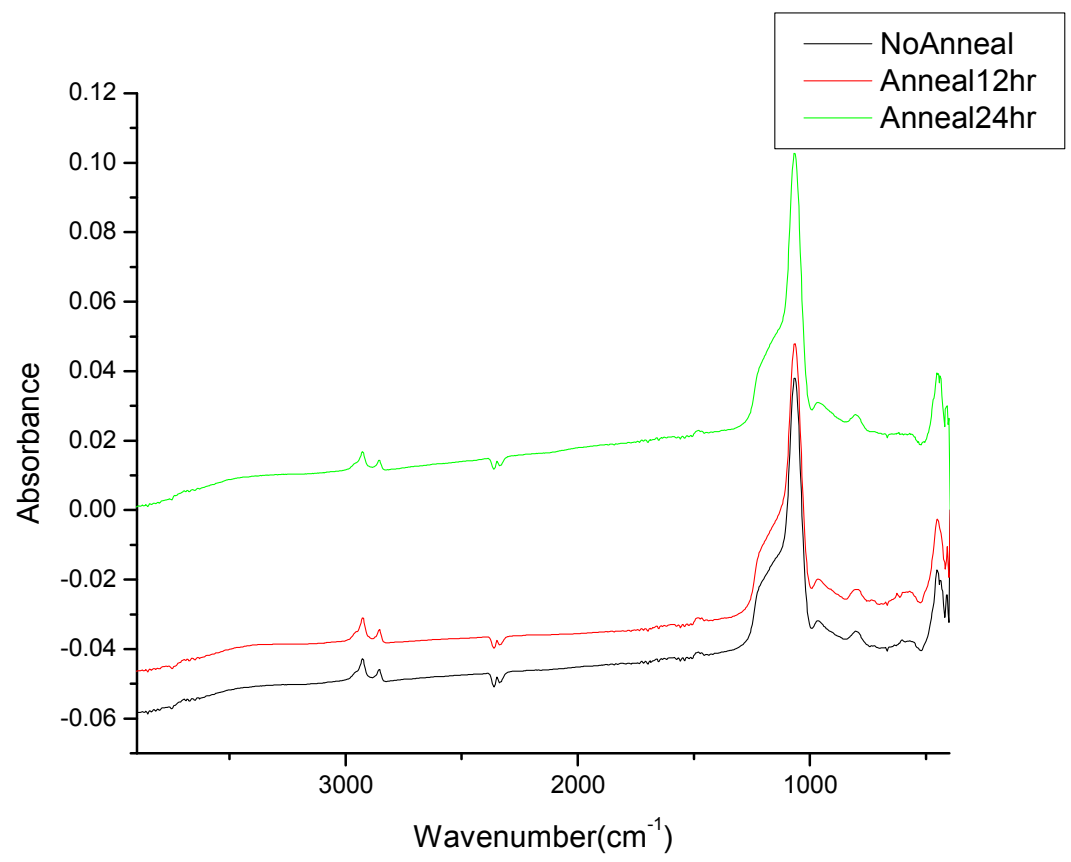

Figure 5-3 FTIR plots after Oxygen plasma treatment/water immersion but before CTAB removal.

As can be seen a mild Oxygen Plasma treatment with subsequent water immersion seems to reduce the presence of $\mathrm{CTAB}$ to a great extent. This is verified by the decrease in the peak height intensities at 2850 and $2920 \mathrm{~cm}^{-1}$.

- 'NO Annealing' are the as-prepared films immediately after solvent evaporation at $100 \mathrm{C}(5 \mathrm{~min})$ 
5.4.3 FTIR plots after CTAB removal WITHOUT Oxygen Plasma(By only exposing it to Ethanol vapours)

\section{No Annealing(black)}

$12 \mathrm{hr}$ Annealing(red)

24hr Annealing(green)

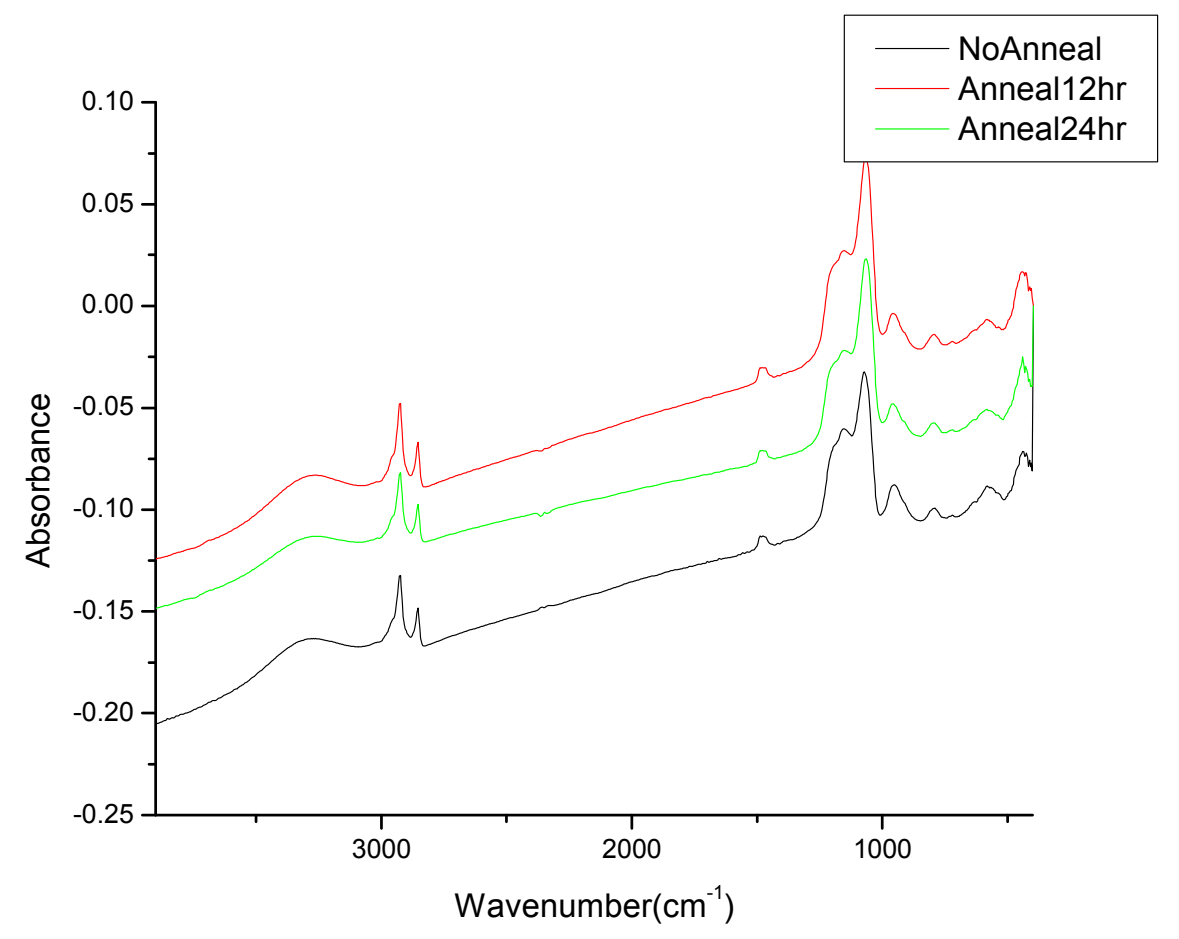

Figure 5-4 FTIR plots after CTAB removal WITHOUT Oxygen Plasma(By only exposing it to Ethanol vapors).

The CTAB removal by exposure to ethanol vapors was not very effective. The presence of CTAB is still seen in the FTIR plots. Ethanol fumes can diffuse through porous networks but the actual transport of surfactant molecules from within the film to the outside is made possible only with the ethanol washing as shown in the below FTIR plots. 


\subsubsection{FTIR plots after CTAB removal WITHOUT Oxygen Plasma (By exposing it to Ethanol vapors + washing with cold Ethanol)}

\section{No Annealing(black)}

$12 \mathrm{hr}$ Annealing(red)

24hr Annealing(green)

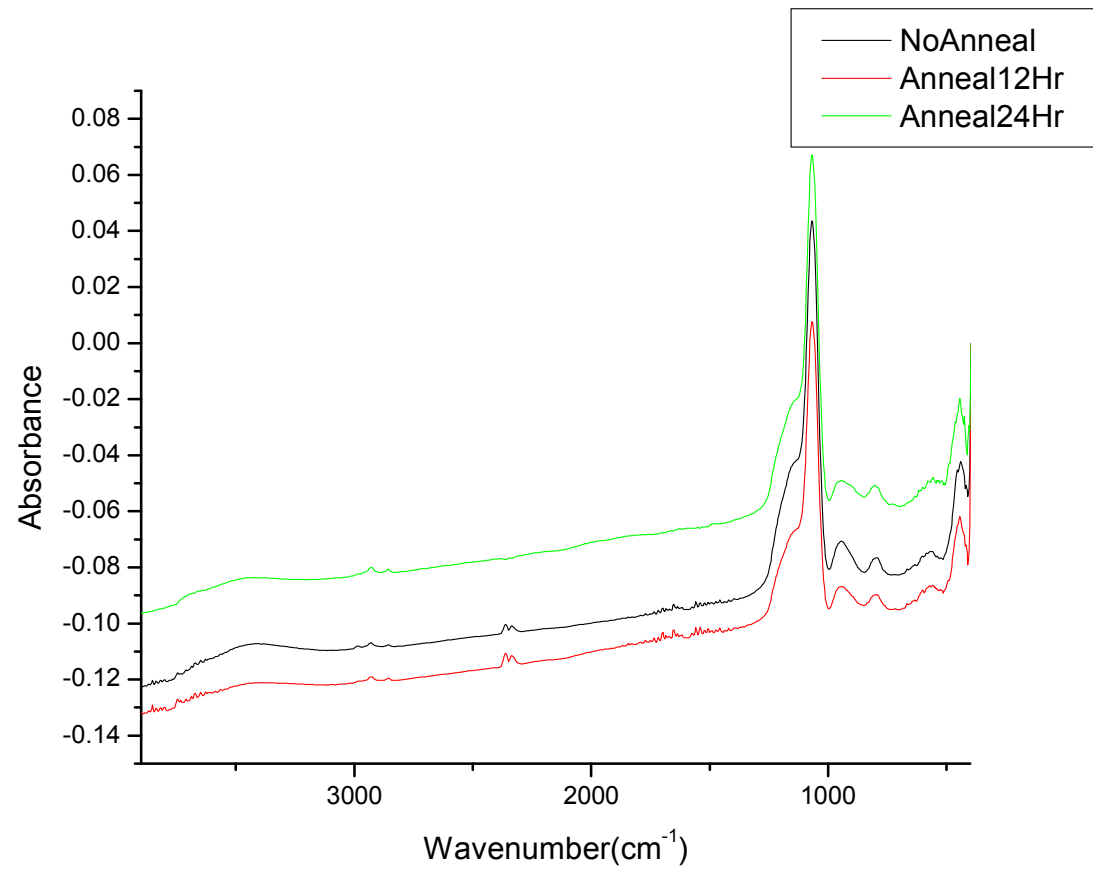

Figure 5-5 FTIR plots after CTAB removal WITHOUT Oxygen Plasma (By exposing it to Ethanol vapors + washing with cold Ethanol).

As can be seen in the above graphs, there almost seems to be no presence of the CTAB after the ethanol washing. This is a good thing in a way as no high temperature processing is required to burn off the $\mathrm{CTAB}$ within the film. The presence of pores in a low temperature process could not be confirmed as spectroscopic ellipsometry was not performed on these films. Nothing can be said about the porosity without this data. 


\subsection{Discussion on the effect of mild Oxygen Plasma}

Organic components such as surfactant molecules and un-hydrolized ethoxide molecules disintegrate[58] when the sol-gel films are exposed to a mild Oxygen plasma. One of the reasons that can be cited for the decrease in contact angles after the mild Oxygen plasma on the high-temperature-processed films is that [58] the atomic oxygen generated by Oxygen plasma reacts with hydrocarbon molecules through $\mathrm{H}$-atom abstraction and the resulting $\mathrm{OH}$ radicals induce formation of surface silanols so that high concentrations of silanol are incorporated into silica films. This causes a further decrease in the contact angles due to these $-\mathrm{OH}$ groups. The Si-OH bending band at around $960 \mathrm{~cm}^{-1}$ appears for all samples in the above FTIR plots but their relative intensities are much different which is dependent on the degree of cross-linking of the film. A higher cross-linked film would have lesser $\mathrm{Si}-\mathrm{OH}$ moieties and hence a lesser peak intensity at $960 \mathrm{~cm}^{-1}$. As can be seen in Fig 5-15 thru 5-18, the peak at $1085 \mathrm{~cm}^{-1}$ represents the pure stretching vibration of SiO-Si bonds, and the peak at around $950 \mathrm{~cm}^{-1}$ is induced by the $-\mathrm{OH}$ groups and assigned to the stretching vibration of Si-OH bonds. It can be seen that the $-\mathrm{OH}$ peak at $950 \mathrm{~cm}^{-1}$ in Fig 5-16 has a much smaller intensity as compared to the peak in Fig 5-15. Also the relative peak intensity $\mathrm{Si}-\mathrm{O}-\mathrm{Si} / \mathrm{Si}-\mathrm{OH}$ ratio, is much higher in Fig 5-16 as compared to

Fig 5-15. This could be due the additional cross-linking of the Si-O-Si network due to Si$\mathrm{OH}$ condensation induced as a result of the Oxygen plasma. 


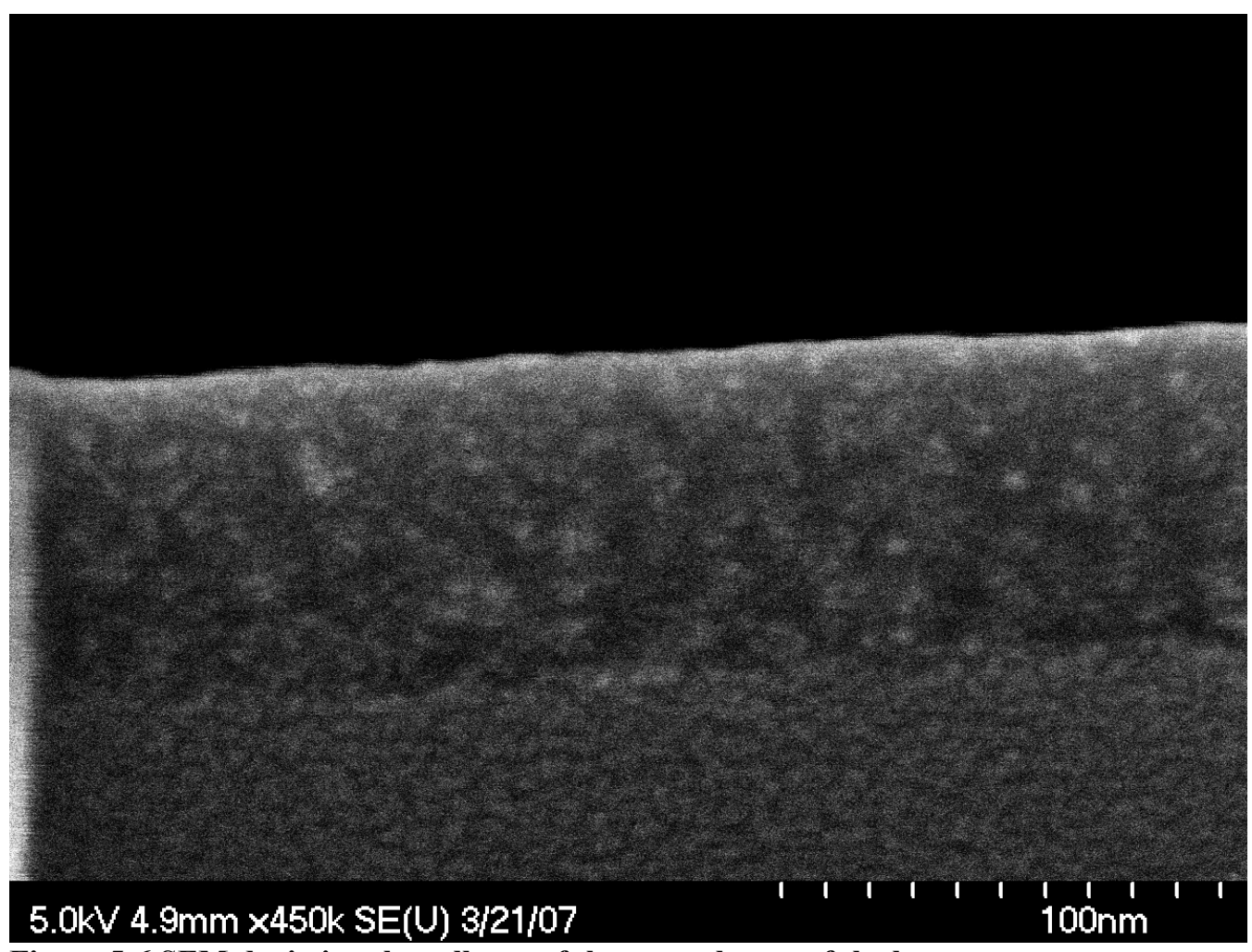

Figure 5-6 SEM depicting the collapse of the upper layers of the low temperature processed films due to Oxygen Plasma

Figure 5-7 is one of the SEM's that was obtained on a low-temperature-processed, oxygen plasma exposed sol-gel film which is the same as Figure 5-2 at higher magnification. As can be seen, there appears to be two layers, the topmost layer extending to a depth of less than $100 \mathrm{~nm}$. The reason for the difference in the structural changes can be attributed to the effect of the mild oxygen plasma on the sol-gel film. The damage becomes much more prominent in the low temperature case as the network has not yet fully cross-linked. The plasma results in the collapse of the structure for the first few tens of nanometers.

With not much success in developing a low-temperature process for effectively crosslinking the silica matrix and effectively eliminating the templating agents, it was decided to explore the hydrophobic approach in order to create super-hydrophobic surfaces. One 
of the easiest ways in developing super-hydrophobic surfaces would be to use a low surface energy material with appropriate surface roughness. Since nano-particles could be used to induce surface roughness, it was decided to synthesize nano-particles prepared in the lab. 


\subsection{Hydrophobic Approach: Nano-Materials synthesis}

At some point during the research, it was decided to adopt the super-hydrophobic approach. As mentioned earlier, one of the requirements for the fabrication of the superhydrophobic surfaces was appropriate surface roughness. Surface roughness could be induced either through porosity or building blocks such as nano-materials. For this we decided to synthesize our own nano-materials.

5.6.1 Organo-Silicate Nanorods: The organo-silicate(PMSSQ) nano-rods were synthesized according to the procedure as elaborated in the Experimental section. The TEM images have been shown below
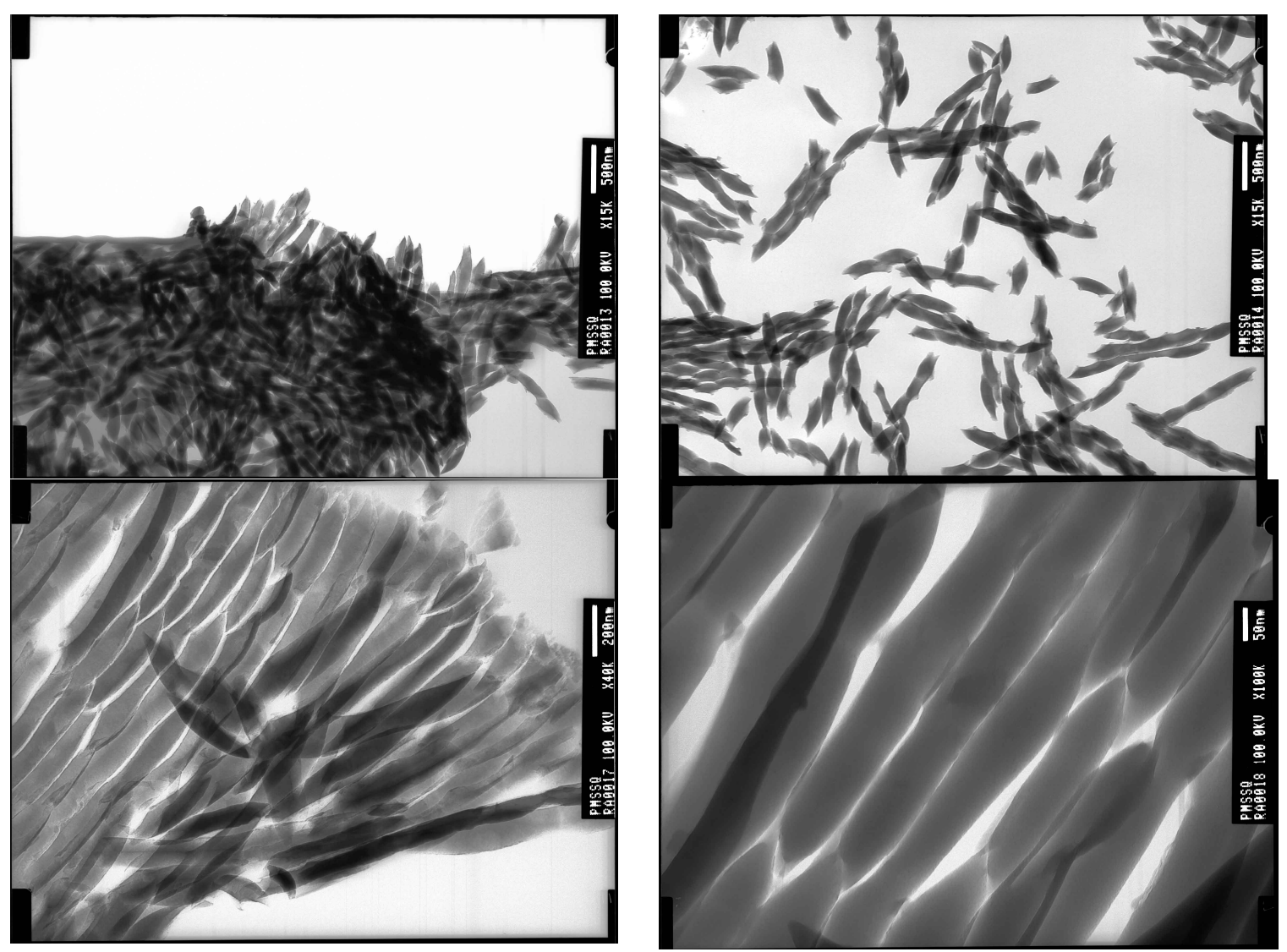

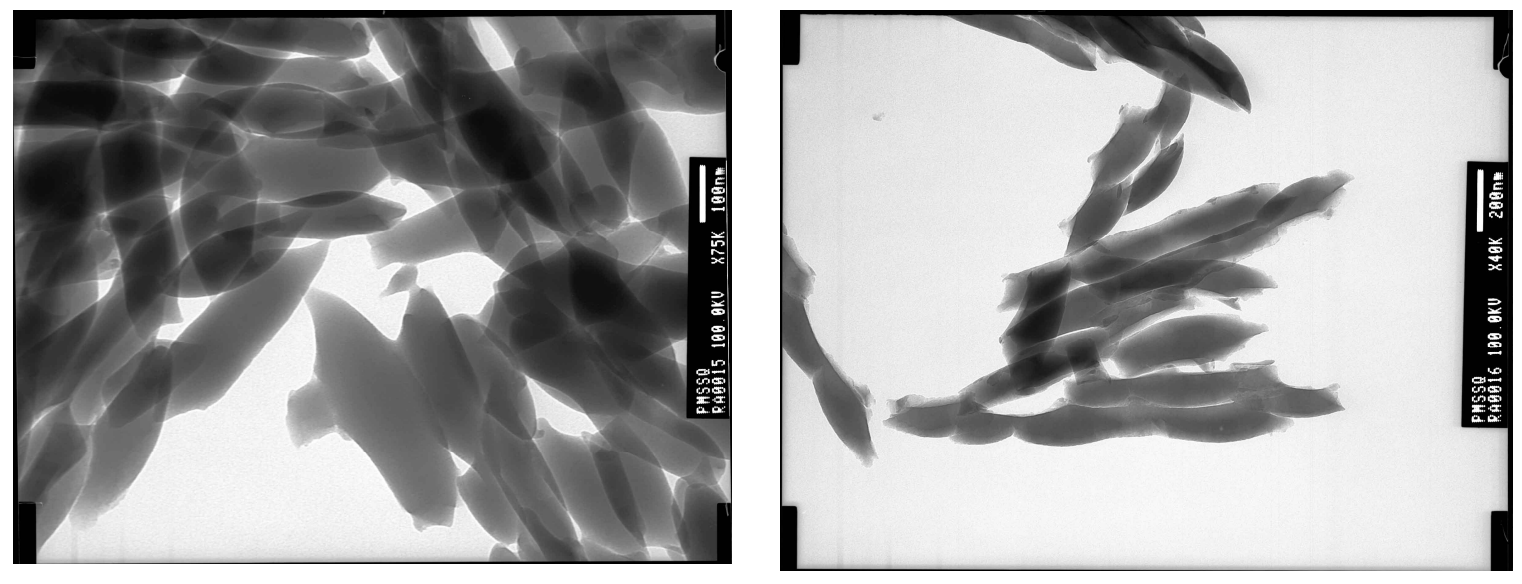

Figure 5-7 PMSSQ(poly(methylsilsesquioxane)) Nano-rods

TEM imaging revealed that the nano-rods are seen to possess widths in the $50-100 \mathrm{~nm}$ range with lengths in the range of 500nm giving it an aspect ratio between 50-10. 


\subsubsection{Non-porous Silica sol-gel films: Loading with organo-silicate Nano-rods}

The obtained films after loading the Sol-gel solution with the nano-rods were transparent but the contact angles obtained with water were no better than the control samples(the sol-gel films without the nano-rods). As earlier mentioned in the Experimental section, the sol-gel solutions were loaded at $0.5 \mathrm{wt} \%, 1 \mathrm{wt} \%, 2.5 \mathrm{wt} \%$, and $5 \mathrm{wt} \%$ and the corresponding films were characterized by contact angle measurements. It was seen that at higher loadings such as $2.5 \mathrm{wt} \%$ and $5 \mathrm{wt} \%$, the contact angle were seen to decrease from 80degrees to as low as 60degrees.

\section{RESULTS}

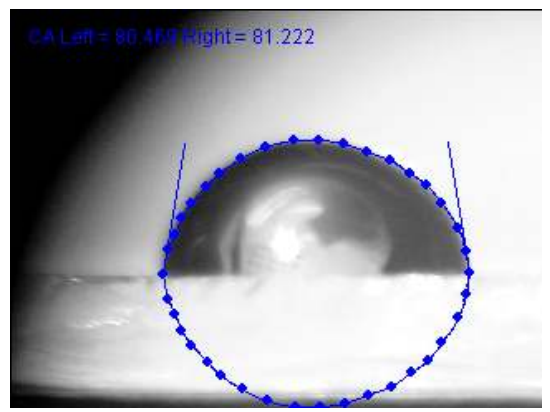

Sol-gel Ctrl \#1 (0wt\% particles loaded)

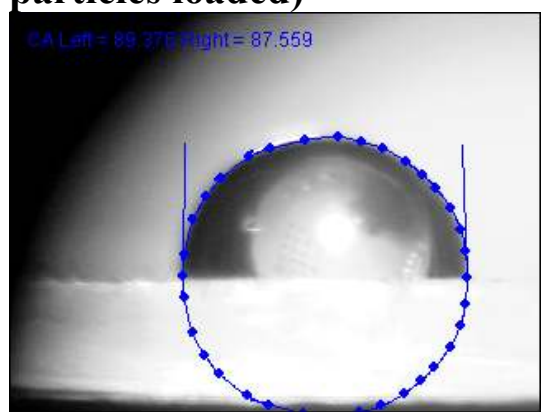

Sol-gel 0.5\% \#1 (0.5wt\% Organo-silicate particles loaded)

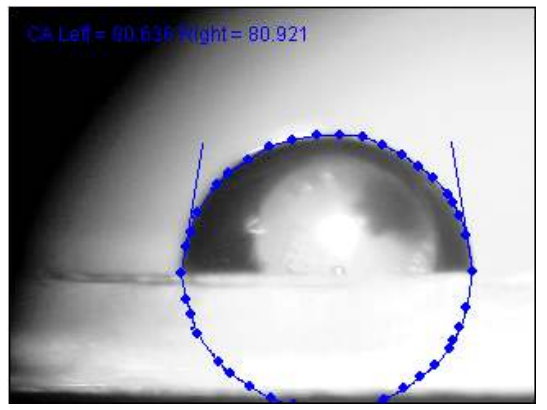

Sol-gel Ctrl \#2 (0wt\% particles loaded)

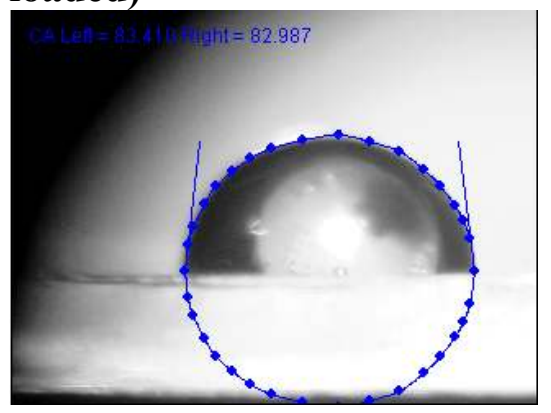

Sol-gel 0.5\% \#2 (0.5wt\% Organosilicate particles loaded) 


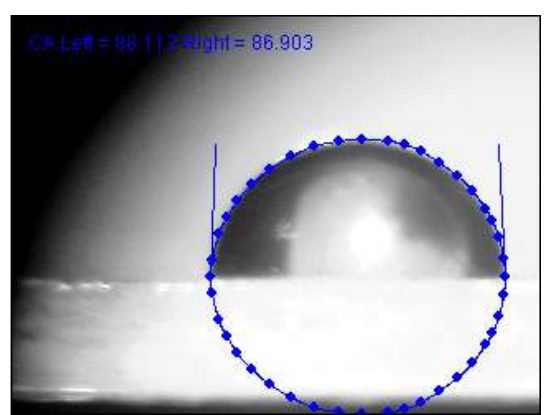

Sol-gel 1\% \#1 (1wt\% Organosilicate particles loaded)

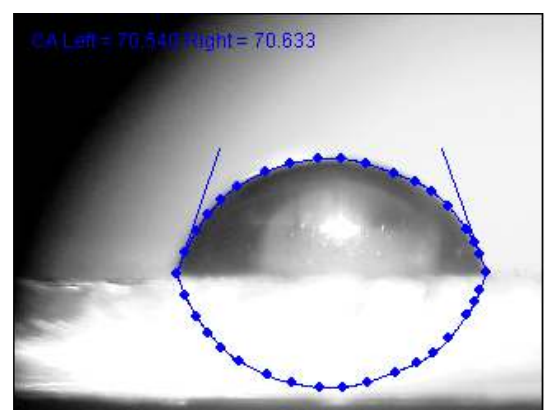

Sol-gel 2.5\% \#1 (2.5wt\%

Organo-silicate particles loaded)

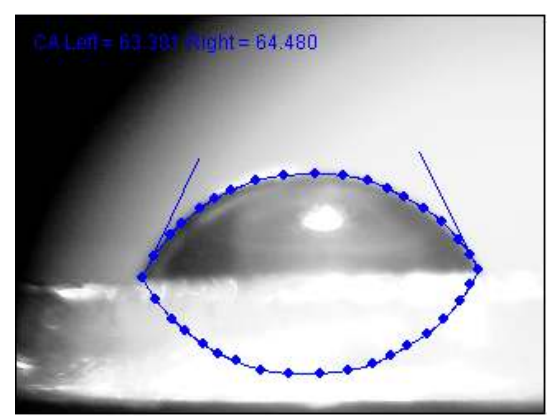

Sol-gel 5\% \#1 (5wt\% Organosilicate particles loaded)

Table 5-9 CA Images of sol-gel films loaded with organo-silicate Nano-rods

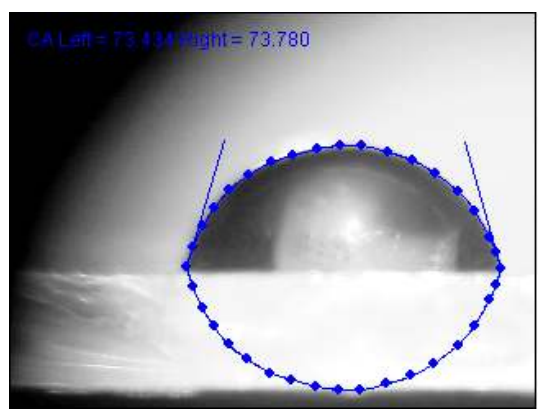

Sol-gel 1\% \#2 (1wt\% Organosilicate particles loaded)

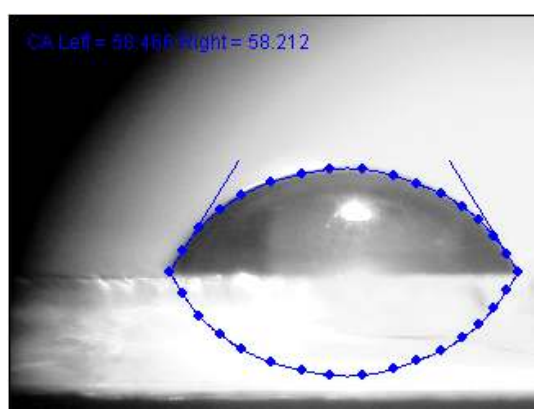

Sol-gel 2.5\% \#2 (2.5wt\% Organosilicate particles loaded)

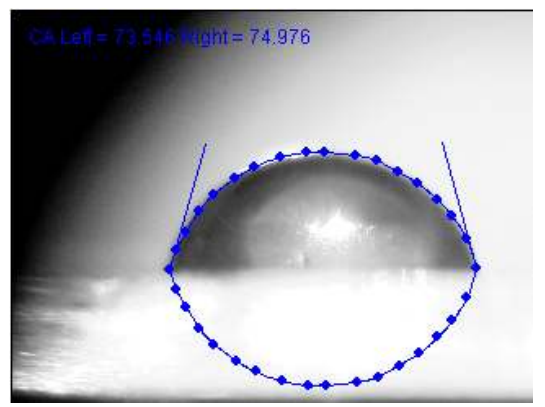

Sol-gel 5\% \#2 (5wt\% Organosilicate particles loaded)

The same films after the HMDS treatment resulted in the films attaining contact angles of around 90degrees. 


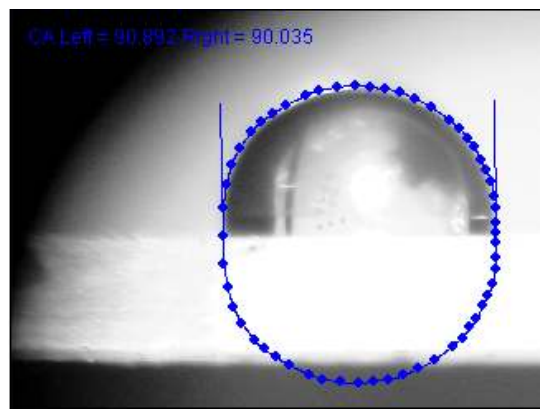

Sol-gel film with $0.5 w t \%$ organo-silicate particles

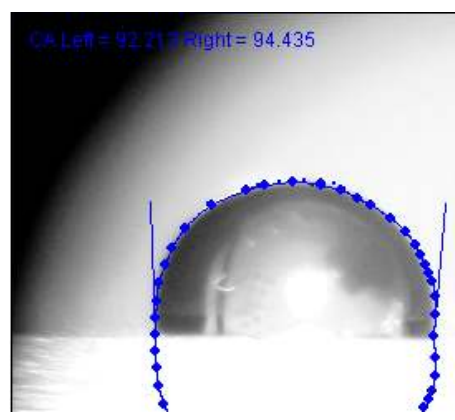

Sol-gel film with $2.5 w t \%$ organo-silicate particles

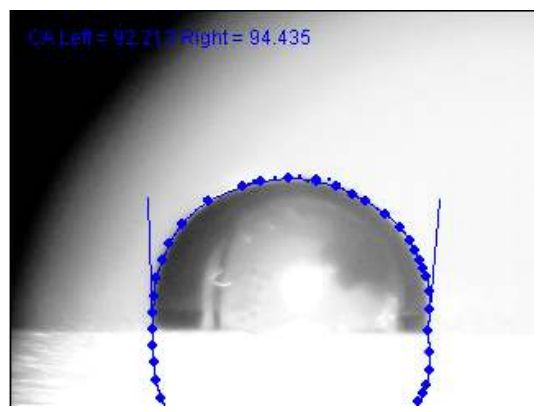

Sol-gel film with $1 \mathrm{wt} \%$ organosilicate particles

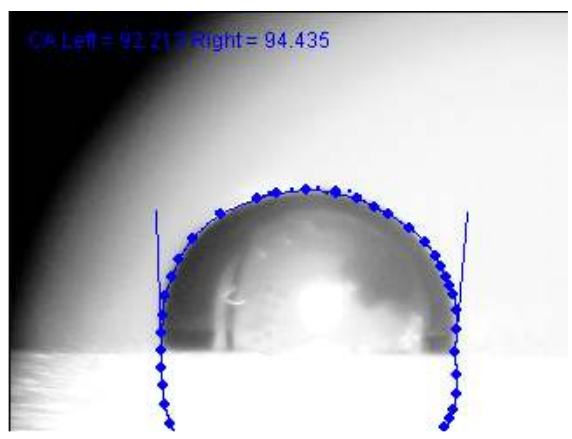

Sol-gel film with $5 w t \%$ organosilicate particles

Table 5-10 CA Images of Sol-gel thin films loaded with nano-rods

\subsubsection{Discussion on nano-rod loaded sol-gel films}

Loading nano-rods into a sol-gel matrix is indeed a good way by which roughness can be induced but the bigger challenge in getting the nano-rods to align vertically is a major setback. The roughness of a surface is enhanced several fold when the nano-rods are aligned vertically as compared to being aligned horizontally. Modeling results have shown that the nano-pillar configuration[59] is one of the best configurations up to a certain inter-pillar distance wherein the water droplet is seen to reside on the tips or the top surfaces of these nano-pillars. Since the interfacial area between the droplet and the surface is very small in a vertically aligned nano-pillar configuration, the predicted contact angle by the Cassie-Baxter theory is expected to be very large. The nano-rods that 
were loaded in the sol-gel films were most likely aligned horizontally in the silica matrix and oriented in random directions causing no significant surface roughness on the film surface. No further characterizations such as AFM and SEM were carried out on the films to observe film morphology. 


\subsection{TEFLON THIN FILMS}

\subsubsection{Porous polymer films using micro-emulsions}

The porous Teflon films that were made using the micro-emulsion process were characterized using optical microscopy. The images have been shown below.
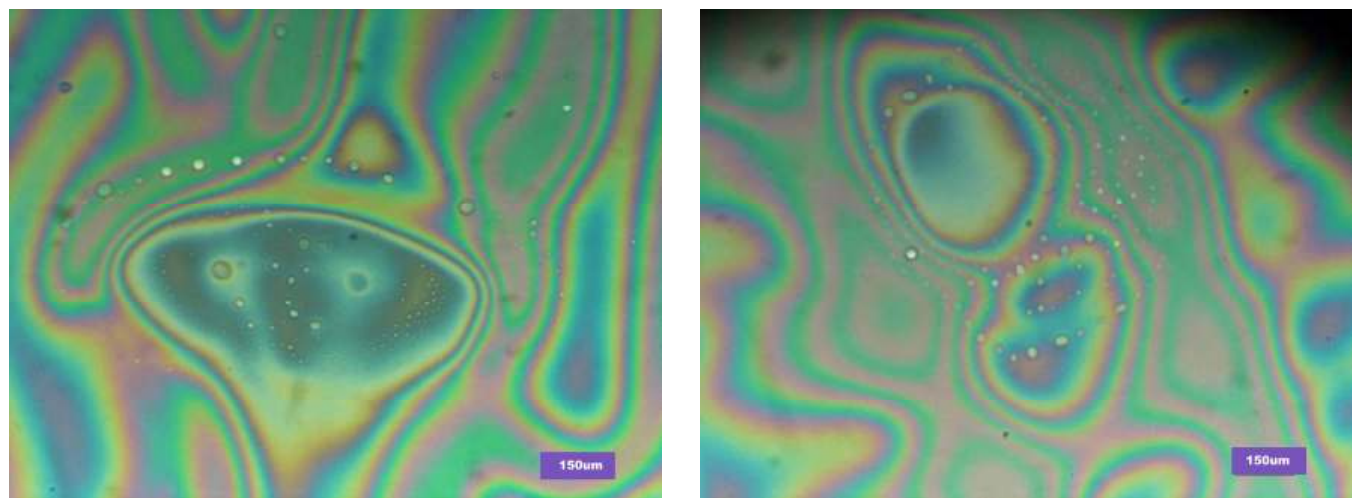

Table 5- 11 Teflon Films using ethanol Micro-emulsions - characterized using optical microscopy

The above two pictures were taken using Iso-propanol as the immiscible solvent. Isopropanol lead to better pore formation but the over-all pore formation was below

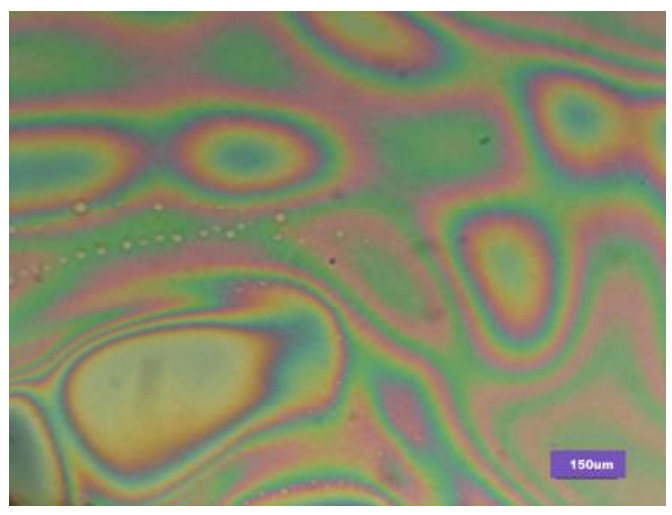
satisfactory as the pores occurred in far placed clusters throughout the film. They were not evenly distributed across the film. There is a possibility that the pores created could be in the nanometer range, hence not visible by an optical microscope.

Figure 5-8: Image of Teflon micro-emulsion films using Ethanol as the non-solvent.

The image in Fig 5-22 is taken using Ethanol as the non-solvent for the micro-emulsion process. Different films were made using Ethanol varying the ethanol wt $\%$ in each of the films. As earlier mentioned the films were characterized using optical microscopy and 
contact angle measurements. Below are the contact angles with respect to different ethanol wt $\%$.
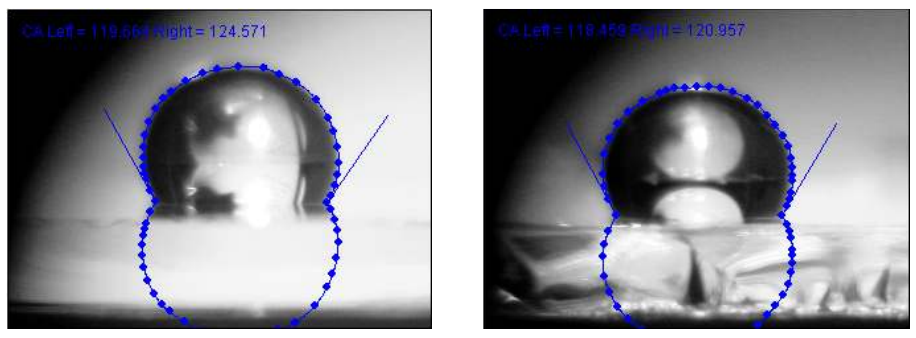

Teflon with $1 \%$ ethanol loading

\section{Teflon with $3 \%$ ethanol} loading

CA 119 124

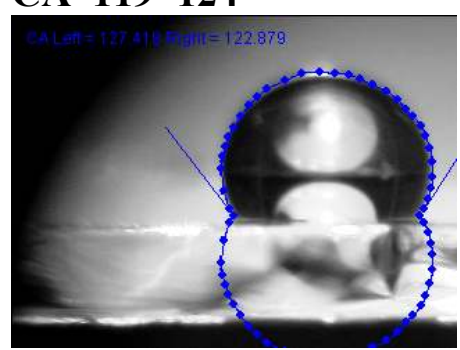

CA 118 120

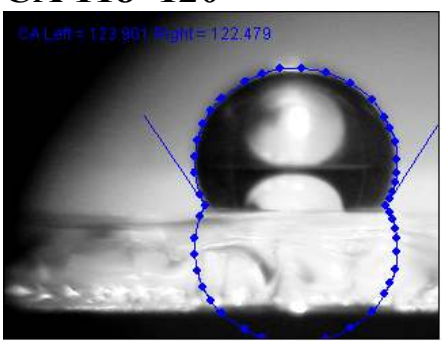

Teflon with $5 \%$ ethanol loading

Teflon with $10 \%$ ethanol loading.

CA 127 123

CA 124 122

Table 5-12 Images of contact angles of Teflon thin films using micro-emulsions

As can be seen these pores created with the ethanol micro-emulsion have a very small effect on the CA's(contact angles) due to the fact that the micron-sized pores occur only in clusters and are not uniformly distributed. 

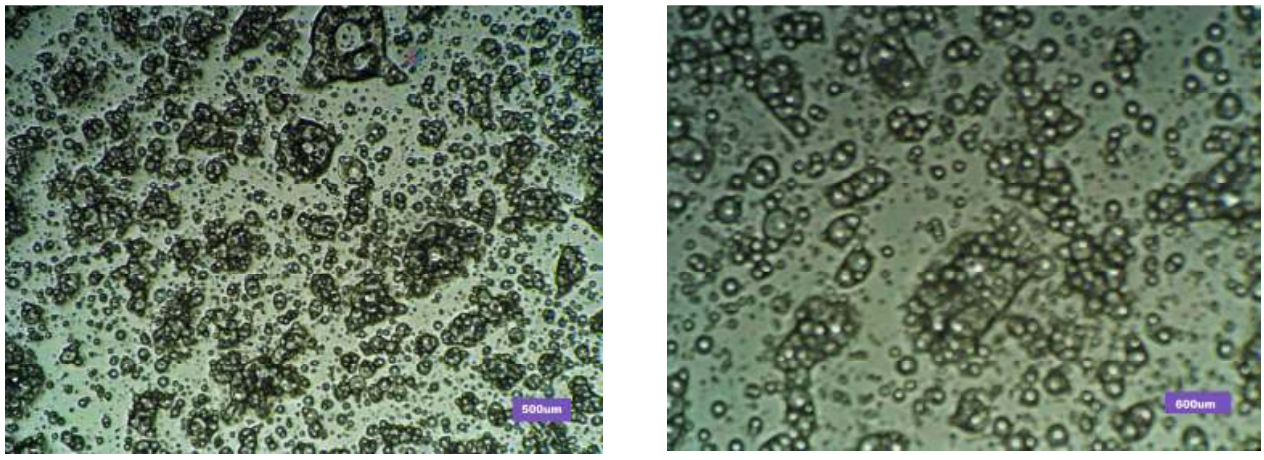

\section{Images of Porous Teflon at Images of Porous Teflon at magnifications of $5 x$ magnifications of $10 x$}

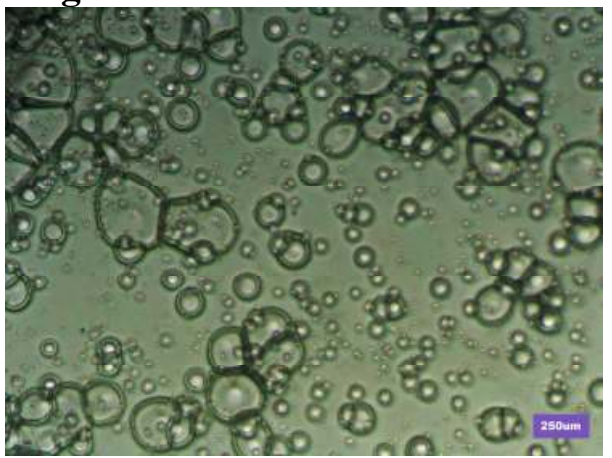

\section{Images of Porous Teflon at Images of Porous Teflon at magnifications of $20 x$ magnifications of $40 x$}

Table 5-13 Images of water based micro-emulsions Teflon films

In the images shown above, water was used as the non-solvent along with the Teflon solution to make the micro-emulsion. As shown above, the micro-emulsions that were made using ${ }^{1} \mathrm{MEK}$ (methyl-Ethyl ketone), Ethanol, Iso-propanol as the non-solvents, resulted in pore formation that could not be characterized with optical microscopes even with magnifications up to 40x. This could possibly be because of the formation of the pores in the nanometer ranges. The only pores that could be seen using optical microscopy occurred in random clusters throughout the film as shown in the results above.

\footnotetext{
${ }^{1}$ Results have not been shown here
} 
As can also be seen in the above images, the pores developed are very large in the range of tens of micrometers. It was observed that the size of the pores could be controlled by controlling the degree of mixing of the two immiscible solutions and the amount of immiscible solvent used.

Only when such micron sized pores are created uniformly across the entire surface, the contact angles of water droplets are expected to increase as a result of the uniform roughness induced across the film, making the films more hydrophobic.

\subsubsection{Transmission Spectra}

Below are the UV-VIS transmission spectra of the different films that were created using ethanol for the micro-emulsion.

\section{Transmittance Plots}

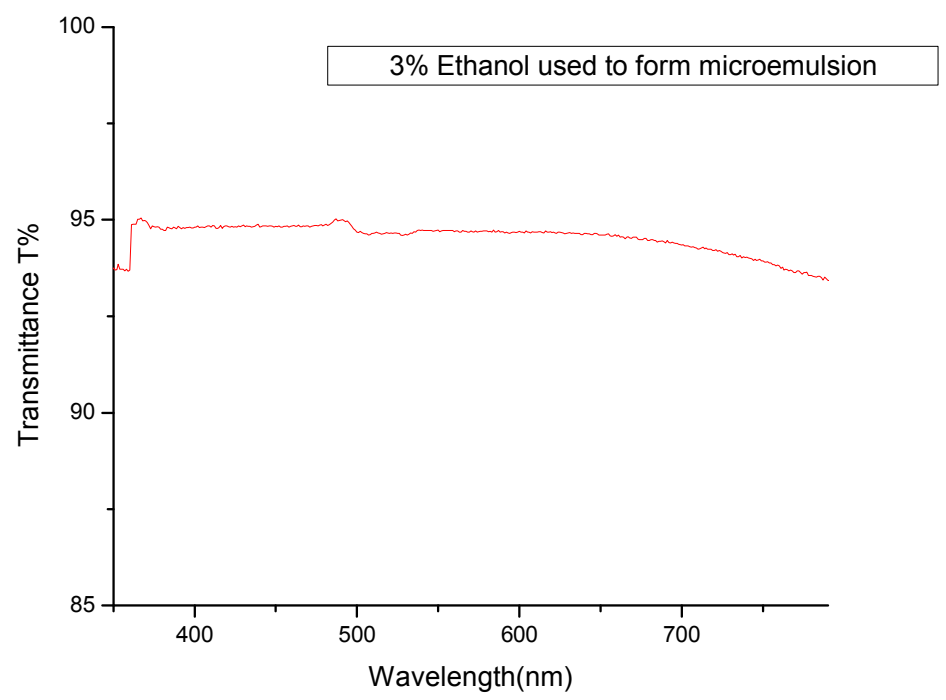

Figure 5-9 UV-VIS transmission spectra of $3 \%$ ethanol based micro-emulsion films 


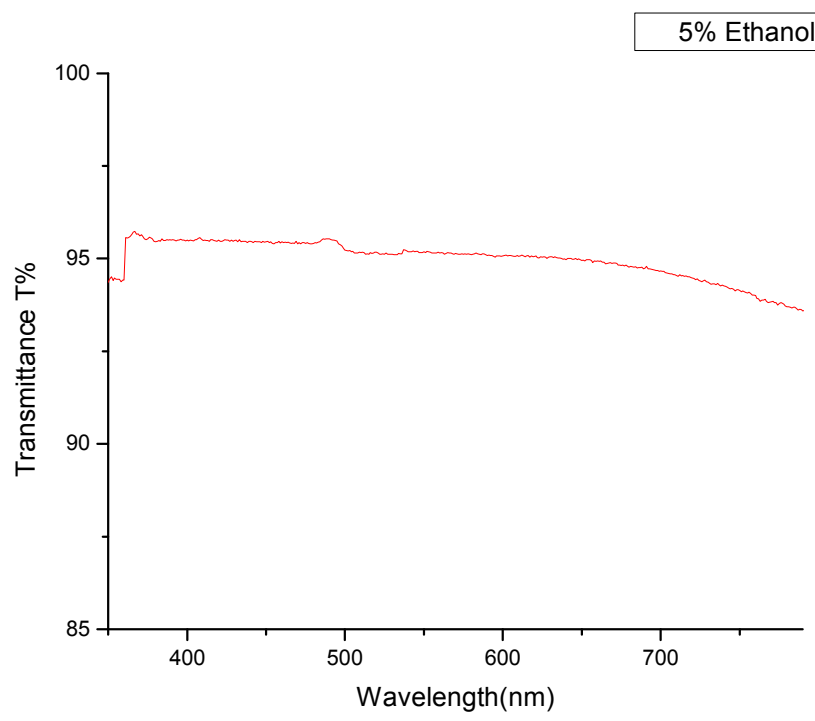

Figure 5-10 UV-VIS transmission spectra of 5\% ethanol based micro-emulsion films

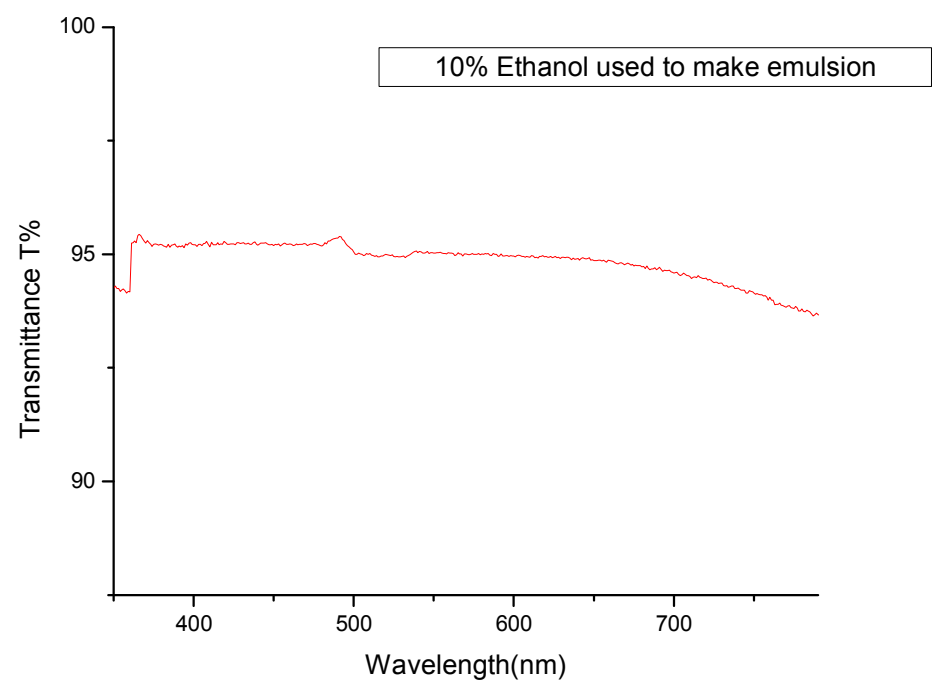

Figure 5-11 UV-VIS transmission spectra of $10 \%$ ethanol based micro-emulsion films

As can be seen in the above two UV-VIS transmission spectra, transmission was in excess of $90 \%$ which made these films excellent in terms of being transparent. The contact angles of the above films did not differ significantly from the control samples(smooth Teflon films without any micro-emulsion induced roughness) 


\subsubsection{Porous Teflon using PPG \& PGMEA}

In another experiment, Porous Teflon films using PPG and PGMEA were added to the Teflon solution to form micro-emulsions. The films were characterized using optical microscopy.

Table 5-14 displays the images of the different films that were created using PPG and PGMEA as the sacrificial templates. 


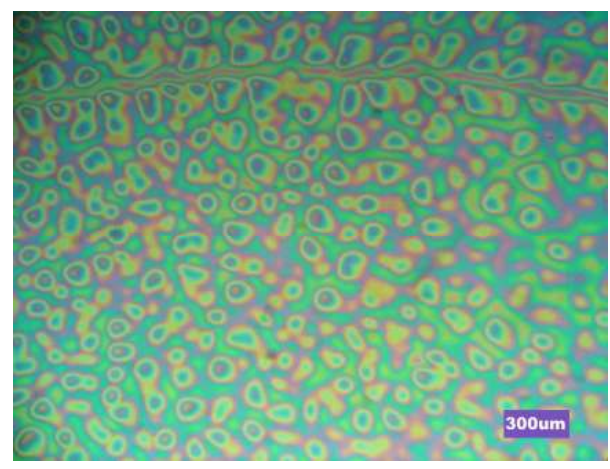

Plain Teflon without any PPG or PGMEA - CTRL

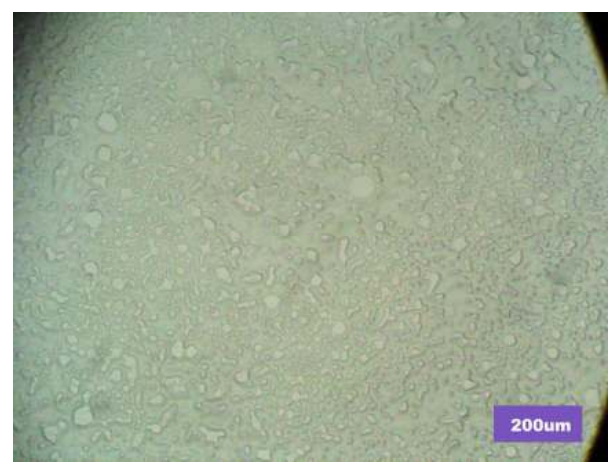

3\% Teflon:PGMEA:PPG = 3:1.5:0.75 at 40x

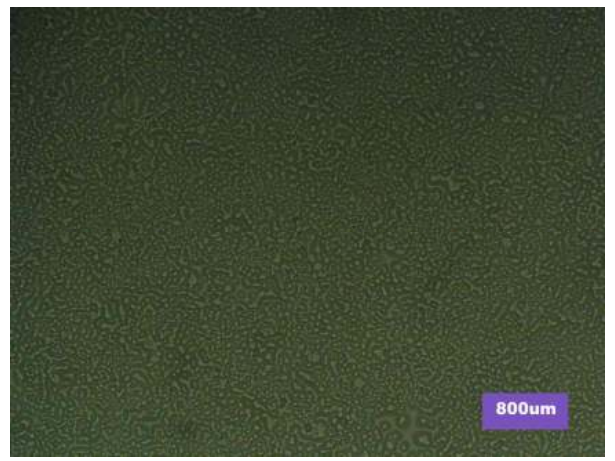

3\% Teflon:PGMEA:PPG = 3:1.5:0.75 at $10 x$

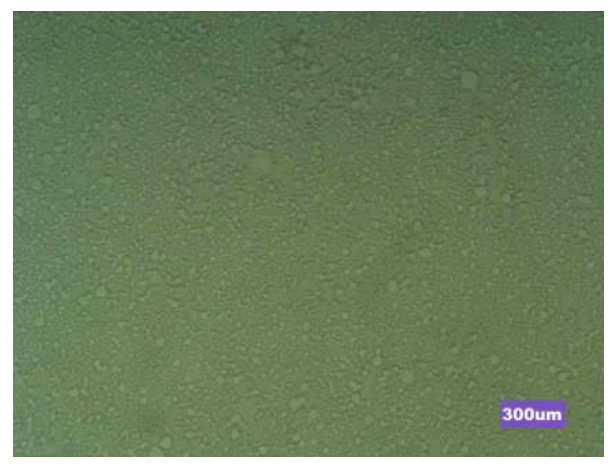

3\% Teflon:PGMEA:PPG = 3:1.5:0.75 At $20 \mathrm{X}$ (Contrast is very bad when glass samples are used)

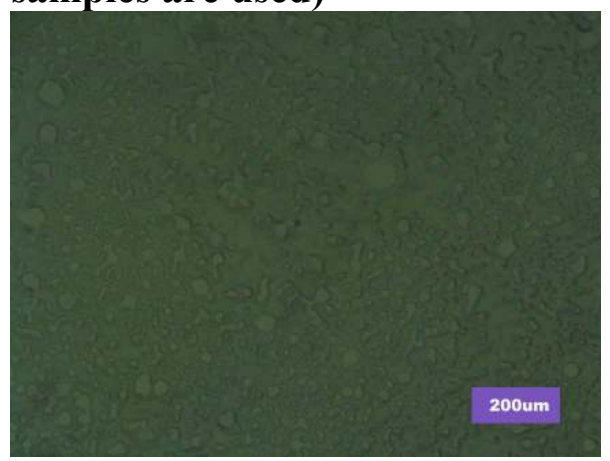

3\% Teflon:PGMEA:PPG $=3: 1.5: 0.75$ at 40x

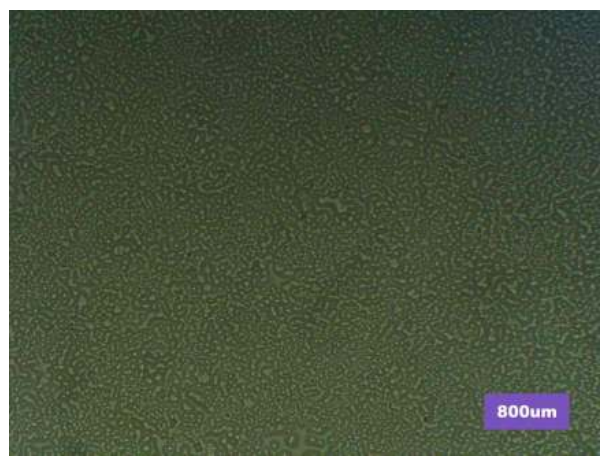

3\% Teflon:PGMEA:PPG = 3:1.5:0.75 at 10x

Table 5-14 Porous Teflon films using Teflon:PGMEA:PPG in different ratios 
5.7.4 After removing excess PGMEA from solution and only keeping the milky solution

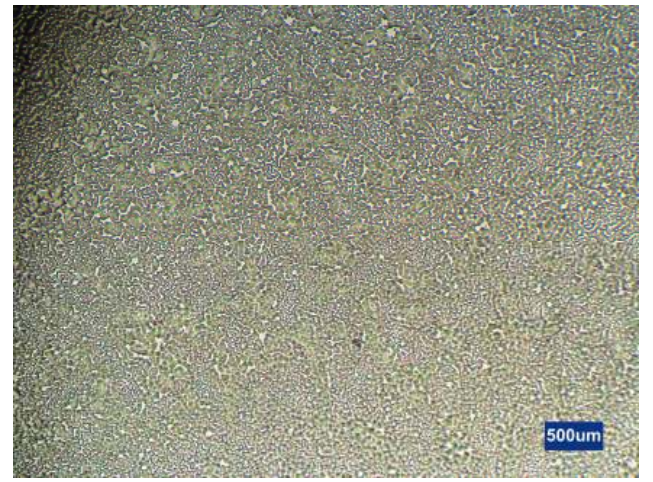

After removing excess PGMEA - At 10X

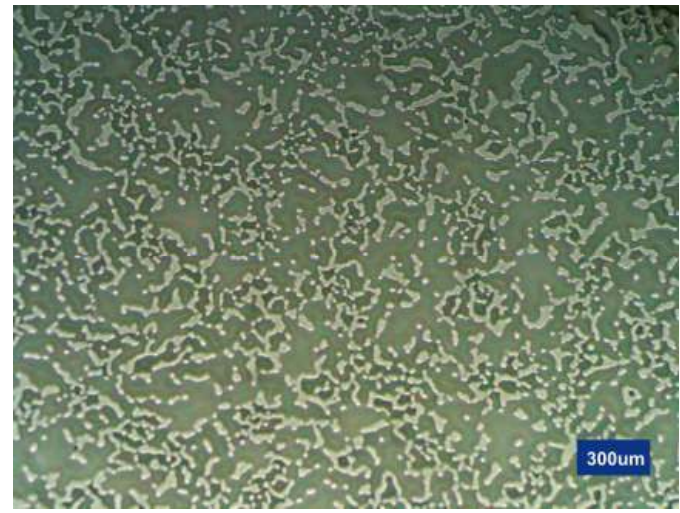

Silicon substrates - good contrast observed) At 20X

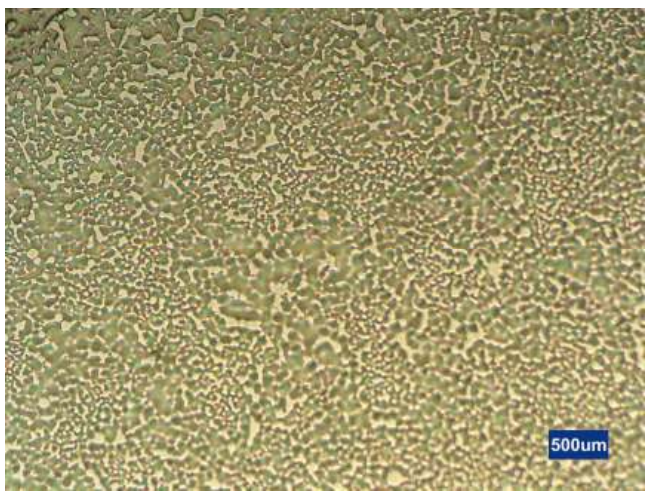

(Using Silicon substrates - good contrast observed) At $10 X$

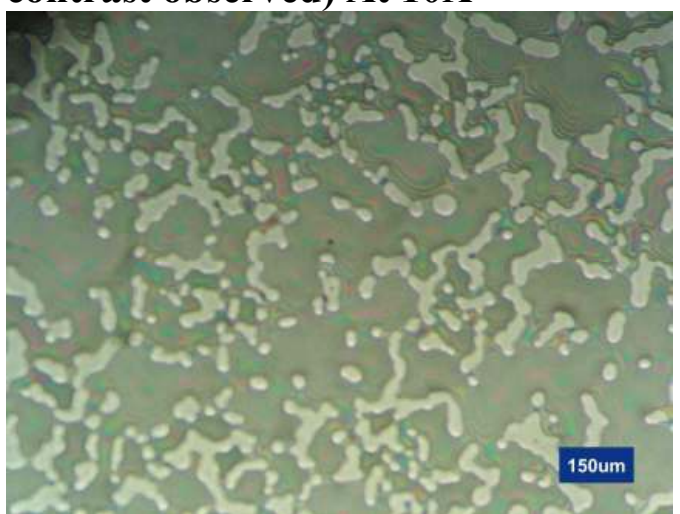

Silicon substrates - good contrast observed) At 40X

Table 5-15 Porous Teflon films after draining off the excess PGMEA

5.7.5 Contact Angles for the above substrates

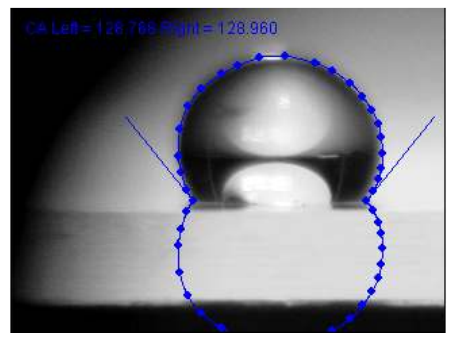

CA 129

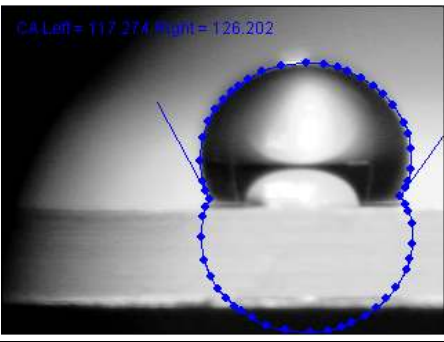

CA 117 126

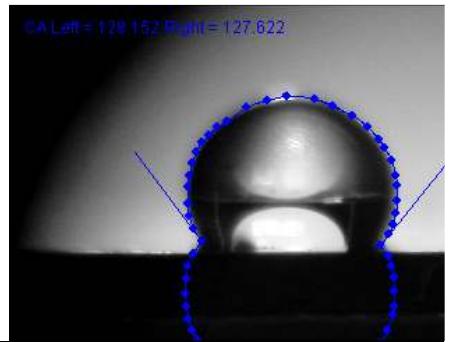

CA 128 128

Tabl

e 5-16 CA Images of Porous Teflon films after draining off the excess PGMEA 


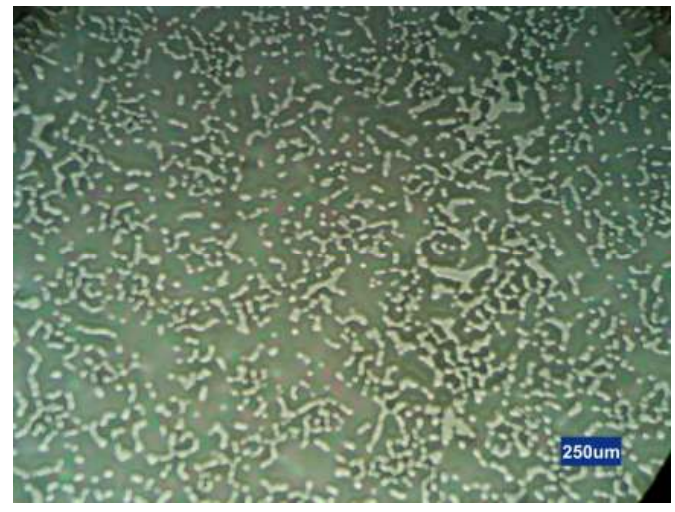

3\% Teflon:PGMEA:PPG = 3:3:3 at $20 \mathrm{X}$

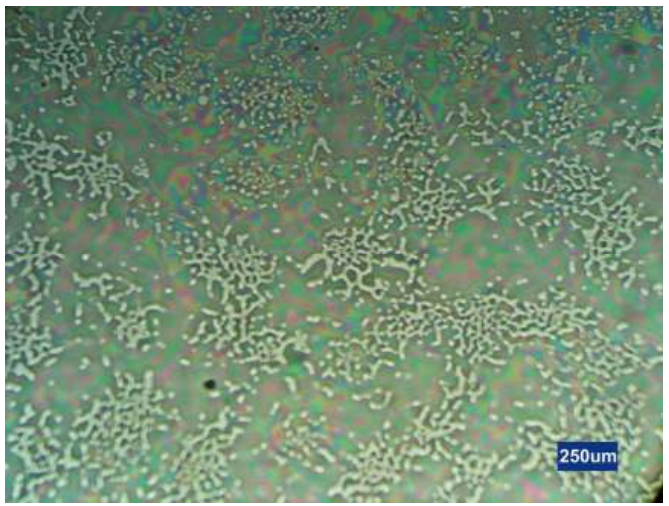

3\% Teflon:PGMEA:PPG = 3:0:3 At 20X

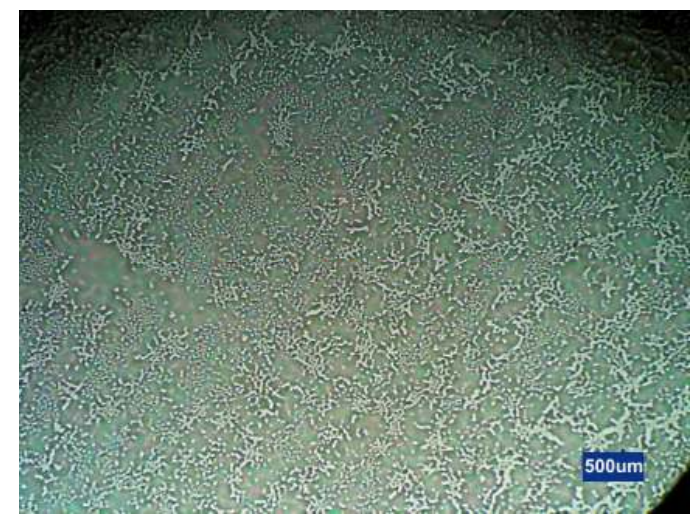

3\% Teflon:PGMEA:PPG = 3:0:3 At $10 \mathrm{X}$

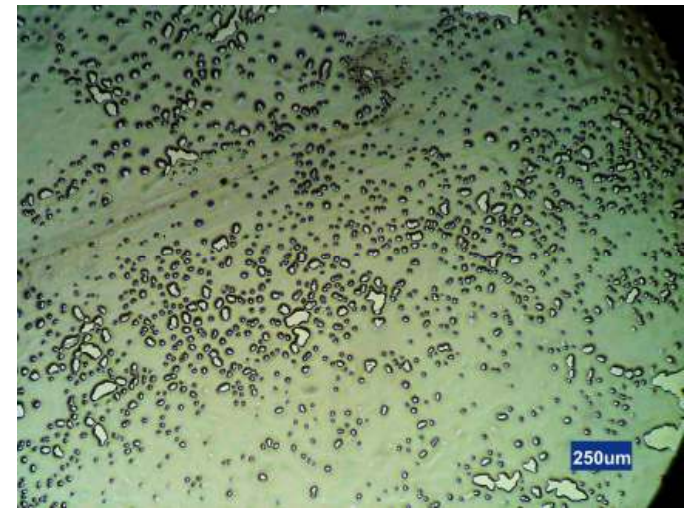

Ratios Teflon:PGMEA:PPG = 3:0:0.15 at 20X

Table 5-17 Porous Teflon films after draining off the excess PGMEA

5.7.6 CA Images of films using the Teflon:PGMEA:PPG $=3: 1.5: 3$ ratios

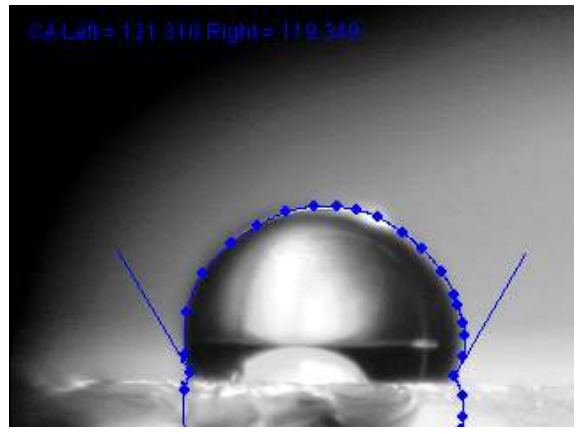

CA 122 121

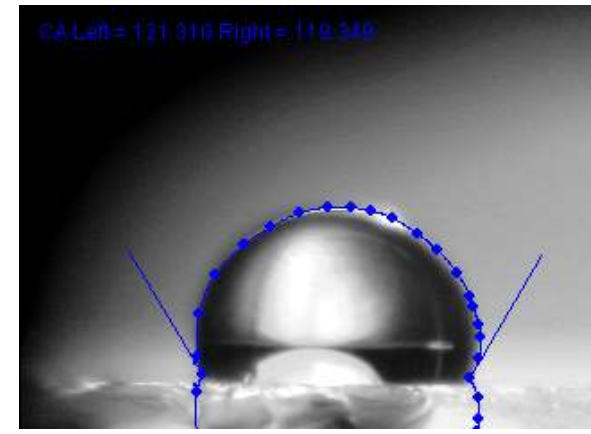

CA 121 119

Table 5-18 CA Images of Porous Teflon films after draining off the excess PGMEA 


\subsubsection{CA Images of films using the Teflon:PGMEA:PPG $=3: 3: 1.5$ ratios}

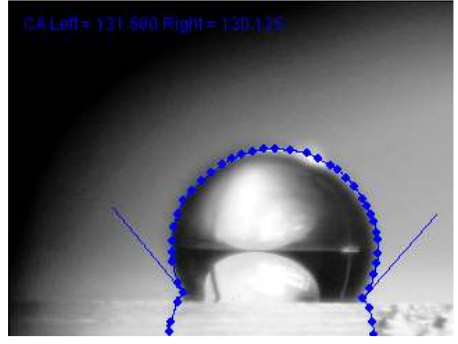

CA 132 130

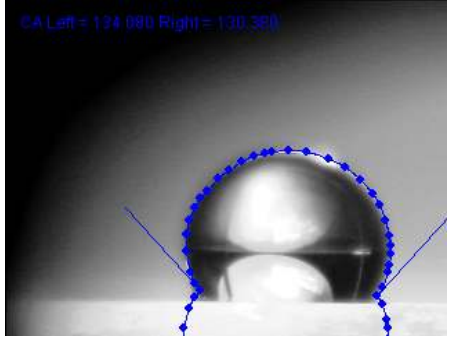

CA 134 130

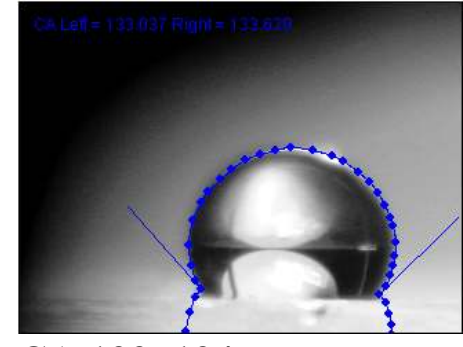

CA 133 134

Table 5-19 CA Images of Porous Teflon films after draining off the excess PGMEA

\subsubsection{Discussion on porous Teflon films using micro-emulsions}

When non-solvents such as ethanol, iso-propanol, MEK or water are added to polymeric solutions, the polymers are seen to precipitate[67] due to the increase in the polymer phase separation between the two phases of non-solvent plus the good solvent and the polymer plus small amounts of the good solvent. Two macroscopic phases are seen at the completion of the process: one polymer rich and the other polymer poor. It is seen that crystallization starts in the polymer rich phase by the formation of crystal nuclei with further development into spherulites, fibrilates and other crystal shapes[67]. Smaller spherulites result due to the increase in nucleation rate, due to the presence of nonsolvents. Also since in most cases the non-solvents are more volatile than the solvents, faster evaporation leads to shorter times for crystal formation. Also at times it is seen that non-solvents containing oxygen increase the wettability of the polymer solution on the substrate due to the presence of $-\mathrm{OH}$ groups whenever glass substrates are used resulting in an initial homogenous layer over which the network grows forming a much more homogenous final coating. 
As can be seen in the above images of porous Teflon films using the micro-emulsion process, the contact angles could not be enhanced by any significant amount as compared to smooth Teflon films. A more rigorous and extensive study would need to be done before the porosity developed could be uniform across the films and the pore sizes could be controlled. 


\subsection{Teflon AF 1600 films embedded with SiC Nano-particles}

\subsubsection{2\% Teflon Films embedded with 0.1\%(wt\%) SiC Nano-particles}

In this experiment, Teflon films were loaded with $130 \mathrm{~nm}$ sized surface modified $\mathrm{SiC}$ nano-particles embedded in $2 \%(w t \%)$ Teflon solutions. It can be shown using AFM nano-indentation that teflon films loaded with $\mathrm{SiC}$ nano-particles are stronger in terms of hardness than teflon films without these particles. Also these particles help in inducing surface roughness which increase the contact angles with water. A more detailed discussion on the roughness induced with the help of these particles has been given towards the end of this section. All the SiC nano-particles that were used in the Teflon solutions were surface modified by silanising them with FSM which is a fluoroalkylsilane as described in the experimental section. This was done to lower the surface energy of the particles. Untreated particles are seen to agglomerate within the solution leading to nonhomogenous dispersions. It is also seen that such surface modification of particles helps in enhancing the filler/matrix interfacial interaction[66] and increases the integrity of the composite. 


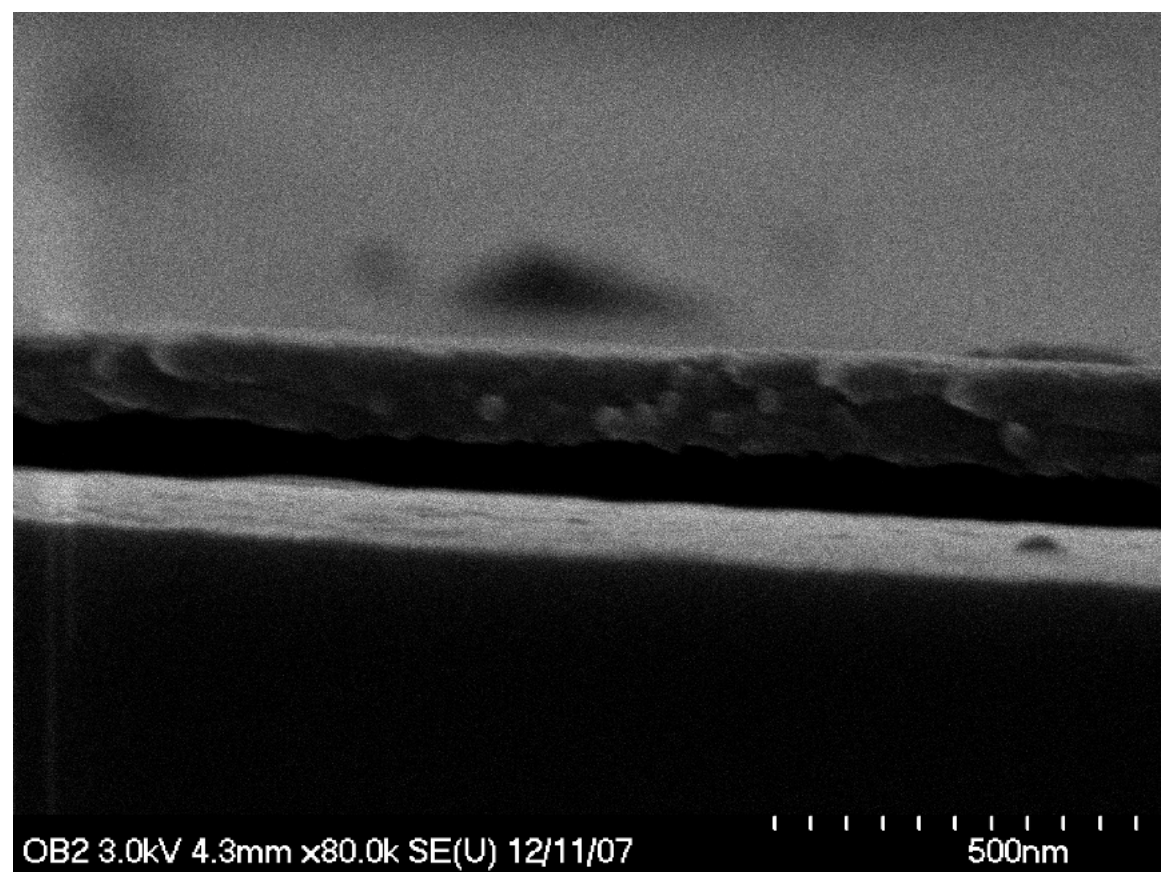

Figure 5-12 $\mathrm{SiC}$ particles can be seen embedded in the Teflon films in the images above(1)

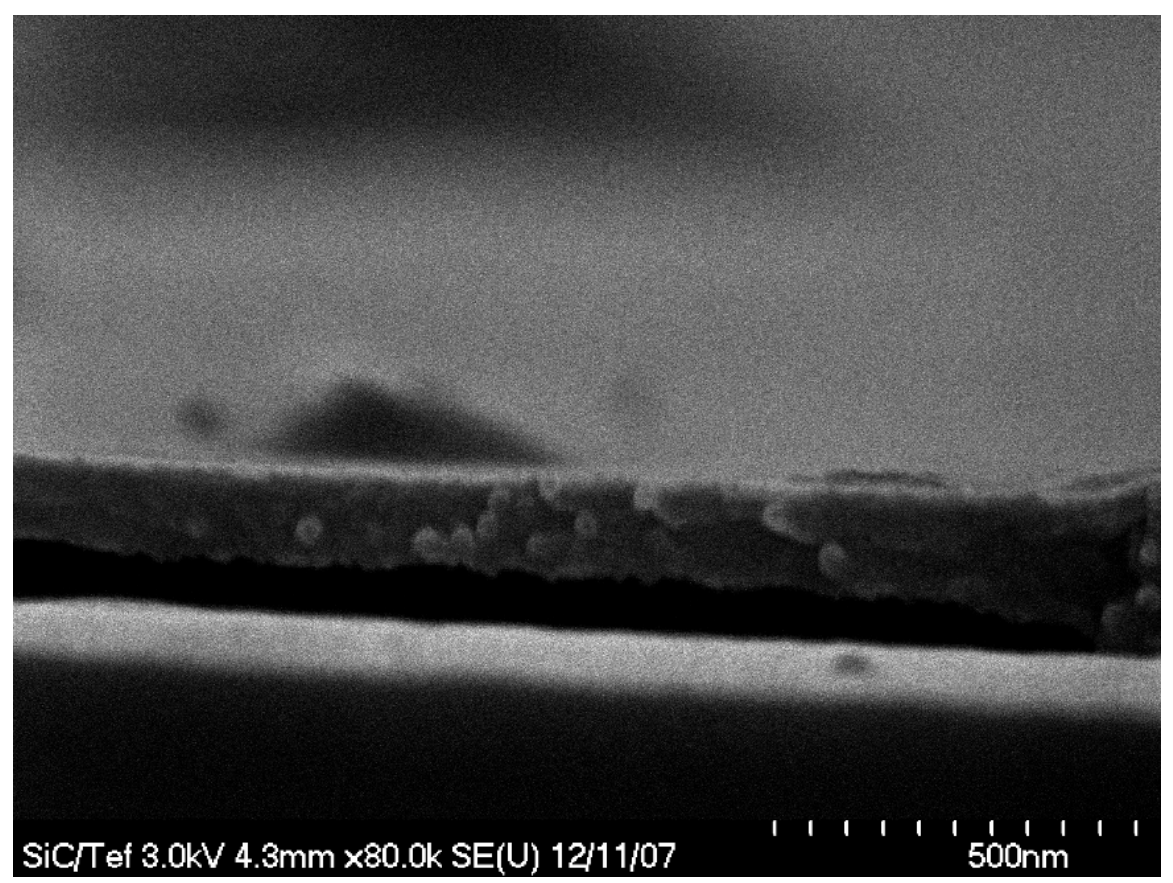

Figure 5-13 SiC particles can be seen embedded in the Teflon films in the images above(2) 


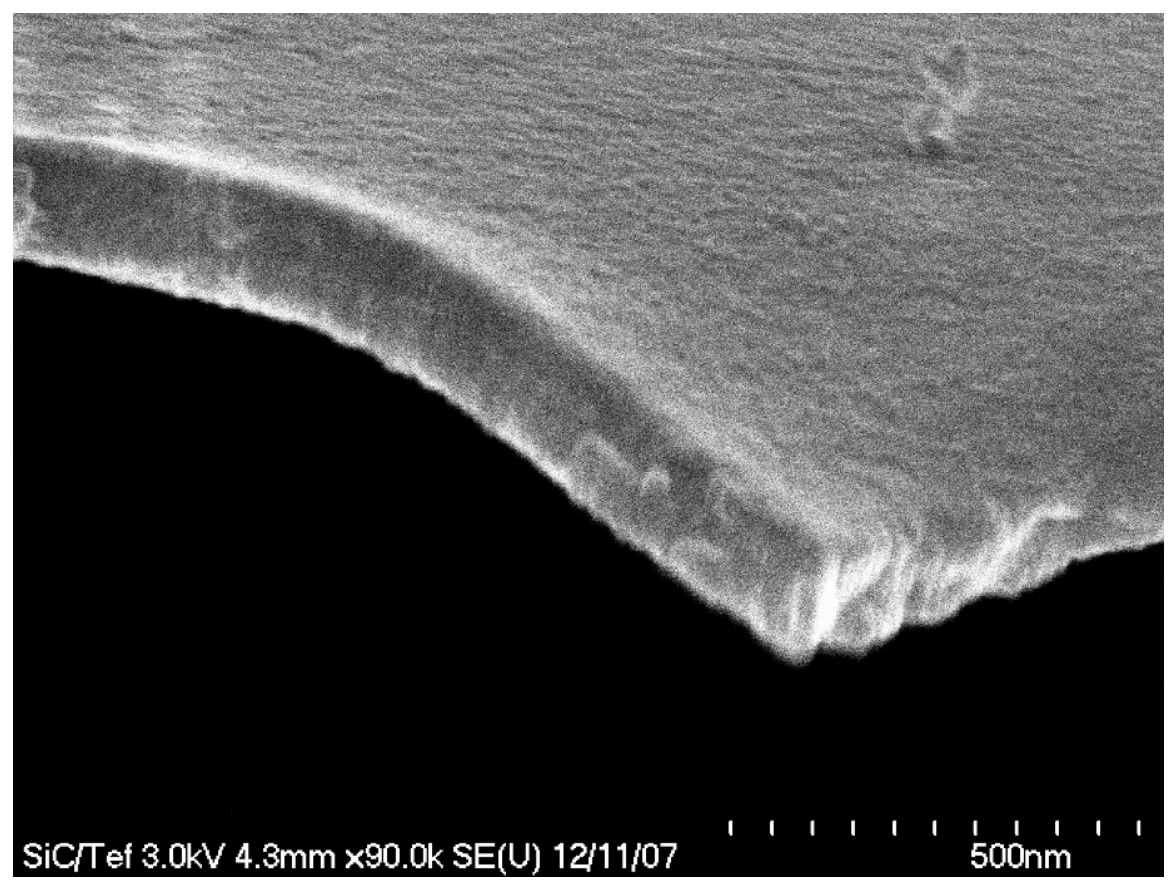

Figure 5-14 One section of the substrate where the Teflon layer has been detached from the substrate and been suspended off the substrate.

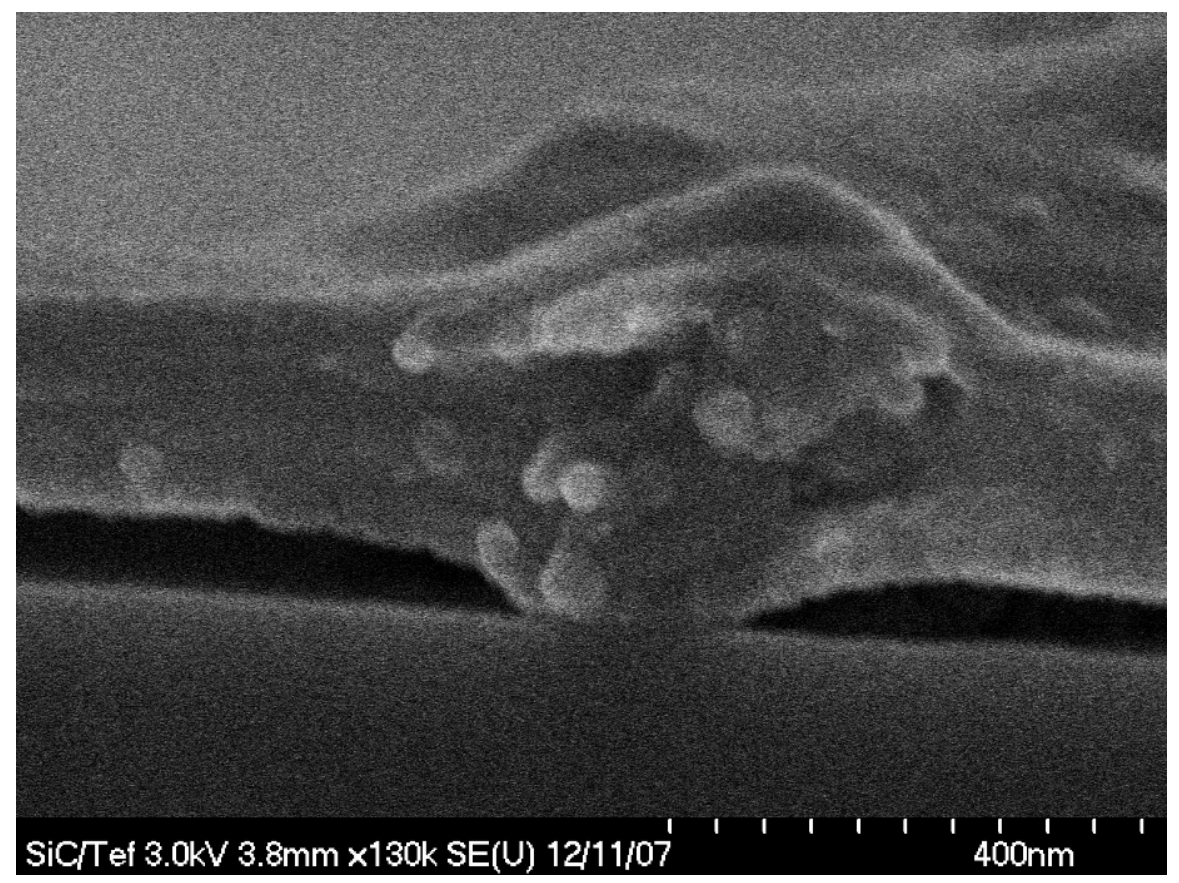

Figure 5-15 One section of the substrate where the Teflon layer did not adhere well enough to the substrate due to absence of adhesion promoter and the SiC nano-particles are seen to be clustered up. 


\section{$0.1 \%(w t \%)$ SiC embedded in $2 \%$ Teflon}

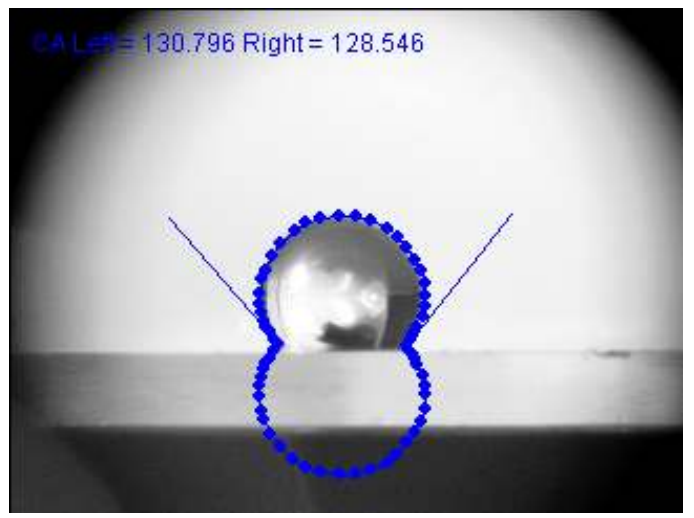

Film thicknesses in the range of 230-

$250 \mathrm{~nm}$ were obtained when the teflon

solutions were spin coated at 3500RPM.

Table 5-20 Contact angle obtained with water for the $2 \%$ Teflon films loaded with $0.1 \%(w t \%)$

$\mathrm{SiC}$

nano-particles

CA $\sim 128 \sim 130$ 


\subsection{Experiment with different wt\% $\mathrm{SiC}$ nano-particles in $0.5 \%(w t \%)$}

Teflon Solutions

In this experiment, $0.5 \%(\mathrm{wt} \%)$ Teflon Solutions were loaded with different weight percentages of $\mathrm{SiC}$ nano-particles. Spin speeds were also varied to control the thickness of films.

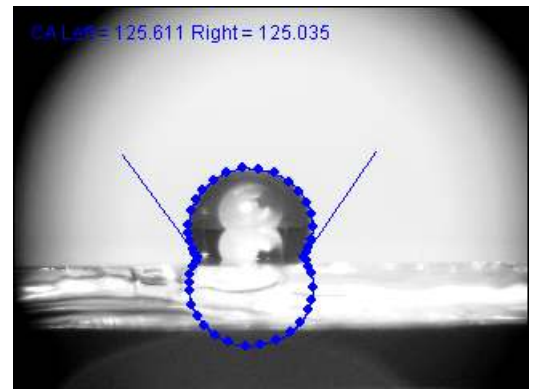

$0.1 \%(w t \%)$ SiC added, 3000RPM

\section{CA 125 126}

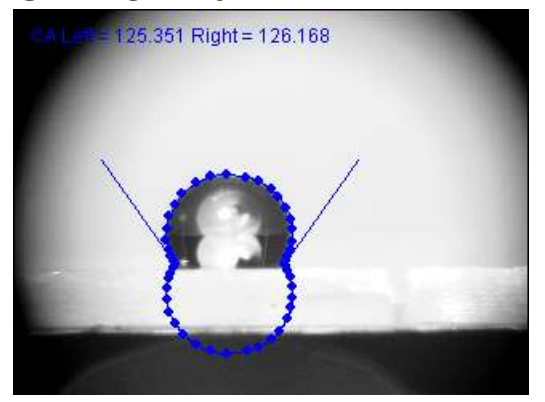

$0.1 \%(\mathrm{wt} \%) \mathrm{SiC}$ added, 4000RPM CA 125 126

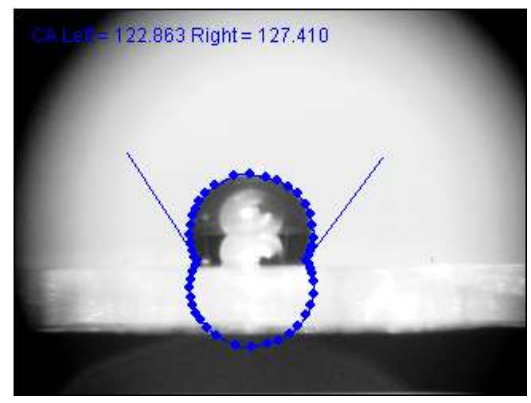

$0.25 \%(w t \%)$ SiC added, 3000RPM CA 123 127
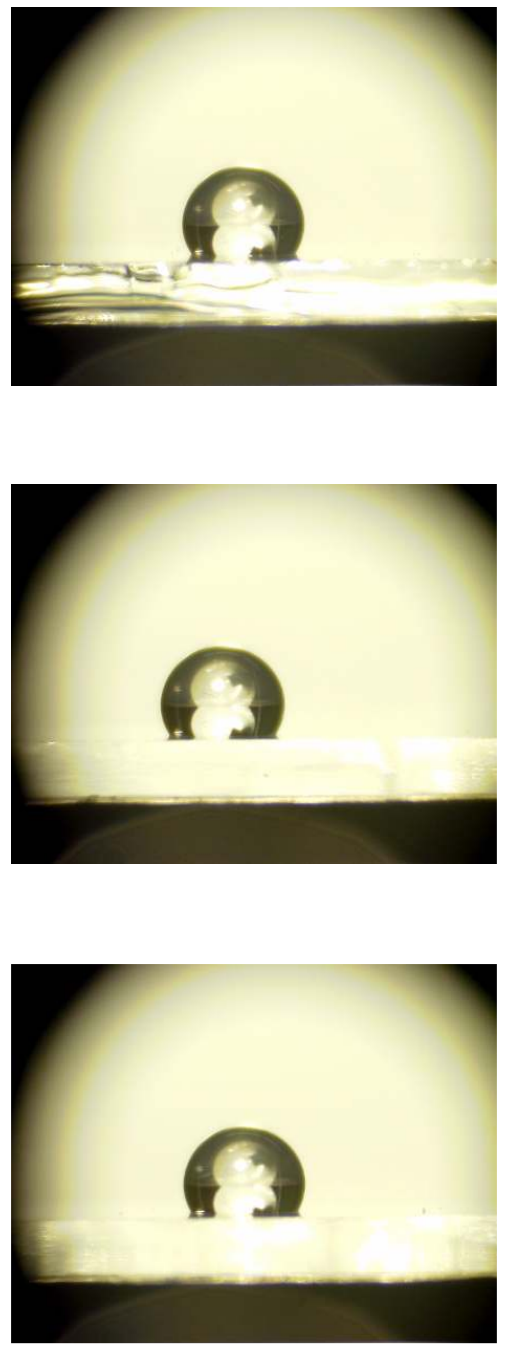

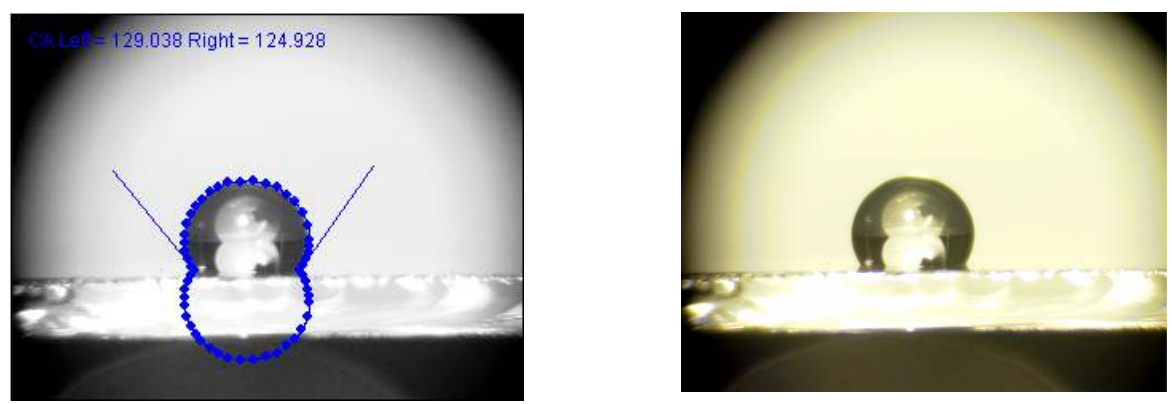

$0.25 \%(w t \%)$ SiC added, 4000RPM

CA 125 129

Table 5-21 Teflon films were embedded with different wt\% of SiC nano-particles. Spin speeds were also varied to control the thickness of films.

\subsubsection{UV-VIS transmission Spectroscopy on above films}

A UV-VIS transmission spectroscopy was carried out on the films to see the change in the transmission characteristics with different loading percentages.

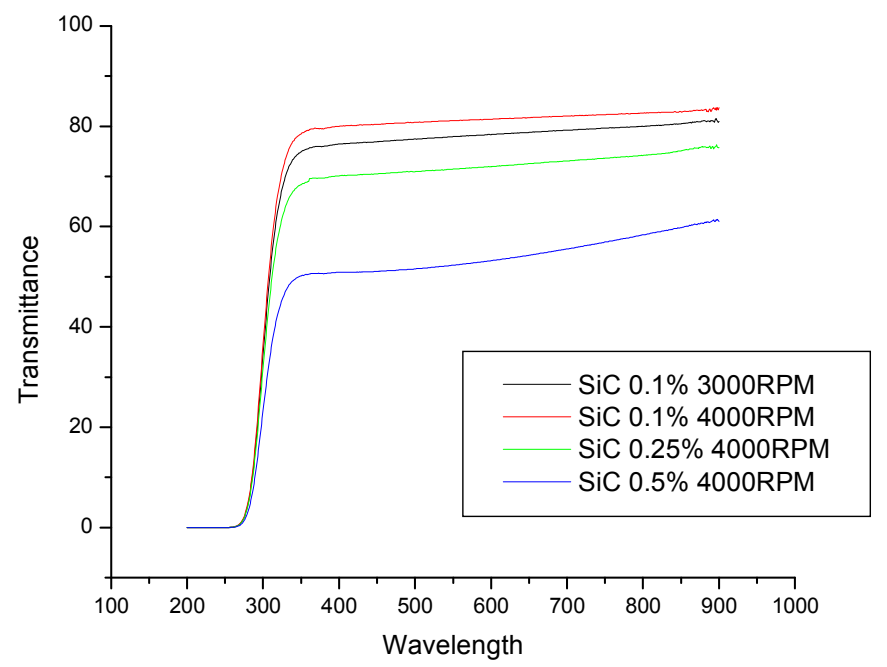

Figure 5-16 UV-VIS spectroscopy of the SiC embedded Teflon films. All the films were spinned on 'Fisher' brand glass slides.

A tape test was carried out on all the above films and all the films passed the tape test for adhesion. A 'Scotch ${ }^{\circledR}$ Magic ${ }^{\mathrm{TM}}$ tape' was used in all the above tests. As can be observed, lower percentage loadings of SiC nano-particles in the Teflon films resulted in higher 
transmittances. Also higher spinning RPM values resulted in thinner films as indicated by the above transmission characteristic graph.

\subsection{Experiment with $0.1 \%(w t \%)$ SiC nano-particles in $0.125 \%(w t \%)$ Teflon Solution}

This approach was carried out to see if the surface roughness could be enhanced with thinner coatings for the same $\mathrm{SiC}$ nano-particles content loading.

\subsubsection{Loading with $0.1 \%(w t \%)$ SiC Nano-particles}
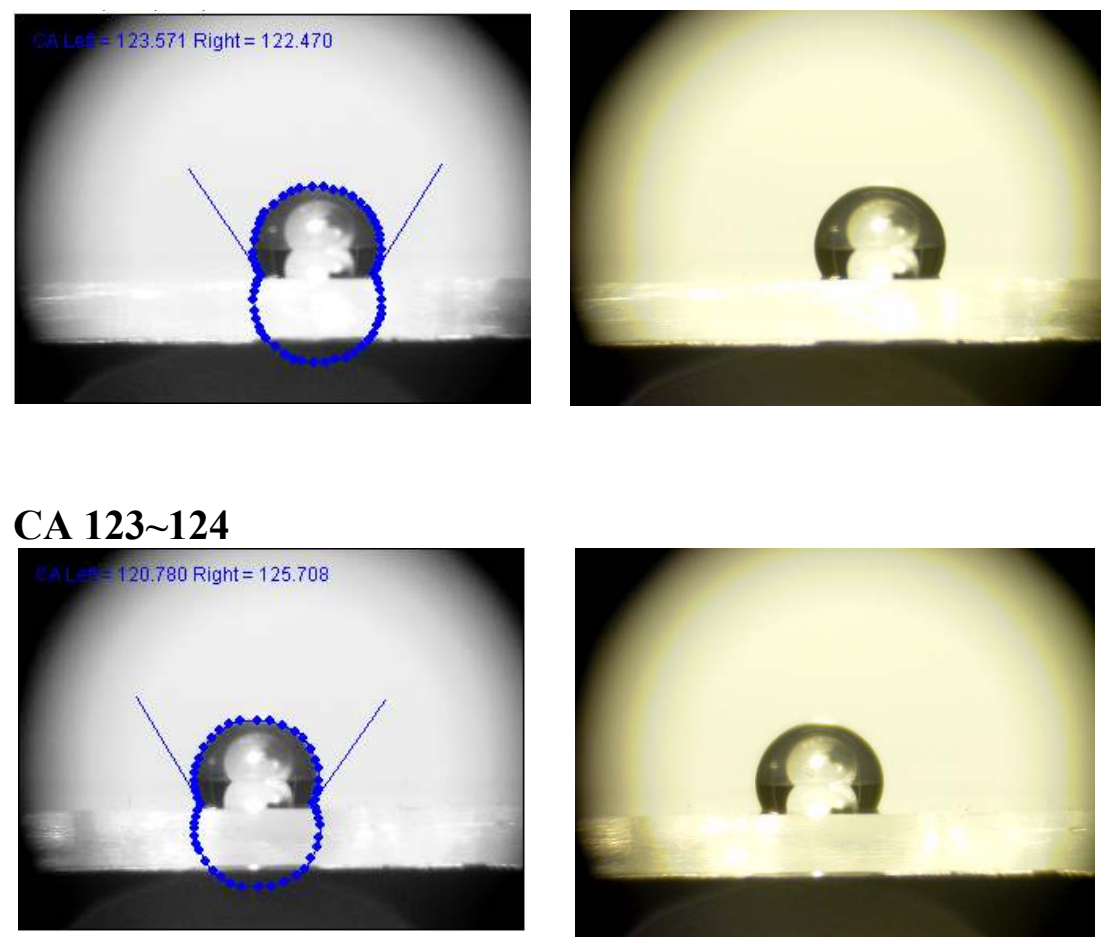

Table 5-22

CA images of $0.1 \% \mathrm{SiC}$ Nano particle embedded Teflon Films

CA 121 126

The above films were loaded with a $0.1 \%(\mathrm{wt} \%) \mathrm{SiC}$ nano-particle content. A single layer of this solution was spinned on a glass substrate. The glass substrates were FSM treated for the same reasons as mentioned above. This configuration resulted in films that were transparent but the contact angles of these films were not all that significant to assume that the droplet was residing on a composite surface rather than a 'wenzel' type surface. 


\subsection{Experiment with $0.1 \%(w t \%)$ SiC Nano-particles in $0.125 \%(w t \%)$ Teflon Solution(Triple Coat)}

In another experiment, samples were prepared wherein 3 layers were spinned consecutively on the same substrate. This was done to see if the roughness due to the $\mathrm{SiC}$ nano-particles could be enhanced without causing too much change in the thickness of the film as compared to films produced by just using a single coat.

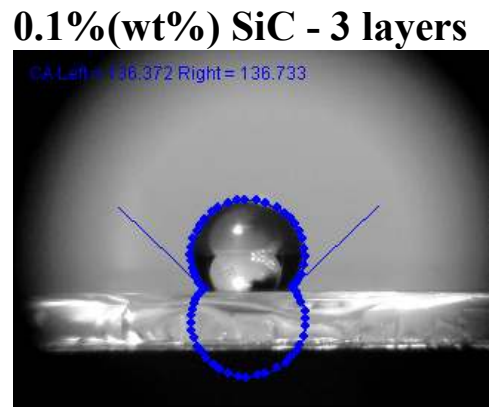

CA 123 137

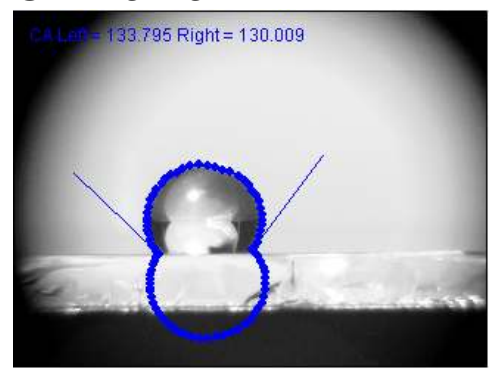

CA 134 130

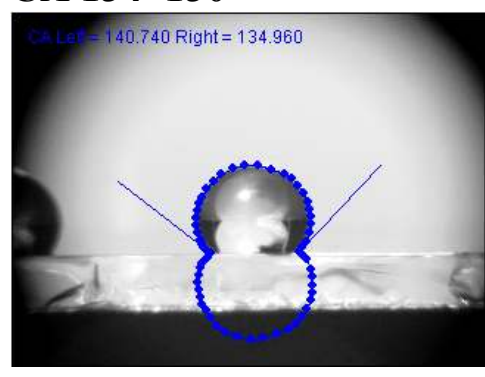

CA 135 141
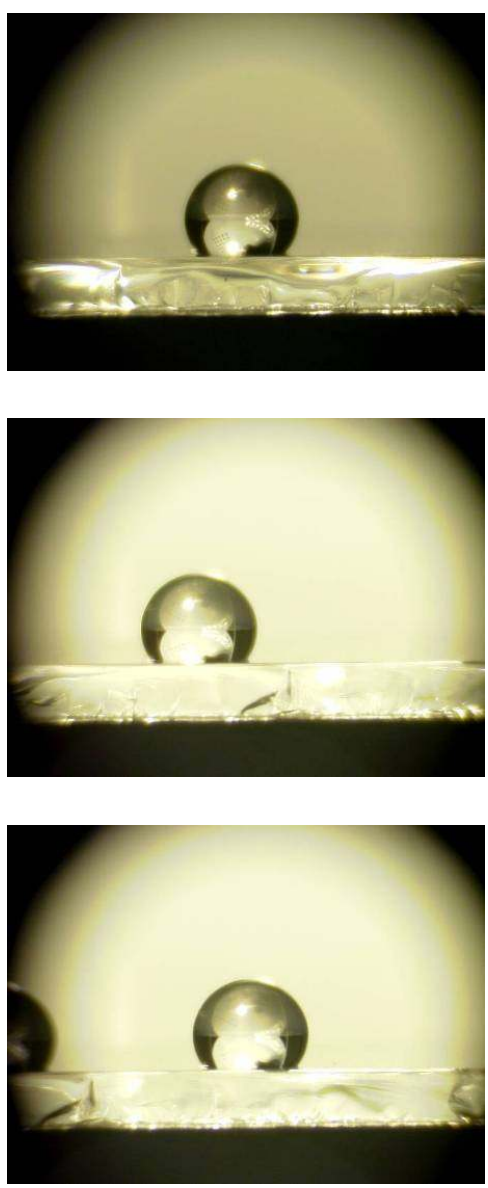

Table 5-23 CA images of $0.1 \%(w t \%)$ SiC Nano-particle embedded Teflon Films(Triple Coat)

As can be seen in the films above, the contact angles varied from 130 to 140 degrees which indeed proved to be a good thing. Spinning three consecutive layers could be 
helping by increasing the number of particles per unit area and thus increasing the surface area to a greater extent as compared to a single layer. 


\subsection{Experiment with different wt\% of $\mathrm{SiC}$ Nano-particles in $0.125 \%(w t \%)$ Teflon Solution}

Different weight percentages of $\mathrm{SiC}$ nano-particles viz. $0.25 \%(\mathrm{wt} \%)$ and $0.5 \%(\mathrm{wt} \%)$, were loaded in $0.125 \%(\mathrm{wt} \%)$ Teflon solutions to see their effects on surface roughness. In the case where $0.5 \%(\mathrm{wt} \%)$ of $\mathrm{SiC}$ nano-particles were added to the Teflon solutions, different substrates were spinned twice and thrice to see the effect of these particles on the films. The results have been given below. Each spin coat of Teflon solution is considered to be a single coat. So if a sample consists of 2 layers, it simply means that the substrate has been spin coated twice with the Teflon solution

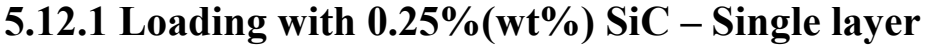
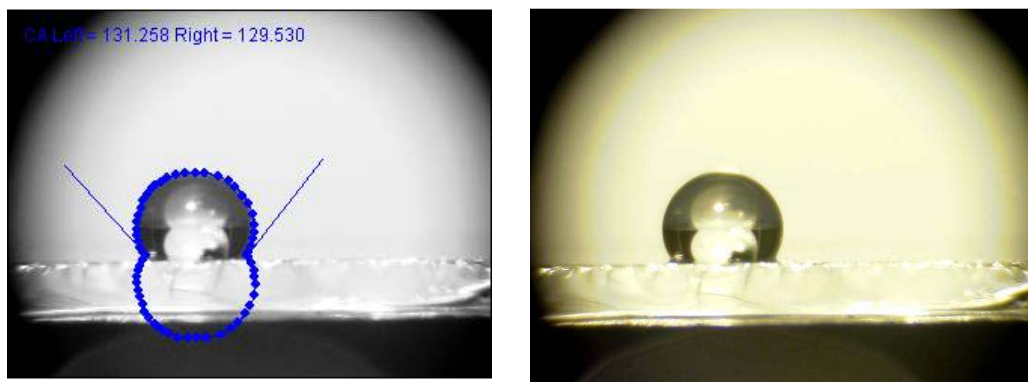

\section{CA 131 129.5}
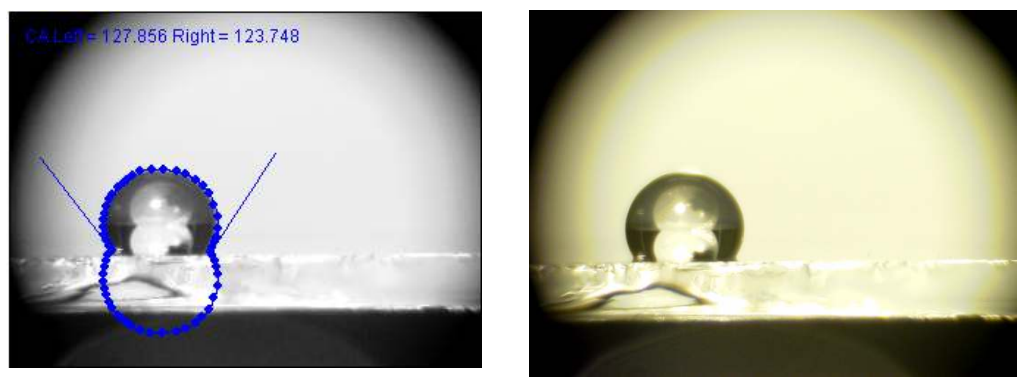

Table 5-24

CA Images of $0.25 \% \mathrm{SiC}$ Nano particles embedded Teflon Films

CA 128 124 


\subsubsection{Loading $0.5 \%(w t \%)$ SiC - Single layer}
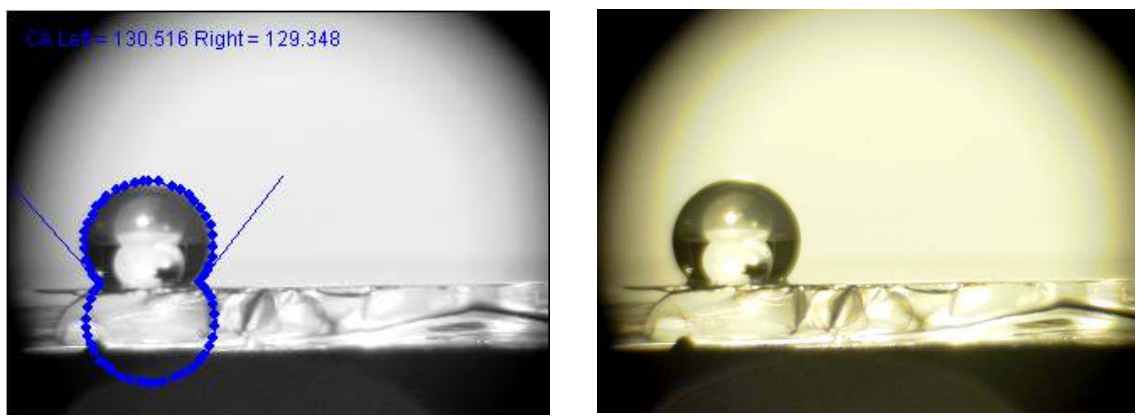

CA 129 131
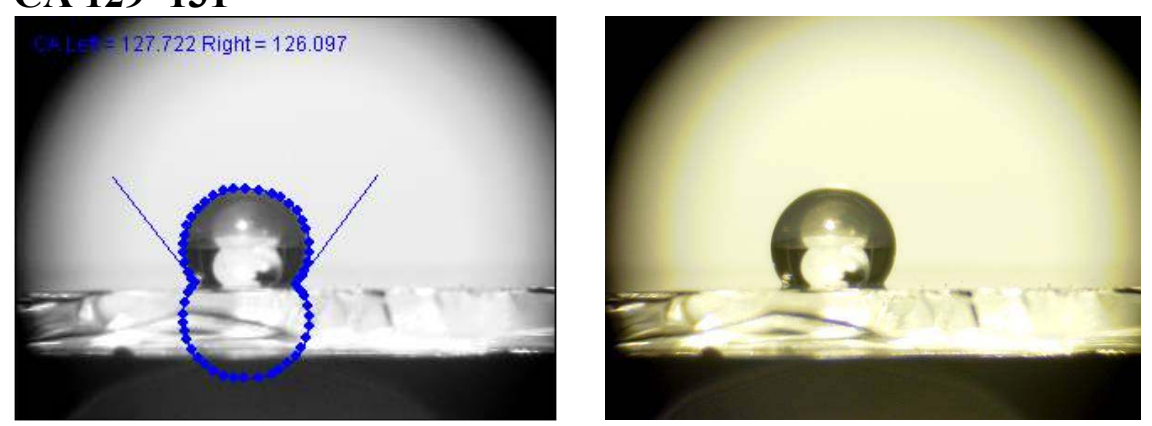

CA 126 128
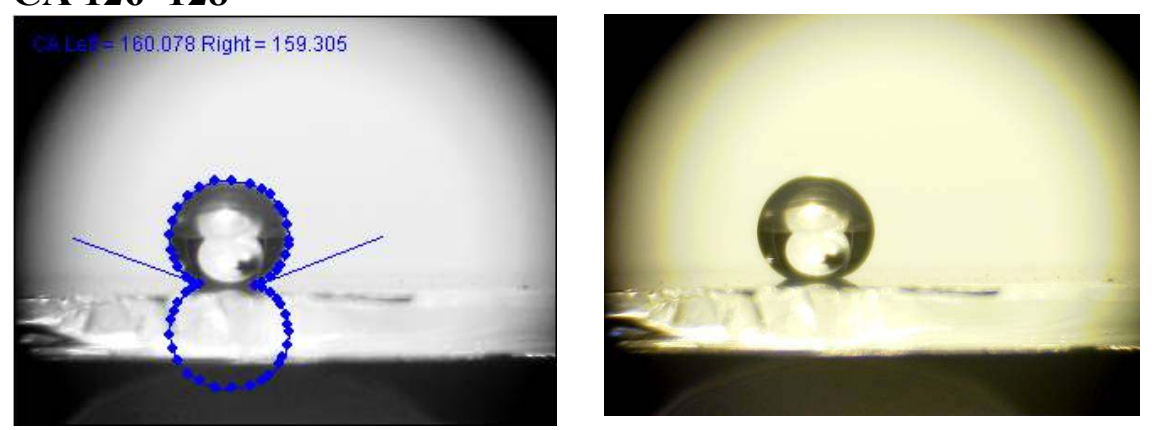

CA 159 160

Table 5-25 CA Images of 0.5\%(wt\%) SiC Nano-particle embedded Teflon Films

In this configuration i.e. $0.5 \%(\mathrm{wt} \%) \mathrm{SiC}$ is loaded in $0.125 \mathrm{wt} \%$ Teflon solution, the contact angles varied from 130degrees to 160degrees towards the edges of the substrates. It was observed that the thickness of the film is slightly thicker towards the edges of the substrates as compared to the other areas when spin coated due to the accumulation of the nano-particles at the edges. Coating the film with additional layers was decided after this observation was made as increasing the thickness in this fashion could result in superhydrophobicity. 


\subsubsection{Loading with $0.5 \%(w t \%) \mathrm{SiC}$ - Triple layered}

In this experiment a triple coating, wherein $0.5 \%(\mathrm{wt} \%) \mathrm{SiC}$ was loaded in $0.125 \%$ Teflon solution, was used to make the films. This configuration resulted in super-hydrophobic films. Some information on these films are given below.

\section{$0.5 \%(w t \%) \mathrm{SiC}-3$ layers}

[Picture could not be taken as the drop could not stabilize on the surface indicating superhydrophobicity]

In the above configuration, 3 layers were spin coated consecutively before any further processing.

\section{$0.5 \%(w t \%) \mathrm{SiC}-2$ layers}

[Picture could not be taken as the drop could not stabilize on the surface indicating superhydrophobicity]

Again, as before the same solution was spin coated twice consecutively before any further processing. 
5.12.4 Transmission Spectra of the above $[0.5 \%(w t \%)$ SiC loaded in $0.125 \%(w t \%)$ Teflon solution(dual coat)] Super-hydrophobic films

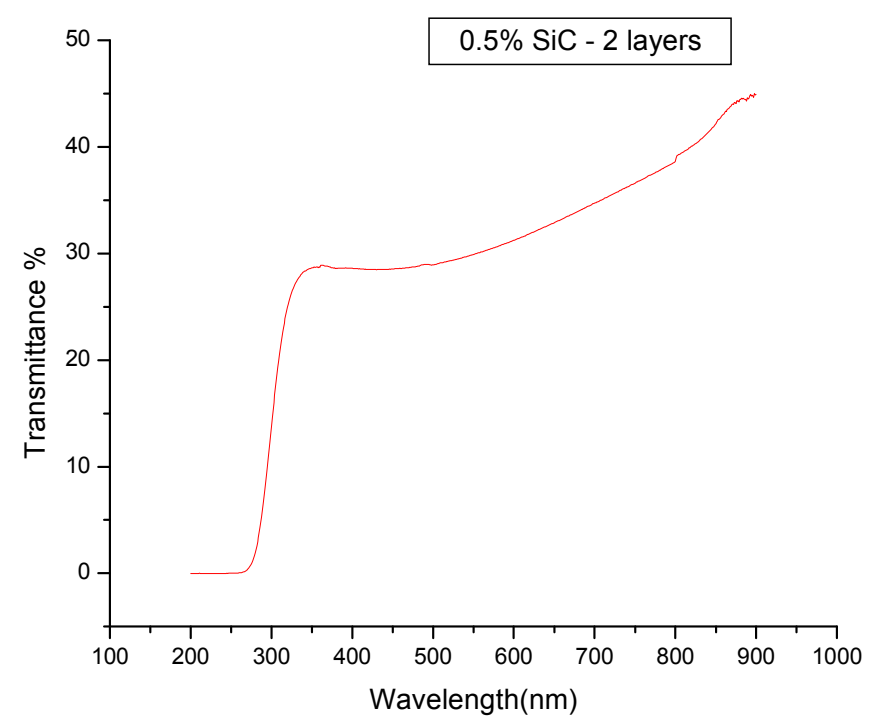

Figure 5-17 UV-VIS spectroscopy of dual coated 0.5\%films.

All the films were spinned on 'Fisher' brand glass slides.

As can be seen in the above transmission characteristics, transmission in the visible was below $40 \%$ which is not feasible in applications where transmission is important. Hence obtaining thinner coatings which would possess higher transmissions was required. 


\subsection{Experiment with $0.0625 \%(w t \%)$ Teflon Solution}

Since the transmittance fell drastically to about $30 \%$ when $0.5 \%$ by weight of $\mathrm{SiC}$ nanoparticles were used, the same experiment was repeated using a $0.0625 \%(\mathrm{wt} \%)$ of Teflon solution. This was done so that thinner films could be obtained with higher transmittances.

In the films below, different weight percentages of $\mathrm{SiC}$ were added to $0.0625 \%(\mathrm{wt} \%)$ Teflon solutions. The Teflon solutions were made the same way as before.

\subsubsection{5\%(wt\%) SiC - Single layer}
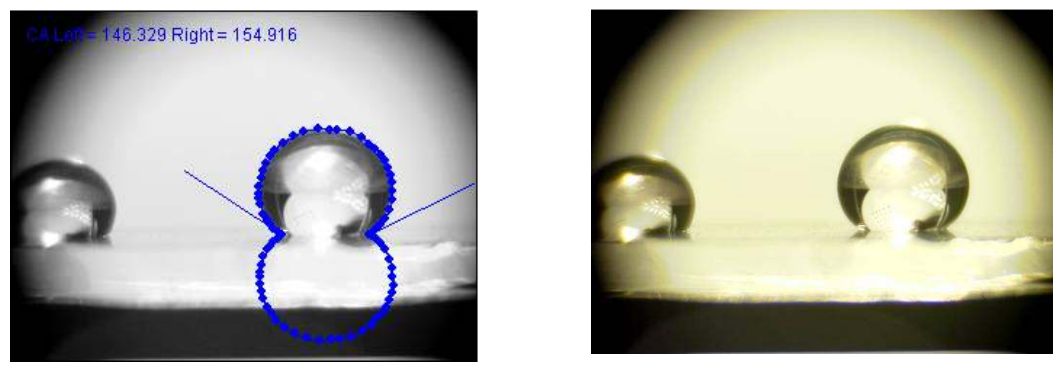

\section{CA 126 155}
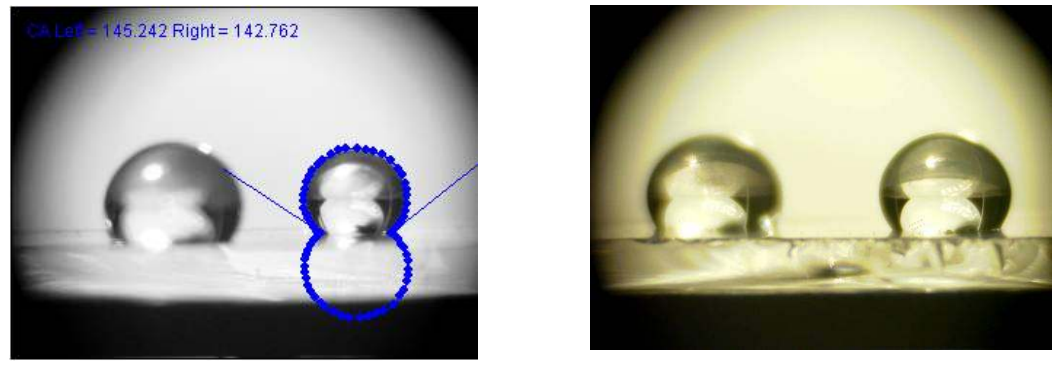

\section{CA 145 143}

Table 5-26 CA images of $0.5 \%(w t \%)$ SiC embedded in $0.0625 \%$ Teflon 


\subsubsection{Transmission Spectra of $[0.5 \%(w t \%)$ SiC embedded in $0.0625 \%(w t \%)$ Teflon solution] Super-hydrophobic films}

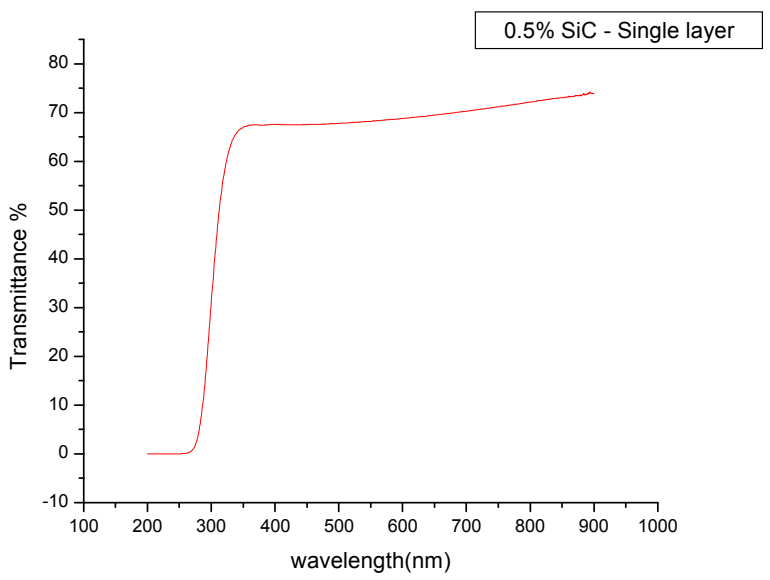

Figure 5-18 UV-VIS spectroscopy of Single Layer $0.5 \% \mathrm{SiC}$ in $0.0625 \%$ Teflon. All the films were spinned on 'Fisher' brand glass slides.

When a single layer is spinned using a $0.0625 \%(\mathrm{wt} \%)$ Teflon solution loaded with $0.5 \%(\mathrm{wt} \%) \mathrm{SiC}$ nano-particles, the films show super-hydrophobic characteristics for few seconds and then the contact angles start decreasing attaining the contact angle of the glass substrate. This could possibly be due to the seepage of water through the films which could be exposing the underlying substrate.

\subsection{Experiment with Dual Layer with different compositions}

In another experiment, a dual coat of the solution was applied to a substrate. The first layer consisted of a $0.5 \%$ by weight $\mathrm{SiC}$ nano-particles in a $0.0625 \%$ Teflon solution. The subsequent layer consisted of a $0.1 \%$ by weight $\mathrm{SiC}$ nano-particles in a $0.0625 \%$ Teflon solution. This was done to see if the coating could further be improved as far as the transmission was concerned. The dual coat exhibited super-hydrophobic characteristics. The corresponding transmission characteristics of the film have been shown below. 
$5.14 .1[0.5 \%(w t \%) \mathrm{SiC}$ in $0.0625 \%$ Teflon $]+[0.1 \%(w t \%) \mathrm{SiC}$ in $0.0625 \%$ Teflon ](double layer)

[contact angle could not be imaged as drop could not be stabilized indicating super-hydrophobicity]

On another substrate, two coats of $0.0625 \%$ Teflon containing $0.1 \%$ by weight $\mathrm{SiC}$ nanoparticles were spinned one after the other on a $0.0625 \%$ Teflon film with $0.5 \% \mathrm{SiC}$ loading.

$5.14 .2[0.5 \%(w t \%) \mathrm{SiC}$ in $0.0625 \%$ Teflon $]+[0.1 \%(w t \%) \mathrm{SiC}$ in $0.0625 \%$ Teflon $]+$ $[0.1 \%(w t \%) \mathrm{SiC}$ in $0.0625 \%$ Teflon ](triple layer)

$0.5 \%(w t \%) \mathrm{SiC}+0.1 \%(w t \%) \mathrm{SiC}+0.1 \%(w t \%) \mathrm{SiC}($ triple layer $)$

[contact angle could not be imaged as drop could not be stabilized indicating super-hydrophobicity] 


\subsubsection{Transmission Spectra of the above Super-hydrophobic films}

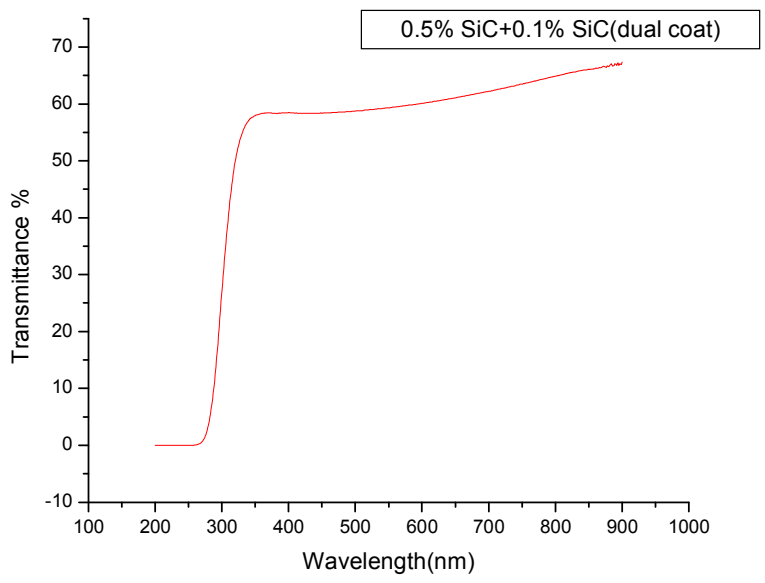

Figure 5-19 UV-VIS spectroscopy of dual Layer $0.5 \% \mathrm{SiC}+0.1 \% \mathrm{SiC}$ in $0.0625 \%$ Teflon

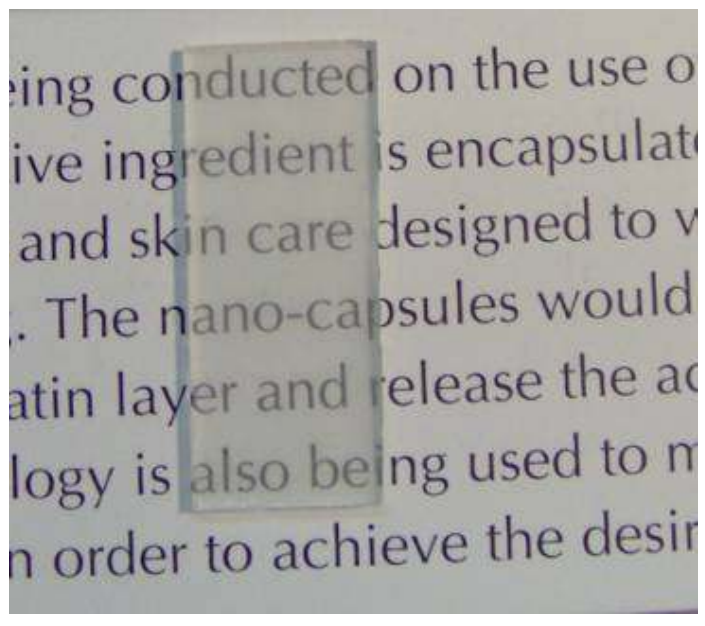

Image of the dual coated film( $[0.5 \%(w t \%) \quad \mathrm{SiC}$ in $\mathbf{0 . 0 6 2 5 \%}$ Teflon] $+[0.1 \%(w t \%) S i C$ in $0.0625 \%$ Teflon] loaded film).

These films exhibited excellent superhydrophobicity. The only disadvantage was that these films were very weak in terms of mechanical strengths and in most

cases did not survive the Scotch tape tests.

Figure 5-20 Image of the dual coated film $(0.5 \%+0.1 \%$ SiC loaded film) 
5.14.4 SEM characterization of above dual $([0.5 \%(w \mathrm{t} \%) \mathrm{SiC}$ in $0.0625 \%$ Teflon] + $[0.1 \%(w t \%) S i C$ in $0.0625 \%$ Teflon] coated film)

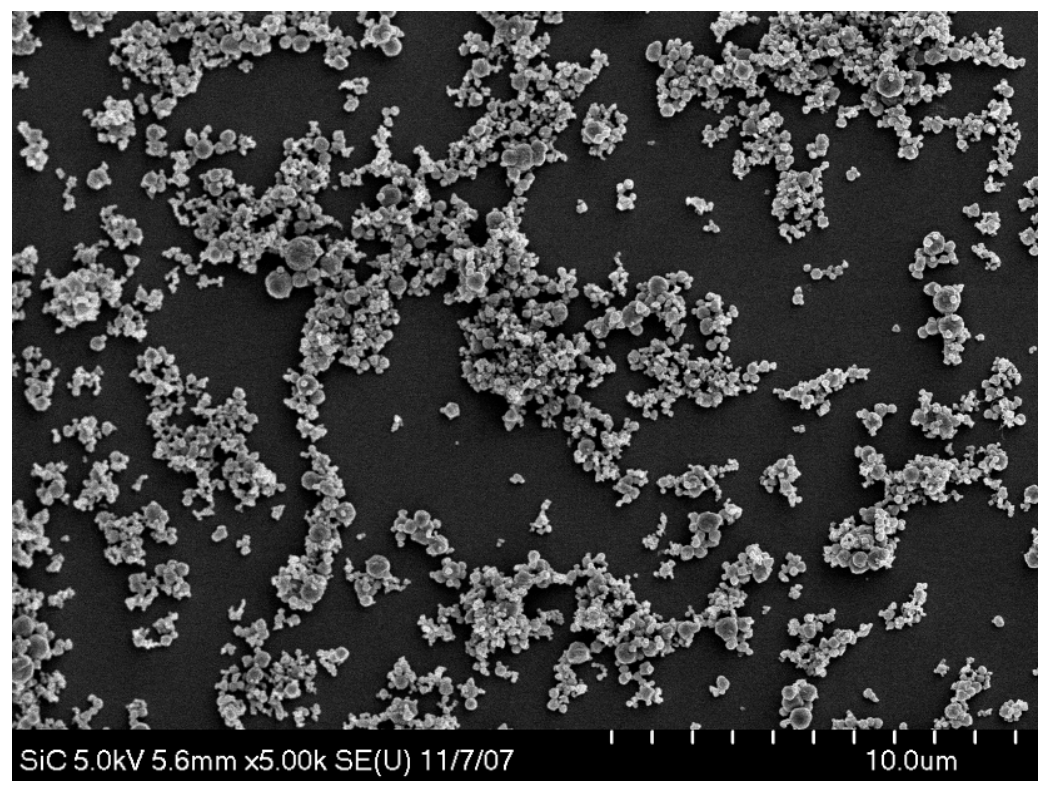

Figure 5-21 SEM images of above dual coated Superhydrophobic films(1)

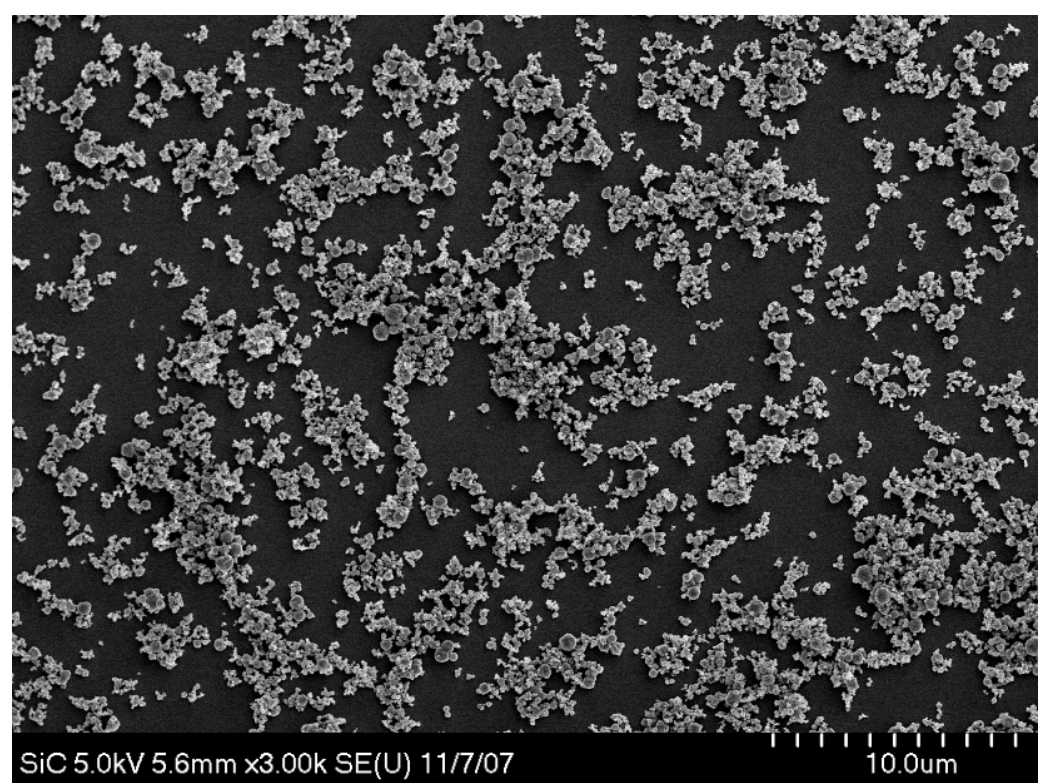

Figure 5-22 SEM images of above dual coated Superhydrophobic films(2) 


\subsection{Experiment with Silicon-Oxy Carbide Intermediate Layer}

As mentioned in the experimental section, the nano-particle embedded Teflon films were deposited on a plasma deposited Silicon Oxy Carbide layer. The cross section of this coating was observed using Scanning electron microscopy.
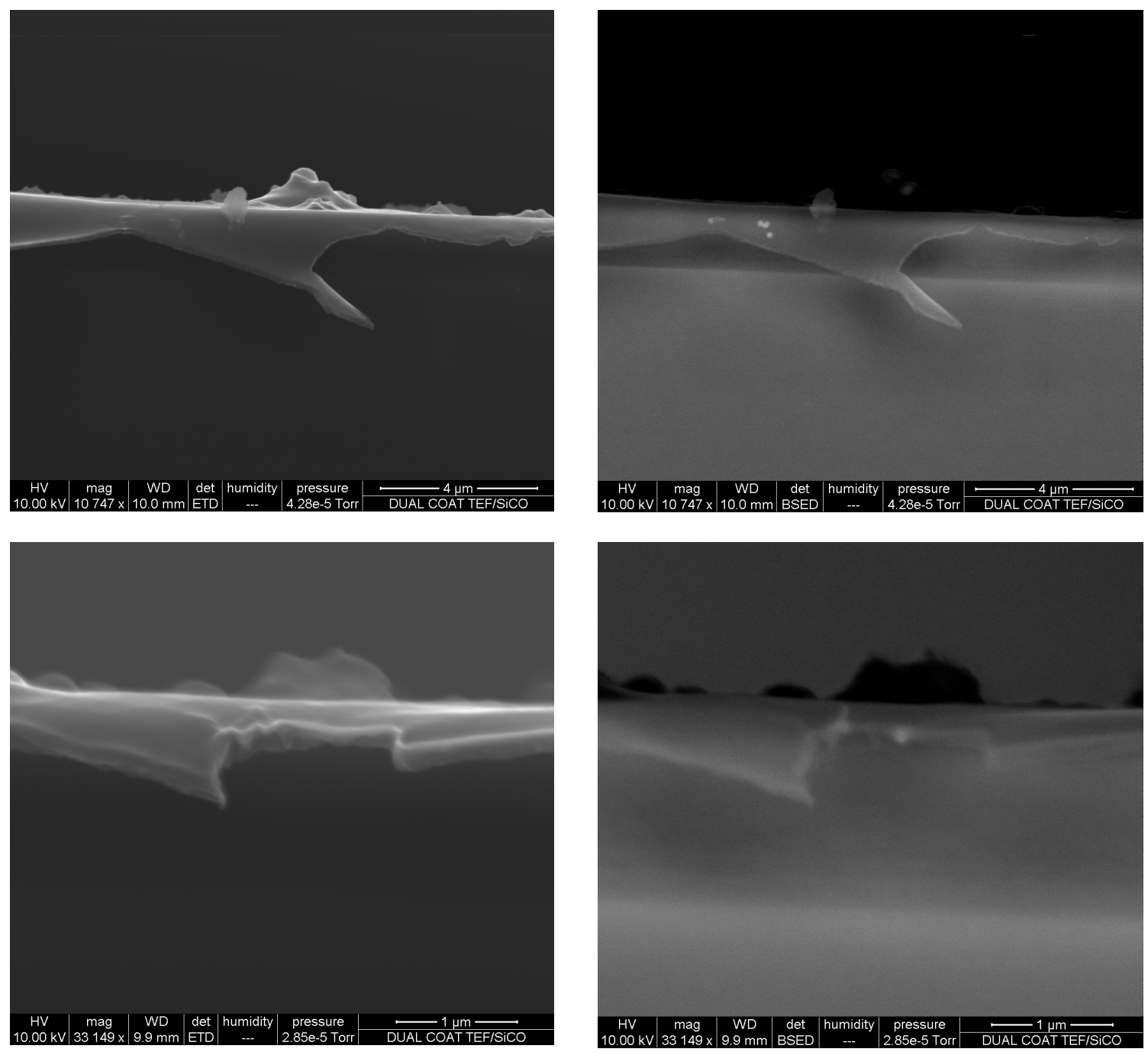

Figure 5-23 SEM and corresponding Backscattered images of SiCO/Teflon films

The difference in contrast between the glass substrate and the SiCO coating was very hard to discern. SEM Backscattered Electron Imaging was used to observe the SiCO layer. Images to the left are the normal SEM images while the ones to the right are the 
backscattered images. The thickness of this Silicon-Oxy-Carbide layer is about 650$1000 \mathrm{~nm}$. 


\subsection{Hardness Measurements}

Hardness measurements using AFM nano-indentation were also carried out on i) Teflon films w/o SiC nano-particles coated on FSM coated glass substrates ii) Teflon films w/o SiC nano-particles on Si-C-O(Silicon-Oxy-Carbide) coated glass substrates iii) Teflon films with SiC nano-particles coated on FSM coated glass substrates iv) Teflon films with $\mathrm{SiC}$ nano-particles on $\mathrm{Si}-\mathrm{C}-\mathrm{O}(\mathrm{Silicon}-\mathrm{Oxy}-\mathrm{Carbide})$ coated glass substrates. For the Teflon films, $0.1 \%(\mathrm{wt} \%) \mathrm{SiC}$ was embedded in $2 \%(\mathrm{wt} \%)$ Teflon solutions.

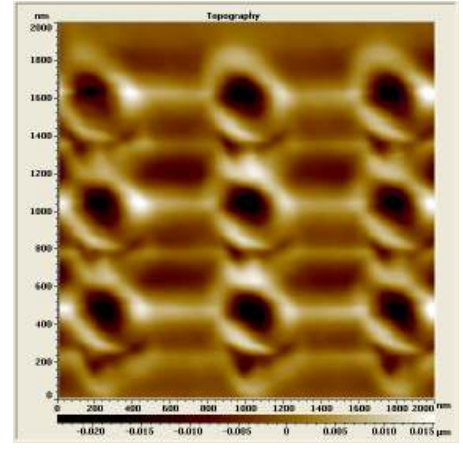

Teflon on FSM coated glass Hardness: $\mathbf{2 . 0 1} \pm \mathbf{0 . 3 M P a}$

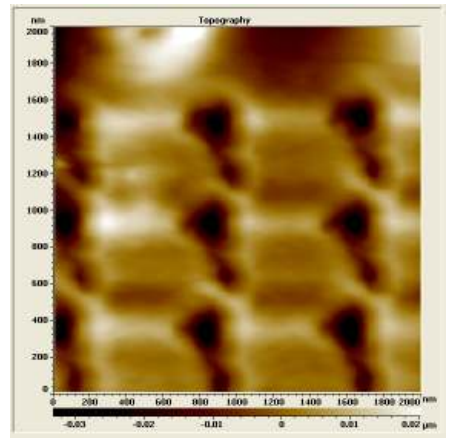

Teflon + SiC NP's on FSM coated glass - Hardness:

\section{$6.27 \pm 1.1 \mathrm{MPa}$}

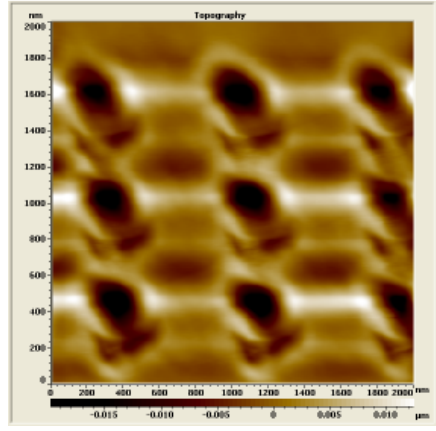

Teflon on SiCO coating Hardness: $2.14 \pm 0.26 \mathrm{MPa}$

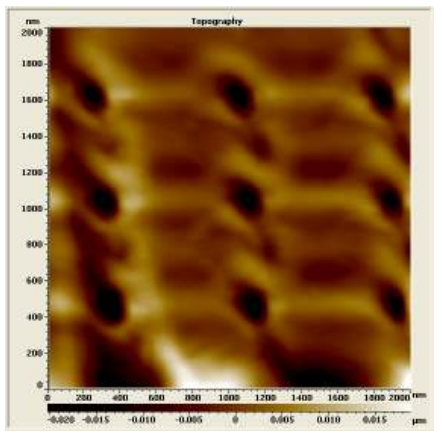

Teflon + SiC NP's on SiCO coating - Hardness:

\section{$8.46 \pm 0.83 \mathrm{MPa}$}

Table 5-27 AFM hardness measurement on SiC nano-particle embedded Teflon films. 


\subsubsection{Discussion on nano-particle loaded Teflon films}

Nano-particle loading of polymeric films have shown to increase mechanical strengths such as Young's Modulus(YM), Tensile Strength(TS) at break and dynamic hardness[63]. One study by Nakaya and Hayashi have shown that PHE(poly(bisphenol-A and epichlorohydrin)) films when embedded with modified $\mathrm{TiO}_{2}$ nano-particles were shown to have increased YM, TS at break and dynamic hardness as compared to pure PHE films. Luo, Rong and Zhang[64] investigated the incorporation of SiC nanoparticles in Polyetheretherketone(PEEK) and found that either under dry or water lubrication, wear rate and frictional coefficient were evidently lower than those of unfilled composites. Another study by C.-W. Chou et al. showed that the mechanical properties of Polyurethane-Ag composites also increased significantly as compared to virgin PU films. The loading of Teflon films with $\mathrm{SiC}$ nano-particles increases the hardness as indicated by nano-indentation from $2.14 \pm 0.26 \mathrm{MPa}$ to $8.46 \pm 0.83 \mathrm{MPa}$. besides inducing surface roughness at this percentage of loading.

\subsection{Discussion on $2 \%(w t \%)$ Teflon films loaded with $0.1 \%(w t \%)$ SiC}

Pieter van der Wal and Ullrich Steiner created super-hydrophobic surfaces[60] by using sacrificial colloids in Teflon suspensions which create arrays of depressions after annealing resulting in very rough surfaces. In order to compare their results with and without these sacrificial colloids, they prepared teflon surfaces in the absence of any sacrificial colloids. Their advancing water contact angles on these surfaces were $125 \pm 2^{\circ}$. This was few degrees in excess of smooth Teflon films having water contact angles of 110-118 degrees reported in literature which they attribute to the intrinsic roughness due 
to the Teflon grains. These results are comparable to the results which give us water contact angles of $128^{\circ}$ when $0.1 \%(\mathrm{wt} \%) \mathrm{SiC}$ particles are added to $2 \%$ Teflon solutions and deposited on SiCO coated or FSM coated glass substrates. It can be shown that the water droplet is in the Wenzel state on such surfaces with increased surface areas as compared to flat surfaces resulting in an enhanced value of the contact angle.

In the case of the $0.5 \% \mathrm{SiC} / 0.125 \%$ Teflon configuration, it was very hard to obtain the contact angles using the Contact angle measurement system. When a drop size volume between 2-5ul was used, the drop was not able to detach itself from the dispensing syringe tip. When higher volumes such as $10 \mathrm{ul}$ were used, the drop used to detach itself from the syringe tip because of the effect of gravity and just begin to roll off the substrate in spite of the measurement plane being entirely horizontal indicating sliding angles less that $1-2^{\circ}$. In the event when the droplet was made to rest on the substrate surface, while still being attached to the syringe tip, and the droplet volume was being gradually increased from 5 to $10 \mathrm{ul}$, the drop could be made to detach from the syringe tip and rest on the substrate surface resulting in a water contact angle of $156^{\circ}$. This contact angle value could be of one of the equilibrium states of lower energy when the droplet makes a transition from the Cassie regime to the Wenzel regime. Increase in the droplet energy while increasing the droplet size could make the droplet overcome the energy barrier[59] that exits between the two states. Increase in the droplet volume from 5 to $10 \mathrm{ul}$ while resting on the surface could result in the impalement of the drop by the rough textured surface resulting in a transition from a higher energy to a lower energy state. Bico et al[39] report a similar transition when the contact angle changes from $170^{\circ}$ to $130^{\circ}$ when the drop is pressed. 
Bico et al create microstructures with different morphologies on bare silicon and show that the apparent contact angles increase with decreasing area fractions, ' $\Phi$ '. They adopt a modified version of Cassie-Baxter model in obtaining their apparent contact angles for all $\theta>\pi / 2$ leaving behind the Wenzel model for hydrophilic surfaces. In creating superhydrophobic surfaces, Rob J. Klein[27] et al[62] do similar work by inducing artificial roughness with the help of fluorinated silica nano-particles on bare alumina substrates. They too show the dependence of increasing contact angles with decreasing area fractions. 


\subsection{Calculation of Solid-Air Area fractions, $\Phi$}

In an attempt to study the hydrophobicity induced by nano-particles on a rough surface, surfaces with different area fractions ' $\Phi$ ' of particles were created and characterized using contact angle measurements. The area factions were determined by analyzing the SEM images and extracting the area factions ' $\Phi$ ' using image processing.

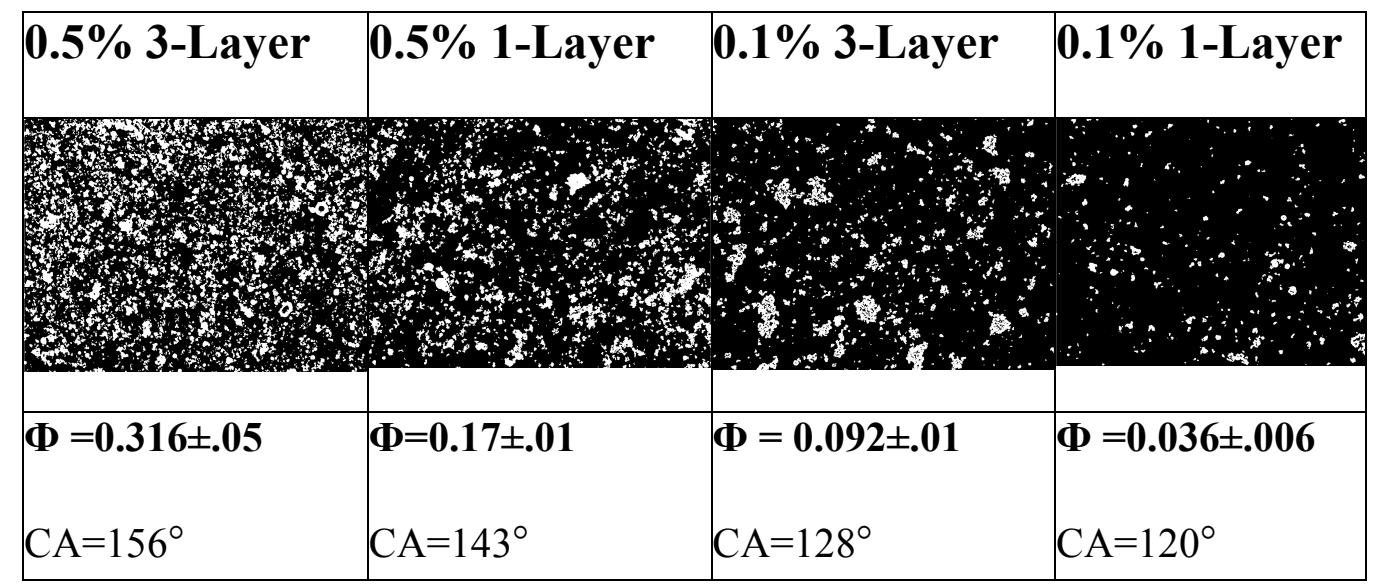

Table 5-28 Image processed SEM's with different Area fractions ' $\Phi$ '

\subsubsection{Discussion on calculation of Solid-Air area fraction}

J. Klein et al[62] calculate the area fractions of their nano-particle aided superhydrophobic surfaces by counting the number of silica spheres on a unit area from their SEM images and multiplying this number by the cross-sectional area of an average silica sphere(silica spheres observed in deep crevices were omitted from their analysis). In our work we have calculated the area fractions by doing some image processing on the SEM images. Our images were processed with an initial $1-1.5$ Pix Gaussian Blur. The image was then adjusted in such a way so as to improve the contrast between the substrate and the particles before converting them to 2 bit images. This was done so that all the pixels covering the particles could have a single value while all the pixels covering the 
substrates could have another value(either 0 's or 1's). The image was then opened in Matlab using the image processing toolbox and the 1's and 0 's were calculated. The main drawback in this approach is that the values of ' $\Phi$ ' calculated using this approach could not be the true values of the solid fractions in contact with the liquid especially when the particles are stacked on top of other particles.

\subsubsection{Apparent Contact Angles}

A comparison between the measured and the calculated apparent contact angles was made. According to Cassie-Baxter equation, $\cos \theta_{C B}=-1+\Phi_{1}(\cos \theta+1)$, the contact angles are supposed to increase with decreasing area fractions. This trend has been observed by several authors including Bico et al[39] and J. Klein et al[27]. In our measurements of apparent contact angles using the sessile drop method, we have observed that the contact angles surprisingly seemed to decrease with decreasing area fractions. As in the case of the sample designated ' $0.5 \% 3$-Layer', the measured contact angle was $156^{\circ}$ while the calculated apparent contact angle using the Cassie-Baxter equation was $147^{\circ}$. The error in this case was $11^{\circ}$. When the modified version of the Cassie-Baxter equation by Bico et al[39] which is given by $\cos \theta_{C B}=-1+\Phi_{1}(\cos \theta+1)^{2}$ was used, the apparent contact angle turned out to be $157^{\circ}$, a good agreement with the observed value.

It was impossible to get this droplet to transition to a lower energy state by pressing it which indicated that the super-hydrophobic surface that we created was very good. When pushing the drop into the structures keeping the needle close to the substrate(i.e. exerting a NON-negligible extra pressure on the drop-substrate system), it could be possible that 
the drop undergoes a transition from the Cassie state to a composite meta-stable state with partial filling of the asperities. This transition was confirmed by the increased sliding angles of more than $30^{\circ}$ as compared to previous values of less than $2^{\circ}$ when the droplet was gently dropped onto the substrate.

This was not true with the other surfaces namely the $0.5 \% \mathrm{SiC} 1$-Layer, $0.1 \% \mathrm{SiC} 3 \mathrm{Layer}$ and $0.1 \% \mathrm{SiC} 1$ Layer. The film with the $0.5 \% \mathrm{SiC}, 0.125 \%$ Teflon,1-Layer configuration exhibited a contact angle of $143^{\circ}$ which when pressed made a transition to a lower energy state exhibiting a water contact angle of $118^{\circ}$. This transition to a lower energy state was also observed by Bico et al when the water droplet made a transition from $170^{\circ}$ to $140^{\circ}$. When pressed, the pressure exerted on the droplet causes the entire projected area under the droplet to be wetted due to the impalement of the droplet. In this state the apparent contact angle can be modeled with the Wenzel model. Calculation of the roughness parameter ' $r$ ' is done via AFM imaging.

The reverse trend of decreasing values of contact angles with decreasing surface area fractions can be explained by the breakdown of super-hydrophobicity. Klein et al[27] attribute the breakdown of super-hydrophobicity to two factors i) The pressure differential ' $\mathrm{P}$ ' across the meniscus being greater than a critical value $\mathrm{P}_{\text {cr }}$ and ii) contact of the curved meniscus in the asperities with the substrate. In the films with surface area fractions of $\Phi=0.092$ and $\Phi=0.036$, breakdown occurs when the pressure differential $\mathrm{P}>\mathrm{P}_{\mathrm{cr}}$, causing the droplet to spontaneously wet most of the spheres and the substrates. The second mechanism occurs when the central part of the curved meniscus touches the substrate causing a change in the meniscus shape leading to spontaneous wetting. Either 
one of this mechanism might be occurring due to the initial vibration of the droplet when the droplet is detached from the needle and rests on the substrate.

A good understanding on the spontaneous wetting of the substrate by a water droplet residing on a composite surface has been presented by Rob J. Klein et al[27].
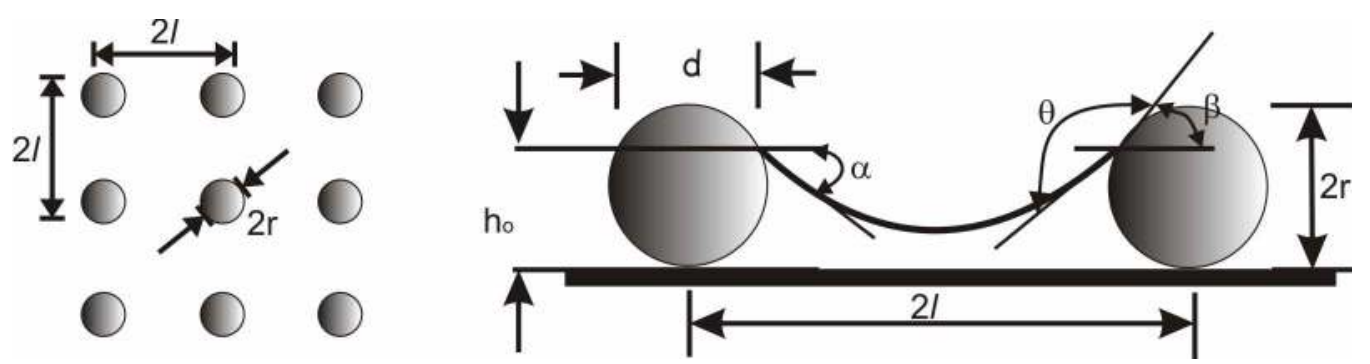

Figure 5-24 Diagram of model presented by Rob J. Klein et al[27]

They try to model a surface covered by nano-particles by considering spheres in a well ordered manner as shown in the image above.

The contact angle is denoted by $\theta$ while $\beta$ is the angle between the tangent to the particle surface at the point of contact and the horizon, $\alpha$ is the angle between the air/liquid interface and the horizon.

Now $\alpha=\theta+\beta-\pi$ and the projected area is given by $\phi=\frac{\pi r^{2}}{4 l^{2}}$ and ' $d$ ' the diameter of the wetted area of such a surface is given by

$d=2 r \operatorname{Sin} \beta$

Equation 5-xv

Using the laplace, the differential pressure across the meniscus is given by $P=-\frac{2 \phi \gamma \operatorname{Sin} \beta \operatorname{Sin}(\theta+\beta)}{r\left(1-\phi \operatorname{Sin}^{2} \beta\right)}$

Equation 5-xvi

The maximum pressure the meniscus can sustain without being forced to the substrate occurs when $\beta=\frac{\pi}{2}$. Thus the largest pressure the meniscus supports is given by $P=-\frac{2 \phi \gamma \operatorname{Cos} \theta}{r(1-\phi)}$

Equation 5-xvii 
Wetting of the substrate will occur below a critical solid-air area fraction, i.e. when the particles become too widely spaced to support the meniscus. Force exerted on a droplet of diameter $\mathrm{D}$ is given by

$F=\frac{\pi}{6} g \rho D^{3}$

Equation 5-xviii

where ' $g$ ' being the accleration due to gravity and $\rho$ being the density of the liquid. This force acts on a contact area of $\left(0.25 \pi D^{2} \operatorname{Sin}^{2} \theta^{*}\right)$ and produces a pressure on the meniscus that can be estimated to be

$P=\frac{2 g D \rho}{3 \operatorname{Sin}^{2} \theta^{*}}$

Equation 5-xix

And the citical Solid-Area fraction for spontaneous wetting is given by

$\phi_{c}=-\frac{g D \rho r}{3 \gamma \operatorname{Cos} \theta \operatorname{Sin}^{2} \theta^{*}}$

Equation 5-xx 


\subsection{Measurement of Surface Energies}

Surface energies of different surfaces viz. i) an FSM coated glass substrate ii) A glass substrate coated with an underlying layer of Silicon-Oxy-Carbide and a Teflon coating without embedded-SiC particles on top of it iii) A glass substrate coated with an underlying layer of Silicon-Oxy-Carbide and a Teflon coating with embedded-SiC particles on top of it iv) A glass substrate with a Silicon-Oxy-Carbide coating.

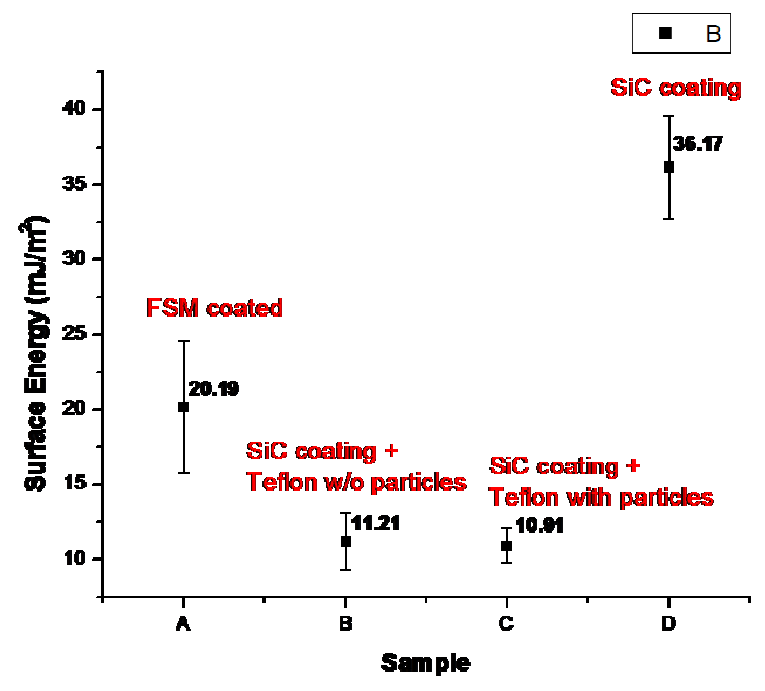

Figure 5-25 Graph of Surface energies of different surfaces

It can be seen from the above graph that the FSM coated glass substrates provide the best surface energies in comparison to the surface energies with those of Teflon. As a result better spreading and wettability is achieved when the glass substrates are FSM coated. 


\subsection{Calculation of Wenzel roughness factor ' $r$ ' and RMS Roughness}

The RMS roughness of the surfaces was calculated using AFM imaging. The Wenzel roughness factors ' $r$ ' were also calculated for each of these surfaces from the AFM data obtained.

\begin{tabular}{|c|c|c|c|c|}
\hline & $\begin{array}{c}\text { Cassie-Baxter } \\
\text { Solid Area } \\
\text { fraction ' } \Phi \text { ' }\end{array}$ & $\begin{array}{l}\text { Wenzel } \\
\text { Roughness } \\
\text { factor ' } r \text { ' }\end{array}$ & $\begin{array}{c}\text { RMS } \\
\text { Roughness }\end{array}$ & $\begin{array}{c}\text { Contact } \\
\text { Angle }\end{array}$ \\
\hline $\begin{array}{l}0.1 \%(w t \%) \mathrm{SiC} \\
\text { embedded in } \\
0.125 \%(\mathrm{wt} \%) \text { Teflon: } \\
\text { Single Layer } \\
\text { configuration }\end{array}$ & $0.036 \pm .006$ & 1.09 & $114 \pm 6 \mathrm{~nm}$ & $120^{\circ}$ \\
\hline $\begin{array}{l}0.1 \%(\mathrm{wt} \%) \mathrm{SiC} \\
\text { embedded in } \\
0.125 \%(\mathrm{wt} \%) \text { Teflon: } \\
\text { triple Layer } \\
\text { configuration }\end{array}$ & $0.092 \pm .01$ & 1.12 & $130 \pm 4 \mathrm{~nm}$ & $128^{\circ}$ \\
\hline $\begin{array}{l}0.5 \%(w t \%) \mathrm{SiC} \\
\text { embedded in } \\
0.125 \%(\mathrm{wt} \%) \text { Teflon: } \\
\text { Single Layer } \\
\text { configuration }\end{array}$ & $0.17 \pm .01$ & 1.19 & $143 \pm 4 \mathrm{~nm}$ & $143^{\circ}$ \\
\hline $\begin{array}{l}0.5 \%(w t \%) \mathrm{SiC} \\
\text { embedded in } \\
0.125 \%(\mathrm{wt} \%) \text { Teflon: } \\
\text { triple Layer } \\
\text { configuration }\end{array}$ & $0.316 \pm .05$ & 1.38 & $159 \pm 5 \mathrm{~nm}$ & $156^{\circ *}$ \\
\hline $\begin{array}{l}0.5 \%(\mathrm{wt} \%) \mathrm{SiC} \\
\text { embedded in } \\
0.125 \%(\mathrm{wt} \%) \text { Teflon: } \\
10 \text { Layer configuration }\end{array}$ & $.872 \pm .048$ & 1.61 & $195 \pm 2 \mathrm{~nm}$ & $<160^{\circ}$ \\
\hline
\end{tabular}

Table 5-29: CB Solid Area fraction ' $\Phi$ ', Wenzel Roughness factor ' $r$ ' and RMS Roughness calculated using SEM and AFM imaging.

\footnotetext{
* water droplet was deposited using a non-negligible pressure for measurement of contact angle.
} 
5.20.1 0.1\%(wt\%) SiC nano-particles embedded in $0.125 \%(w t \%)$ Teflon: Single Layer configuration
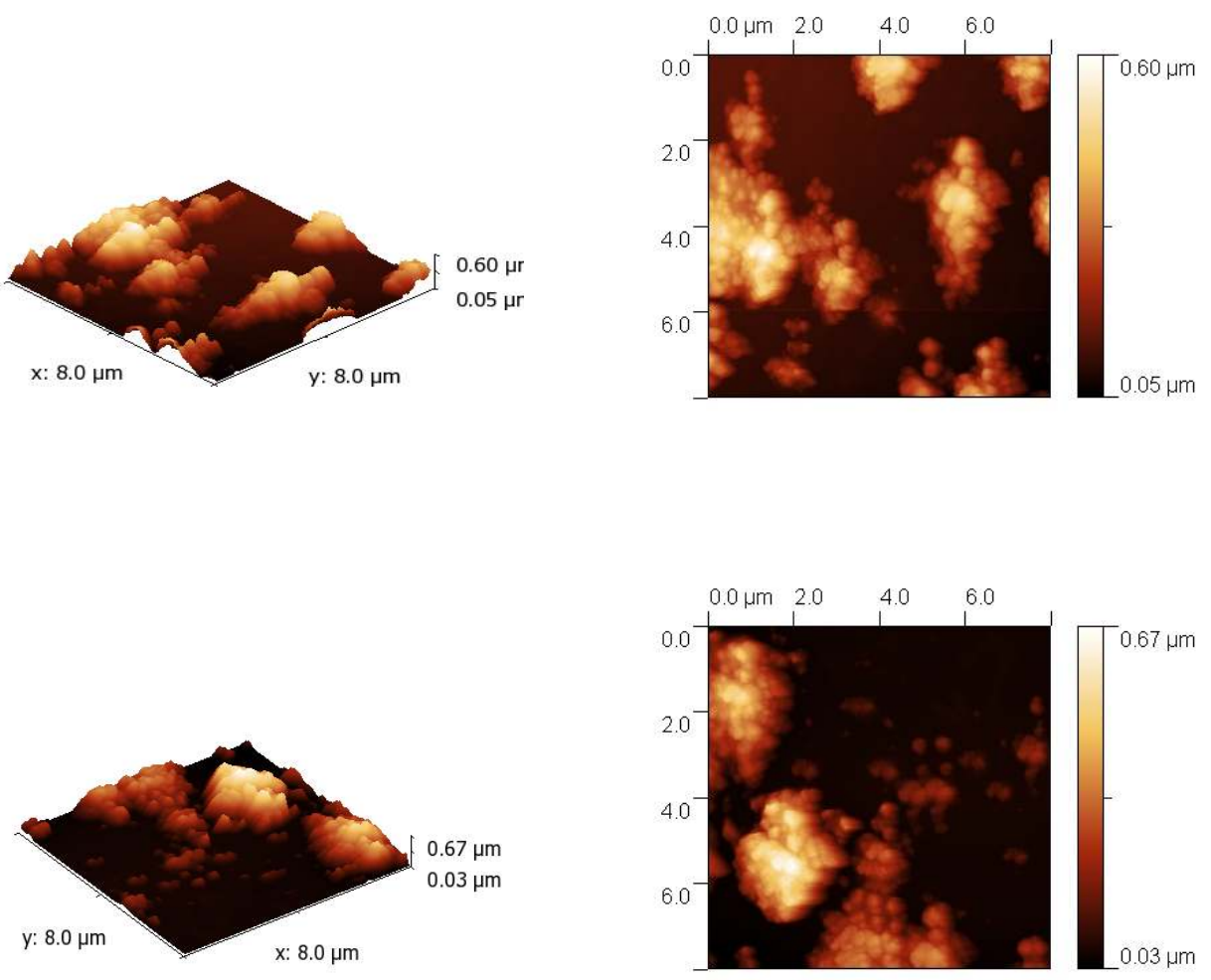

\section{RMS Roughness: $114 \pm 6 n m$}

Table 5-30: AFM images of the $0.1 \%(w t \%) \mathrm{SiC}$ in $0.125 \%(w t \%)$ Teflon coated Surface(single Layered)

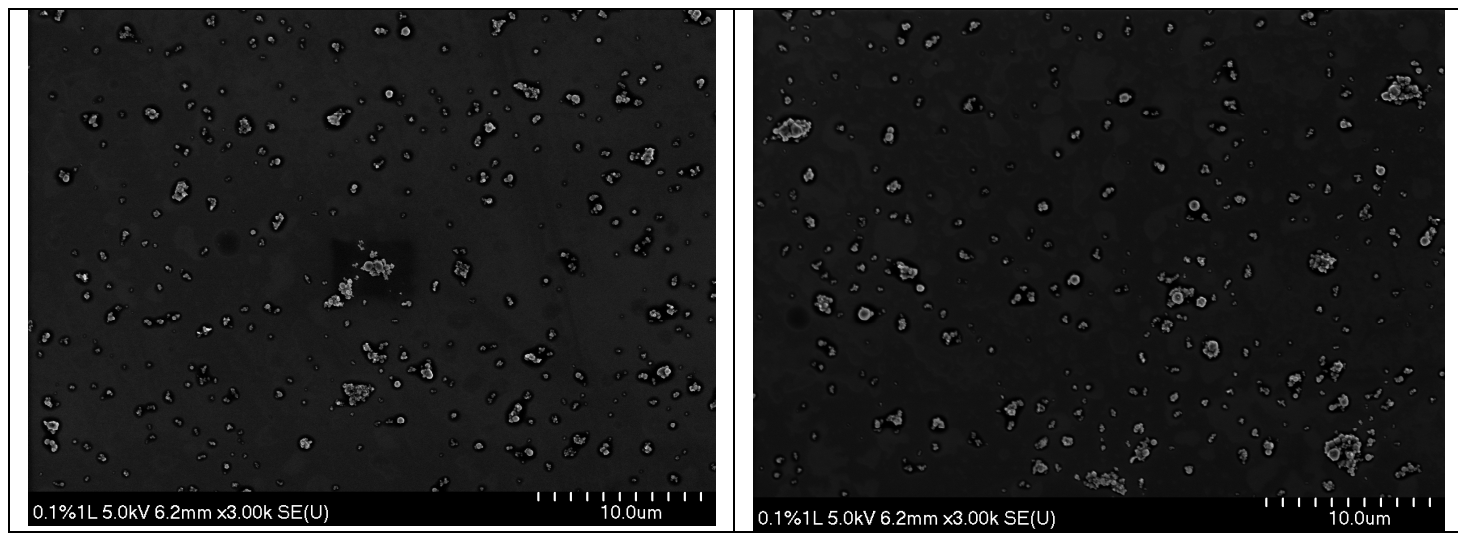

Table 5-31: SEM of 0.5\%(wt\%) SiC in $0.125 \%(w t \%)$ Teflon coated Surface(single layer) 
5.20.2 0.1\%(wt\%) SiC nano-particles embedded in 0.125\%(wt\%) Teflon: 3 Layer configuration
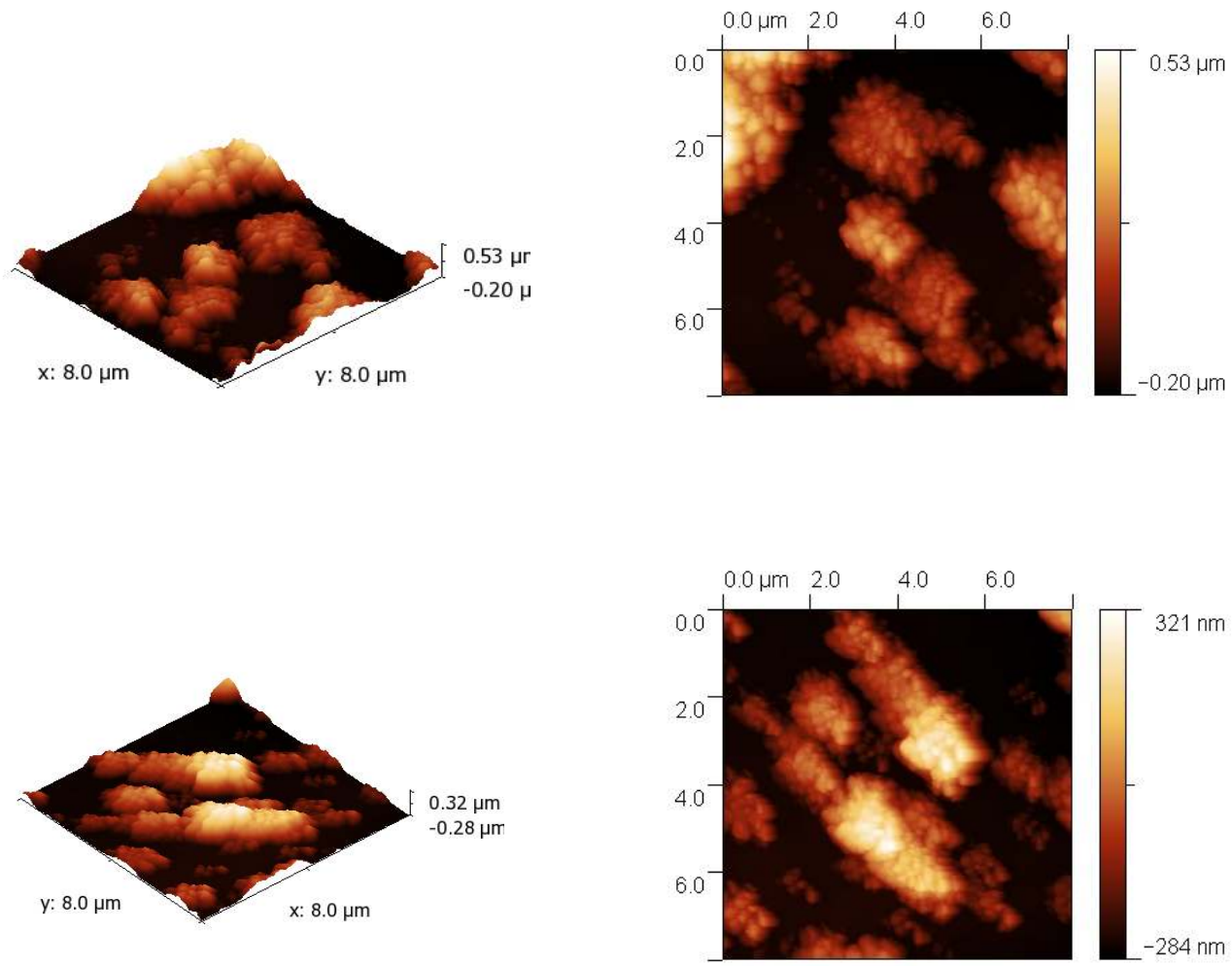

\section{RMS Roughness: $130 \pm 4 \mathrm{~nm}$}

Table 5-32: AFM images of the $0.1 \%(w t \%)$ SiC in $0.125 \%(w t \%)$ Teflon coated Surface(triple layered)
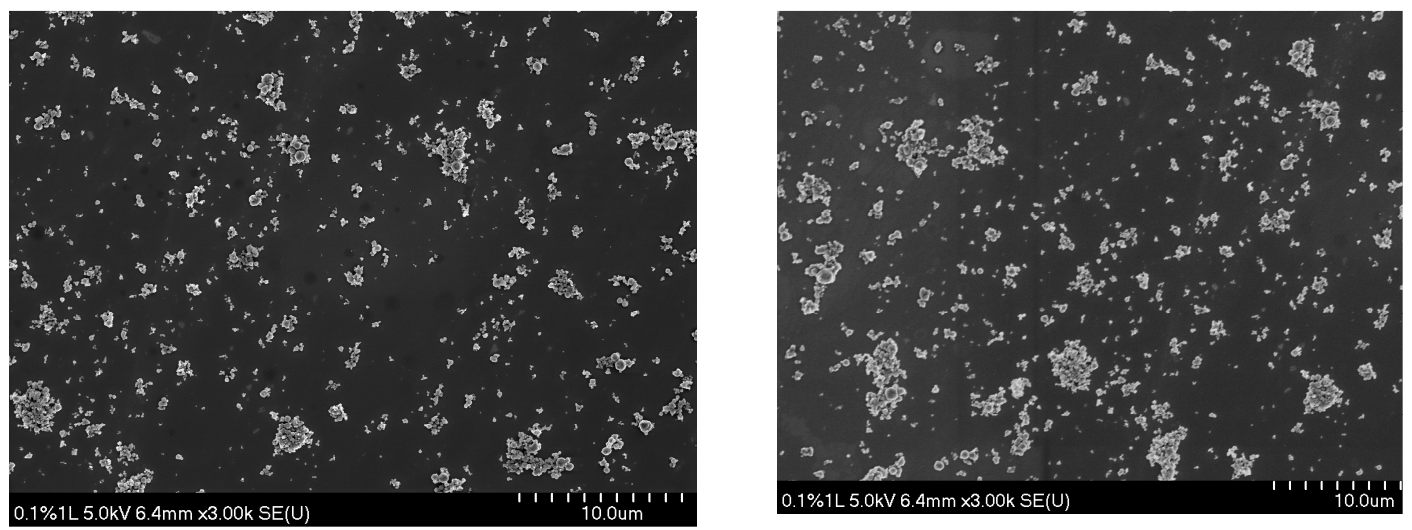

Table 5-33: SEM of $0.1 \%(w t \%) ~ S i C$ in $0.125 \%(w t \%)$ Teflon surface(triple layered) 
$5.20 .30 .5 \%(w t \%)$ SiC nano-particles embedded in $0.125 \%(w t \%)$ Teflon: Single Layer configuration
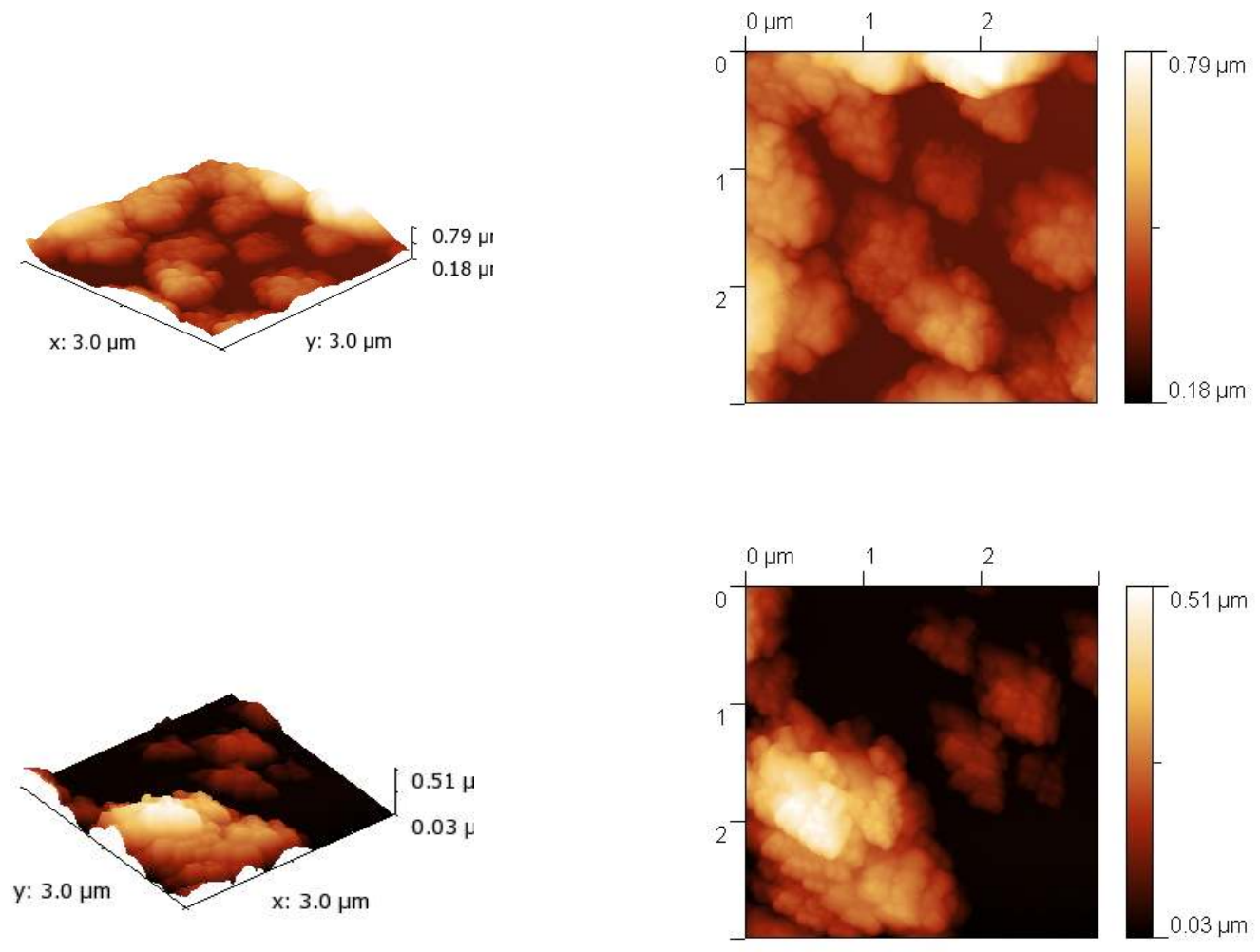

\section{RMS Roughness: $143 \pm 4 n m$}

Table 5-34: AFM images of the $0.5 \%(w t \%)$ SiC in $0.125 \%(w t \%)$ Teflon coated Surface(Single Layered)
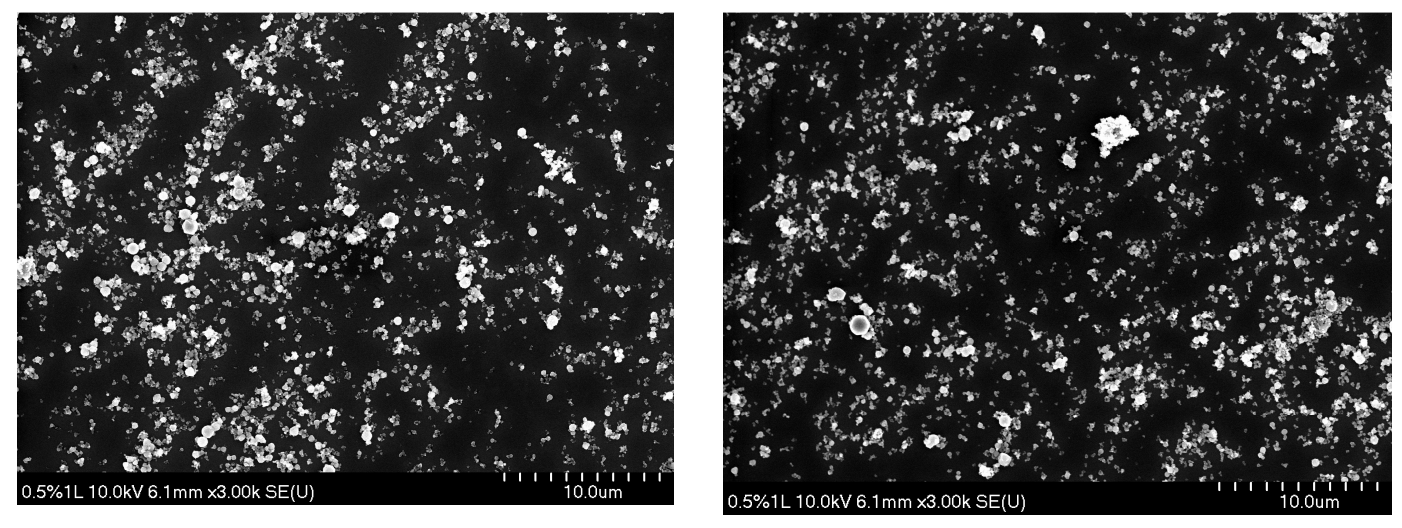

Table 5-35: SEM of $0.5 \%(w t \%) ~ S i C$ in $0.125 \%(w t \%)$ Teflon surface(single layered) 
5.20.4 0.5\%(wt\%) SiC nano-particles embedded in $0.125 \%(w t \%)$ Teflon: 3 Layer configuration
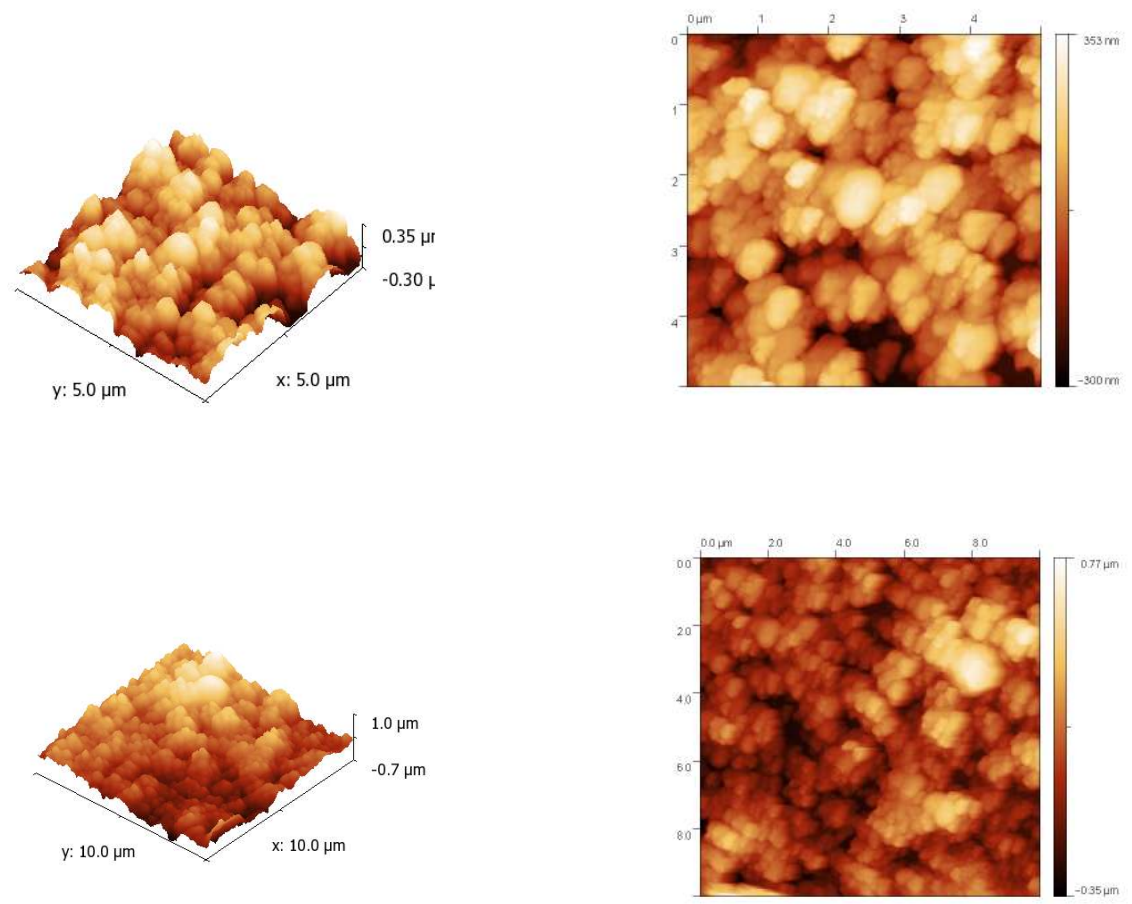

RMS Roughness: $159 \pm 5 \mathrm{~nm}$

Table 5-36: AFM images of $0.5 \%(w t \%)$ SiC in $0.125 \%(w t \%)$ Teflon coated Surface(triple layered)
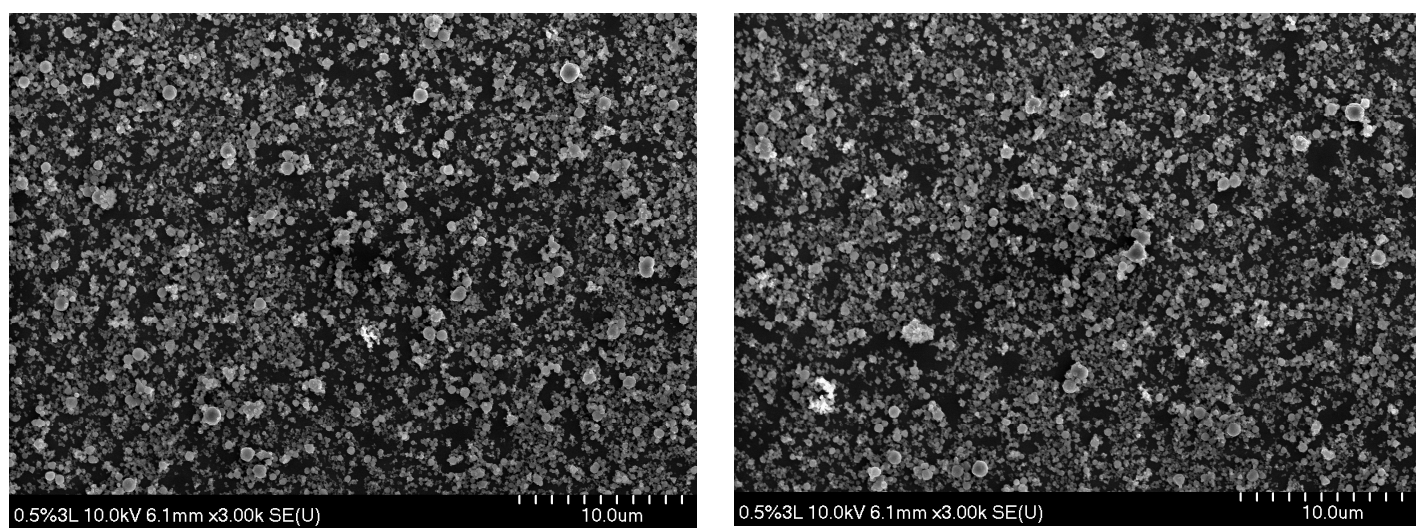

Table 5-37: SEM of $0.5 \%(w t \%)$ SiC in $0.125 \%(w t \%)$ Teflon surface(triple layered) 
$5.20 .50 .5 \%(w t \%)$ SiC nano-particles embedded in $0.125 \%(w t \%)$ Teflon: 10 Layer configuration
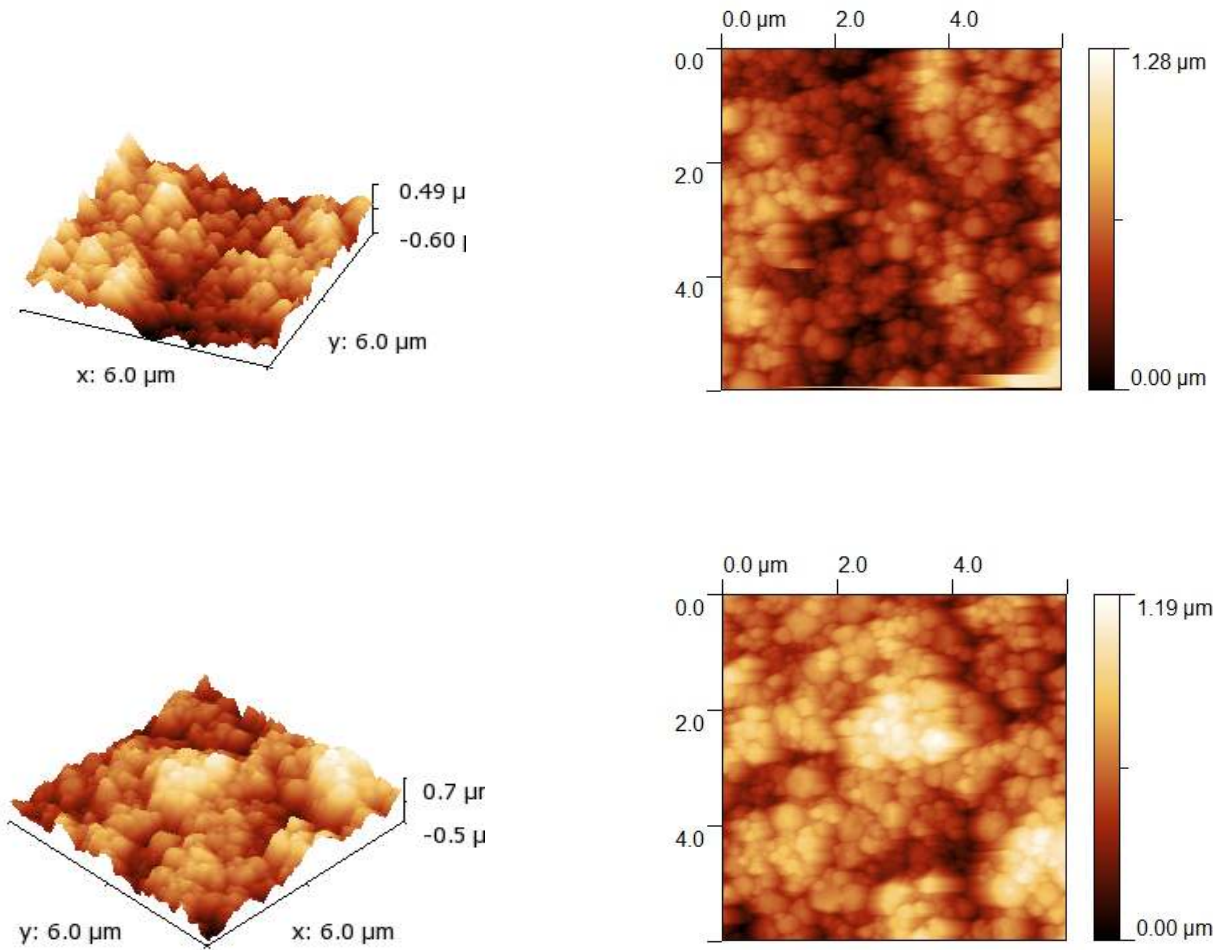

\section{RMS Roughness: 195 $\pm 2 \mathrm{~nm}$}

Table 5-38: AFM images of the $0.5 \%(w t \%)$ SiC in $0.125 \%(w t \%)$ Teflon coated Surface(10 Layered)

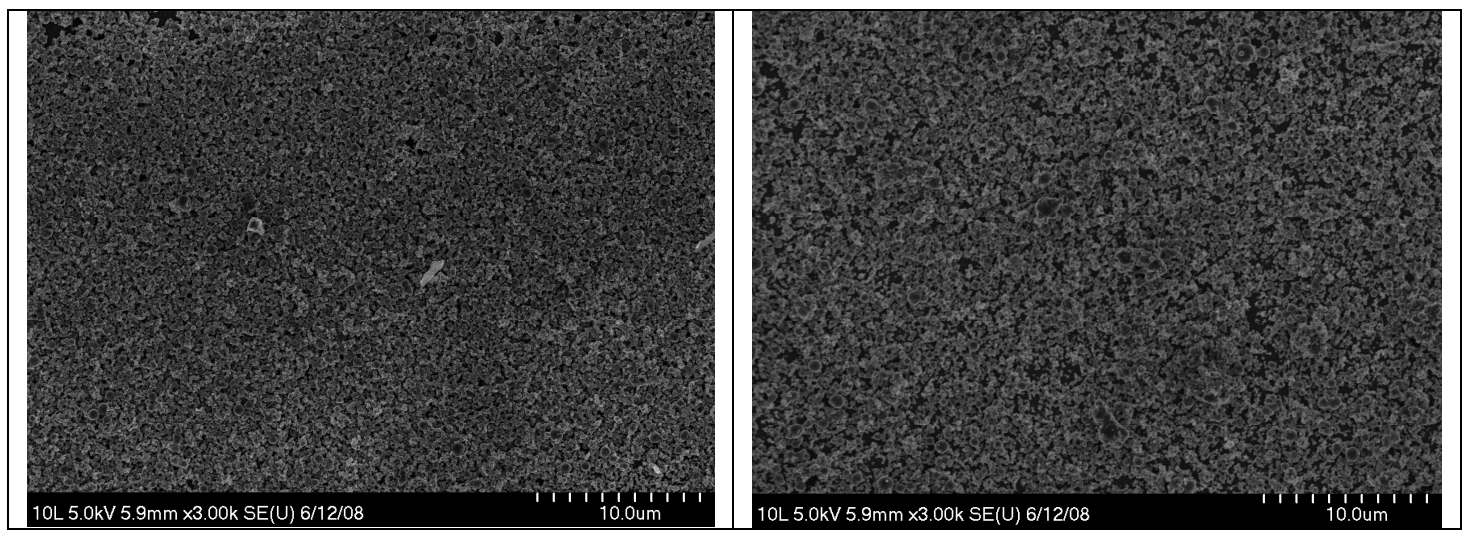

Table 5-39: SEM of $0.5 \%(w t \%)$ SiC in $0.125 \%(w t \%)$ Teflon(10 Layered) surface 


\subsection{Discussion on RMS roughness}

As can be seen in table 5-29, the RMS roughness of all the surfaces with different solidair area fractions were found using AFM imaging. The Wenzel and the Cassie-Baxter equations do not consider the RMS roughness while predicting the apparent contact angles on rough surfaces.

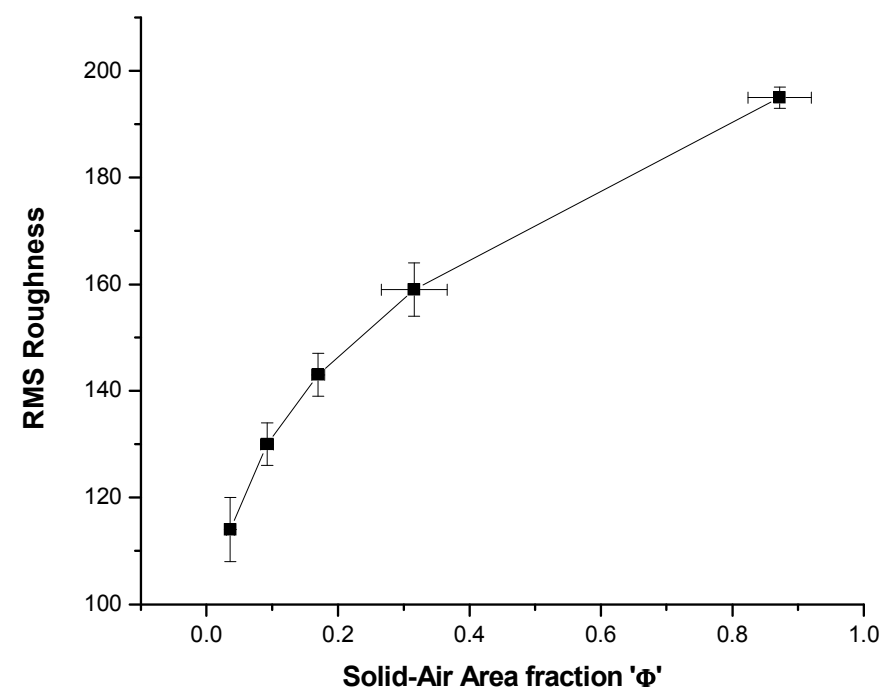

Figure 5-26 Graph of RMS Roughness v/s Solid-Air Area fraction ' $\Phi$ '

As can be seen from the data, there seems to be a linear relationship between the RMS roughness and the contact angles for surfaces with solid-area fractions ' $\Phi$ ' from 0.036 to 0.872; The contact angles appear to increase with increasing roughness as seen from the RMS roughness data.

On close examination of the RMS roughness formula, $S_{q}$

$S_{q}=\sqrt{\frac{1}{M N} \sum_{k=0}^{M-1} \sum_{l=0}^{N-1}\left[z\left(x_{k}, y_{l}\right)-\mu\right]^{2}}$ Equation 5-xxi 
where $\mu$ is the mean value of the height, $z$, across all in-plane coordinates $(x, y)$ :

$\mu=\frac{1}{M N} \sum_{k=0}^{M-1} \sum_{l=0}^{N-1} z\left(x_{k}, y_{l}\right)$

\section{Equation 5-xxii}

The RMS roughness has some inherent limitations[68] that are often neglected. Reporting the RMS roughness is almost always useful, but frequently inadequate in accurately describing surface topography in a meaningful quantitative way. The limitations can be summarized by saying that RMS roughness measurement can give nearly or identically the same numerical result for two surfaces whose roughness are qualitatively different, or very different; sometimes so different that even a simple visual inspection of the AFM images will reveal.

One important reason RMS roughness is sometimes inadequate[68] is that it is computed indiscriminately towards the polarity of the height value at a given pixel, relative to the mean height value across all the pixels in the image. In other words, as the formula shows, height values smaller than and larger than the mean value end up contributing to the RMS roughness the same way, i.e., as positive numbers. The result is that the RMS roughness may measure (very) nearly the same for two different surfaces, one for example a flat surface with many holes, the other a flat surface with many peaks.

In order to distinguish between these two kinds of surfaces, a different kind of parameter from RMS roughness is required which is called skewness[68] and it is not nearly as widely used as the RMS roughness is. The skewness, $S_{s k}$, is defined in a way that can quantitatively describe the asymmetry of a height distribution about the mean 
$S_{s k}=\frac{1}{M N S_{q}^{3}} \sum_{k=0}^{M-1} \sum_{l=0}^{N-1}\left[z\left(x_{k}, y_{l}\right)-\mu\right]^{3}$

\section{Equation 5-xxiii}

The formula is similar to the one for RMS, but unlike RMS (roughness), the skewness can take on positive and negative values as well as zero (even if the surface is not perfectly smooth), because each term in the double summation is raised to an odd power.
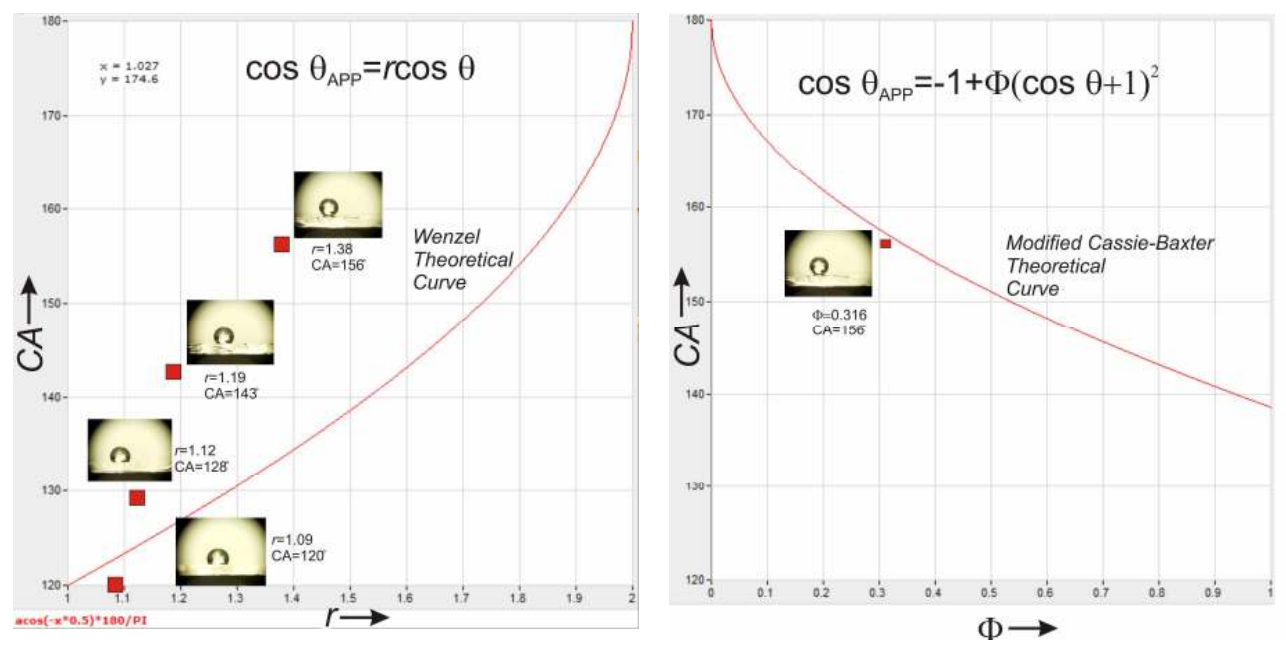

Figure 5-27 Graph of the Wenzel and modified Cassie-Baxter theoretical curves with obtained experimental data

The above graphs show the theoretical curves using the Wenzel and the modified CassieBaxter models. As can be seen the contact angles with water keep increasing on surfaces with increasing Wenzel roughness ratios ' $r$ '. The data point at $r=1.38$ results in a contact angle of $156^{\circ}$. Since the droplet on this surface was deposited by applying a nonnegligible pressure, it is believed that the droplet could be forced to make a transition from the cassie to the wenzel state and hence it was plotted with the wenzel formula. This data point was also plotted with the Modified Cassie-Baxter exquation and a good fit was seen with the theoretical curve.

At first thought one would expect that the surfaces with wenzel roughness ratios 1.09, 1.12 and 1.19 having solid-area fractions of $.036, .092, .17$ to have very high contact 
angles, as the apparent contact angles resulting from these surfaces were $172^{\circ}, 167^{\circ}, 163^{\circ}$ respectively using the modified Cassie-Baxter equation. Spontanoeus wetting of the surfaces causes the droplet to transition and wet the surfaces resulting in wenzel state contact angles. An explanation and derivation on the critical solid-air area fraction below which spontaneous wetting takes place has been presented by Rob J. Klein which has been included in this thesis. 


\section{CHAPTER 6: CONCLUSIONS}

\subsection{Results Summary}

At the beginning of this thesis, the question as to whether a facile route in producing super-hydrophobic surfaces was very intriguing. Other questions as to whether such a surface could be processed at a low temperature, the abrasion/scratch resistance of such a coating and the transparency of the developed coating were taken into consideration before choosing the routes that were implemented in developing this surface. A sol-gel route was chosen as the first approach as sol-gel based films possess excellent virtues such good mechanical strengths, transparencies above $90 \%$ in the visible etc. Roughness in our sol-gel films was induced through porosity with the help of templating agents. Roughness could also be induced in these sol-gel based films via the incorporation of nano-particles in the precursor solutions as demonstrated in the Teflon films. Elimination of templating agents in a low temperature route is a challenge as in high temperature processes, the organic components disintegrate.

Incorporation of nano-particles such as Silica, silicon-nitride, Silicon carbide etc with the desired size into these sol-gel films could help in creating the desired roughness. The restricted time within which a low temperature process could be developed led us to explore alternate routes.

The porous Teflon films using micro-emulsions did not help in enhancing the contact angles as the desired roughness of the pores could not be controlled. Study on the pore size by varying different parameters could not be carried out because of time constraints. 
Teflon polymeric films which could be rendered super-hydrophobic with the help of added nano-particles to induce roughness were the best approach.

A study on the variation in RMS roughness with different area coverage of nano-particles helped us in understanding how an enhancement in the contact angles is achieved.

These films possessed excellent super-hydrophobic characteristics but suffered from low mechanical strengths.

\subsection{Future work}

The low temperature sol-gel route would be the most ideal route in the development of such a super-hydrophobic surface. A lot of literature already exists on the development of sol-gel based super-hydrophobic films but none of them speak about the low-temperature processing of such films.

The hardness of the Teflon films embedded with the SiC nano-particles could be increased if a plasma deposited $\mathrm{SiC}$ or a fluorinated-Silicon-Oxy carbide layer could be deposited on such a rough surface. The thickness of this coating would have to be no less than half the minimum feature size of the surface to preserve the original morphology of the surface. In this way, the films would posses super-hydrophobic as well as mechanical strengths. Another way in which better adhesion of such a super-hydrophobic surface could be created would be by spin coating such a Teflon/ $\mathrm{SiC}$ solution on a cured smooth Teflon film. This approach was followed but did not result in smooth coatings as the nano-particles were seen to cluster at different places on the film. This was due to the underlying Teflon films not being perfectly smooth as seen by the optical images of pure Teflon films. 
Another thing that would help in improving the transparencies of these films are the incorporation of smaller SiC particles of the order of 10-30nm. Scattering of light could be drastically reduced due to smaller particle sizes.

Using sacrificial colloidal nano-particles along with $\mathrm{SiC}$ nanoparticles could help in inducing roughness in $2 \%$ Teflon films which would leave the films having a porous like structure after the sacrifical nano-particles disintegrate during the curing process. 


\section{References}

1 Otten, A. Herminghaus, S. "How Plants Keep Dry: A Physicist's Point of View" Langmuir 20, (2004): 2405-2408.

2 Erbil, H. Y., Demirel, A. L., Avci, Y. \& Mert, O. "Transformation of a Simple Plastic into a Superhydrophobic Surface” Science 299, (2003): 1377-1380.

3 Nakajima, A., Hashimoto, K. \& Watanabe, T. "Recent Studies on Super-hydrophobic Films" Monatshefte für Chemie 132, (2001): 31-41.

4 Blossey, R. "Self-cleaning surfaces-virtual realities" Nature Materials 2, (2003): 301306.

5 E. A. Baker, Linnean Society Symposium Series, ed. D. F. Cutler, K. L. Alvin and C. E. Price, "Chemistry and Morphology of Plant Epicuticular Waxes, in The Plant Cuticle" Academic Press, London, 1982.

6 Jeffree, C. (1986) The cuticle, epicuticular waxes and trichomes of plants, with reference to their structure, functions and evolution In: Juniper, B., Southwood, R. eds. , Insects and the Plant Surface, E. Arnold, London, pp $23-135$

7 T. L. Sun, L. Feng, X. F. Gao and L. Jiang, "Artificial lotus leaf structures from assembling carbon nanotubes and their applications in hydrophobic textiles" J. Mater. Chem. 17, (2007): 1071 - 1078

8 Y T Cheng1, D E Rodak2,CAWong3 and C A Hayden "Effects of micro- and nanostructures on the self-cleaning behaviour of lotus leaves" Y T Cheng et al Nanotechnology 17, (2006): 1359-1362

9 Acatay,Kazim "Generation of superhydrophobic surfaces by Electrospinning process" Phd diss., Sabancı University 2004.

10 Barthlott, W. \& Neinhuis, C. "Purity of the sacred lotus, or escape from contamination in biological surfaces" Planta 202, (1997): 1-8

11 Joong Tark Han, Xurong Xu, ilwon Cho "Diverse Access to Artificial Superhydrophobic Surfaces Using Block Copolymers” Langmuir, 21 (15) (2005): $6662-6665$

12 M. Hikita, K. Tanaka, T. Nakamura, T. Kajiyama and A. Takahara "Construction of superhydrophobic surfaces by fibrous aggregation of perfluoroalkyl chaincontaining organogelators", Langmuir 21, (2005): 7299. 
13 M. H. Sun, C. X. Luo, L. P. Xu, H. Ji, O. Y. Qi, D. P. Yu and Y. Chen "Artificial lotus leaf by nanocasting", Langmuir 21, (2005): 8978

14 B. He, N. A. Patankar and J. Lee, "On the modeling of Super-hydrophobic surfaces" Langmuir 19, (2003): 4999

15 J. Fresnais, L. Benyahia and F. Poncin-Epaillard, "Dynamic (de)wetting properties of superhydrophobic plasma-treated polyethylene surfaces" Surface and Interface Analysis, 38, (2006): 144

16 S. Minko, M. Muller, M. Motornov, M. Nitschke, K. Grundke and M. Stamm, J. Am "Low pressure plasma-based approaches to fluorocarbon polymer surface modification". Chem. Soc. 125, (2003): 3896

17 R. Furstner, W. Barthlott, C. Neinhuis and P. Walzel, "Wetting and self-clening properties of artificial super-hydrophobic surfaces" Langmuir 21, (2005): 956

18 E. Martines, K. Seunarine, H. Morgan, N. Gadegaard,C. D. W. Wilkinson and M. O. Riehle, "Superhydrophobicity and Superhydrophilicity of Regular Nanopatterns" Nano Lett. 5, (2005): 2097-2103

19 H. Yıldırım Erbil, A. Levent Demirel, Yonca Avc1, Olcay Mert "Transformation of a Simple Plastic into a Superhydrophobic Surface" Science 28, (February 2003): Vol. 299. no. 5611

20 Xue-Mei Li, David Reinhoudt and Mercedes Crego-Calama. "What do we need for a superhydrophobic surface? A review on the recent progress in the preparation of superhydrophobic surfaces" Chem. Soc. Rev 36, (2007): $1350-1368$

21 Yang-Tse Cheng. "Is the lotus leaf superhydrophobic?” Appl. Phys. Lett. 86, (2005): 144101

22 Xue-Mei Li, David Reinhoudt and Mercedes Crego-Calama. "What do we need for a superhydrophobic surface? A review on the recent progress in the preparation of superhydrophobic surfaces" Chem. Soc. Rev 36, (2007): $1350-1368$

23 M. H. Sun, C. X. Luo, L. P. Xu, H. Ji, O. Y. Qi, D. P. Yu and Y. Chen "Artificial lotus leaf by nanocasting", Langmuir 21, (2005): 8978

24 J. Fresnais, L. Benyahia and F. Poncin-Epaillard, "Dynamic (de)wetting properties of super-hydrophobic plasma-treated polyethylene surfaces" Surf. Interface Anal. 38, (2006): 144 
25 S. Minko, M. Muller, M. Motornov, M. Nitschke, K. Grundke and M. Stamm, "Low pressure plasma-based approaches to fluorocarbon polymer surface modification" J. Am. Chem. Soc. 125, (2003): 3896

26 J. Y. Shiu, C. W. Kuo, P. L. Chen and C. Y. Mou, "Fabrication of Tunable Superhydrophobic Surfaces by Nanosphere Lithography" Chem. Mater. 16, (2004): 561.

27 Rob J. Klein, P. Maarten Biesheuvel "Producing Super-hydrophobic surfaces with Nano-Silica Spheres" Unpublished,

28 L. Zhai, F. C. Cebeci, R. E. Cohen and M. F. Rubner "Stable superhydrophobic coatings from polyelectrolyte multilayers", Nano Lett. 4, (2004): 1349.

29 J. T. Han, Y. Zheng, J. H. Cho, X. Xu and K. Cho, "Stable superhydrophobic organicinorganic hybrid films by electrostatic self-assembly" J. Phys.Chem. B, (2005): 109, 20773.

30 Shang, H.M., Wang, Y., Limmer, S. J., Chou, T. P., Takahashi, K. and Cao, G. Z., "Optically Transparent Superhydrophobic Silica-Based Films" Thin Solid Films 37, (2005): 472

31 Hikita, M., Tanaka, K., Nakamura,T., Kajiyama, T., and Takahara, A. "Construction of superhydrophobic surfaces by fibrous aggregation of perfluoroalkyl chain-containing organogelators", Langmuir 21, (2005): 7299.

32 Burkarter, Ezequiel, K Saul, Cyro, Thomazi, Fabiano, C Cruz, Nilson, M Zanata, Silvio, Lucimara S Roman and H Schreiner, Wido "Electrosprayed superhydrophobic PTFE: a non-contaminating surface" J. Phys. D: Appl. Phys. 40 (2007): 7778-7781

33 L. Feng, Z. Zhang, Z. Mai, Y. Ma, B. Liu, L. Jiang and D. Zhu, "A SuperHydrophobic and Super-Oleophilic Coating Mesh Film for the Separation of Oil and Water" Angewandte Chemie International Edition 43, (November 2003): 2012 - 2014

34 Xiu, Yonghao, Zhu, Lingbo, W. Hess, Dennis.; Wong, C. P. "Superhydrophobic Silica Thin Films Prepare by Sol-Gel Process for Antistiction of MEMS Devices" IEEE - High Density packaging and Microsystem Integration, 2007. HDP '07. International Symposium 
35 Chang, Kuei-Chien, Chen, Yu-Kai, Hui Chen "Fabrication of superhydrophobic silicabased surfaces with high transmittance by using polypropylene and tetraeyhoxysilane precursors" Journal of Applied Polymer Science 107, Issue 3, (October 2007): 1530 - 1538

36 Erbil, H. Y., Demirel, A. L., Avci, Y. \& Mert, O. "Transformation of a Simple Plastic into a Superhydrophobic Surface" Science 299, (2003): 1377-1380.

37 Xue-Mei Li, David Reinhoudt and Mercedes Crego-Calama. "What do we need for a superhydrophobic surface? A review on the recent progress in the preparation of superhydrophobic surfaces" Chem. Soc. Rev 36, (2007): $1350-1368$

38 Bhushan, Bharat and Jung, Yong Chae "Wetting, adhesion and friction of superhydrophobic and hydrophilic leaves and fabricated micro/nanopatterned surfaces" J. Phys.: Condens. Matter 20, (2008): 225010

39 Bico, J., Marzolin, C., and Quere, D. "Pearl drops" Europhys. Lett. 47 (2), (1999): $220-226$

40 Wenzel, R. N. "Resistance of Solid Surfaces to Wetting by Water", Industrial and Engineering Chemistry 28, (1936): 988-994.

41 Cassie, A. B. D., "Contact Angles" Discussions of the Faraday Society 3, (1948): 1115

42 Barbieri, Laura "Wetting properties of flat-top periodically structured superhydrophobic surfaces", PhD diss., Università degli Studi di Genova, Italie

43 Cassie, A. B. D.; Baxter, S. "Wettability of Porous Surfaces", Transactions of the Faraday Society 40, (1944): 546-551

44 Frédéric Michel "Surface free energy evaluation of building materials" University of Liege (ULg), B5, Liège 4000, Belgium. Poster at E-MRS Fall Meeting 2005, Symposium G, by Courard Luc

45 Michel A. Aegerter, Martin Mennig Sol-Gel Technologies for glass producers and users Springer; 1 edition (October 29, 2004)

46 Brinker, C. Jeffrey/ Scherer, George Sol-Gel Science W. / HARDCOVER / 1990

47 Tao, Cheng, Huang, Jian Guo, Jun Bai "Noodle-like Mesoporous Silica Templated by Polyelectrolyte/Surfactant Complex" Chinese Chemical Letters 14, No. 11, (2003): $1175-1178$ 
48 Dow Corning, A guide to Silane Solutions.

http://www.dowcorning.com/content/publishedlit/SILANE-GUIDE.pdf, Accessed July 16, 2008

49 Yildirim, Ismail "Determination of surface free energies of talc from contact angles measured on flat and powdered samples" PhD diss., Virginia Polytechnic Institute and State University, Blacksburg, Virginia April 2001

50 de Meijer, Mari, Haemers,Sander, Cobben, Wiro and Militz, Holger "Surface Energy Determinations of Wood: Comparison of Methods and Wood Species" Langmuir 16, (2000): 9352-9359

51 Lu, Yunfeng; Ganguli, Rahul; A. Drewien, Celeste; Anderson, Mark T.; Brinker, C. Jeffrey; Gong, Weilang; Guo, Yongxing; Soyez, Hermes; Dunn, Bruce; Michael H. Huang and Jeffrey I. Zink "Continuous formation of supported cubic and hexagonal mesoporous films by sol-gel dip-coating" Letters to Nature; Nature 389, (July 1997): 364-368

52 Korampally, Venumadhav, Gangopadhyay, Shubhra "Rapid formation of Ultra low refractive index nanoporous dielectric films based on PMSSQ nanoparticles"

53 S. Bok "Electrochemical Properties of Carbon Nanoparticles Entrapped in Silica Matrix"

54 H. Y1ldırım Erbil, A. Levent Demirel, Yonca Avc1, Olcay Mert "Transformation of a Simple Plastic into a Superhydrophobic Surface" Science 28, (February 2003): Vol. 299. no. 5611

55 P Manoudis, S Papadopoulou, I Karapanagiotis, A Tsakalof, I Zuburtikudis and C Panayiotou "Polymer-Silica nanoparticles composite films as protective coatings for stone-based monuments" - Journal of Physics: Conference Series 61 (2007): 1361-1365

56 del Monte, Francisco and Levy, David "Spectroscopy and optical properties of HITCdoped silica sol-gel glasses Optical Materials", J. Optical materials 13, Issue 1, (October 1999): 17-25

57 A. T. Cho, T. G. Tsai,b C. M. Yang,a K. J. Chao, and F. M. Pan. "Plasma Treatments of Molecularly Templated Nanoporous Silica Films" Electrochem. SolidState Lett. 4, Issue 4, (April 2001): G35-G38

58 Haibin Li, Toyoki Kunitake "Efficient proton conduction of nanometer thick film of porous silica as prepared by Oxygen Plasma treatment" Microporous and mesoporous materials 97, (2006): 42-48 
59 A. Patankar, Neelesh "On the Modeling of Hydrophobic Contact Angles on Rough Surfaces" Langmuir 19 (4) (Feb 2003): 1249-1253

60 Pieter Van der Wal \& Ullrich Steiner. "Super-hydrophobic surfaces made from Teflon", Soft Matter 3, (2007): 426 - 429

61 J. Bico, C. Marzolin, D. Quere. "Pearl Drops” Europhysics Letters 47, (1999): 220

62 Rob J. Klein, P. Maarten Biesheuvel, Ben C. Yu, Carl D. Meinhart, and Fred F. Lange "Producing Super-hydrophobic surfaces with Nano-Silica Spheres" Journal of the American Ceramic Society 89 Issue 3, (March 2006): 817822

63 Nakayama, Norio; Hayashi, Toyoharu "Preparation and characterization of TiO2 and Polymer Nano-composite Films with high refractive Index" Journal of Applied Polymer Science 105, Issue 6, (June 2007): 3662 - 3672

64 Luo, Ying; Rong, Min Zhi; Zhang, Ming Qiu "Tribological behavior of epoxy composites containing reactive $\mathrm{SiC}$ nanoparticles" Journal of Applied Polymer Science 104, Issue 4, (Feb 2007): 2608 - 2619

65 Chou, Chih-Wei; Hsu, Shan-hui; Chang, Han; Sheng-Mao Tseng c; Hong-Ru Lin "Enhanced thermal and mechanical properties and biostability of polyurethane containing silver nanoparticles" Polymer Degradation and Stability 91, (2006): 1017-1024

66 Jia, Q.L.; Zhang, M.Q.; Rong, M.Z.; B. Wetzel and K. Friedrich "Friction and wear of epoxy composites containing surface modified SiC nanoparticles" Tribology Letters 20, 2, (October 2005): 115-123(9)

67 Erbil, H. Y., Demirel, A. L., Avci, Y. \& Mert, O. “Transformation of a Simple Plastic into a Superhydrophobic Surface” Science 299, (2003): 1377-1380.

68 Horwitz, Joan "RMS roughness: the measurement that may sometimes be skewed" Nano Measure; Measurements in Nanotechnology Blog http://nano.tm.agilent.com/blog/2008/02/04/rms-roughness-themeasurement-that-may-sometimes-be-skewed/ Accessed: July 16, 2008 